\title{
Design and development of an Energy Efficiency Knowledge Center (EEKC)
}

Seyed Mohammad Jalali

West Virginia University

Follow this and additional works at: https://researchrepository.wvu.edu/etd

\section{Recommended Citation}

Jalali, Seyed Mohammad, "Design and development of an Energy Efficiency Knowledge Center (EEKC)" (2011). Graduate Theses, Dissertations, and Problem Reports. 4734.

https://researchrepository.wvu.edu/etd/4734

This Thesis is protected by copyright and/or related rights. It has been brought to you by the The Research Repository @ WVU with permission from the rights-holder(s). You are free to use this Thesis in any way that is permitted by the copyright and related rights legislation that applies to your use. For other uses you must obtain permission from the rights-holder(s) directly, unless additional rights are indicated by a Creative Commons license in the record and/ or on the work itself. This Thesis has been accepted for inclusion in WVU Graduate Theses, Dissertations, and Problem Reports collection by an authorized administrator of The Research Repository @ WVU. For more information, please contact researchrepository@mail.wvu.edu. 


\title{
DESIGN AND DEVELOPMENT OF AN ENERGY EFFICIENCY KNOWLEDGE CENTER (EEKC)
}

By

Seyed Mohammad Jalali

Thesis submitted to the College of Engineering and Mineral Resources at West Virginia University in partial fulfillment of the requirements

for the degree of

\author{
Master of Science \\ in \\ Industrial Engineering
}

Approved by

Bhaskaran Gopalakrishnan, Ph.D., Committee Chairperson Robert C. Creese, Ph.D.

Shahab Mohaghegh, Ph.D.

Department of Industrial and Management Systems Engineering

\author{
Morgantown, West Virginia \\ 2011
}

Keywords: Energy Efficiency Assessment, Knowledge Center 


\section{Abstract \\ DESIGN AND DEVELOPMENT OF AN ENERGY \\ EFFICIENCY KNOWLEDGE CENTER (EEKC)}

by Seyed Mohammad Jalali

The Industrial Assessment Center (IAC) at West Virginia University has worked with more than 430 manufacturing companies over 17 years to identify energy and productivity saving opportunities for small and medium sized companies. The savings, which keep adding up year after year, are appreciable but do not fully capture the potential impacts of the IAC Program. The implementation rate of the recommendations has been only about $40 \%$ on average. This rate was expected to be improved with a knowledge center.

Energy Efficiency Knowledge Center (EEKC) is a regional system which includes the development and deployment of technical resources to assist industrial facilities in energy assessment phases. The EEKC as a repository for information and knowledge is designed to contribute to continuous improvement by incorporating the knowledge of IAC experts and plant personnel. The EEKC provides information to clients on how to obtain baseline energy use for their specific energy systems by fuel type using tools such as Quick Plant Energy Profiler (QuickPEP) by the Department of Energy (DOE).

The EEKC also incorporates access and utilization of the DOE Best Practices tools for enabling sensitivity analysis and estimating energy savings potential at various assessment phases. Moreover, spreadsheet tools are integrated within application software as required enabling clients to analyze the value of energy efficiency measures during the pre-assessment and assessment phases.

In this research thesis, the IAC activities and tasks are studied with respect to information and knowledge flow. The research objectives are presented and potential materials and steps to develop an online knowledge center are reviewed. Then, based on needs identified for continuous improvement, the design of EEKC is planned in which Web 2.0 is mainly used. 


\section{ACKNOWLEDGMENTS}

I would like to express my appreciation to my research advisor, Dr. Gopalakrishnan, for his support and guidance throughout my graduate studies at the Department of Industrial and Management Systems Engineering. I would also like to thank my committee members, Dr. Creese and Dr. Mohaghegh, for their support on my thesis.

I am sincerely grateful to my wife and my parents whose valuable supports and love motivate me not only to finish my master's degree but also to continue my education for a higher degree. I would also like to take this opportunity to thank my colleagues at the Industrial Assessment Center at West Virginia University. 


\section{TABLE OF CONTENTS}

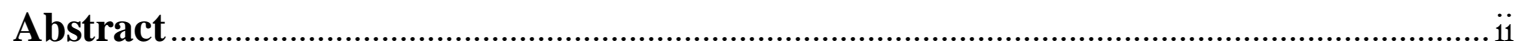

Acknowledgments..................................................................................................................... ii

TABLE OF CONTENTS ……………………………………………………………...... iv

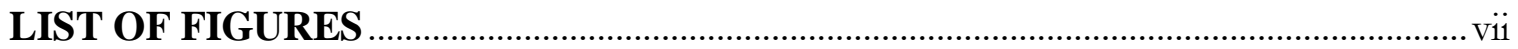

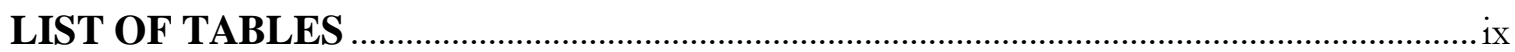

LIST OF SYMBOLS / NOMENCLATURE ……………………………………………….

CHAPTER 1: INTRODUCTION.....................................................................................

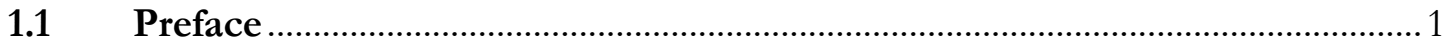

$1.2 \quad$ IAC Assessments …………………………………………………………2

$1.3 \quad$ IAC Main Tasks ...............................................................................................

1.4 SEN Assessment...........................................................................................

1.4.1 Pre-assessment Activities......................................................................... 6

1.4.2 Assessment Activities.....................................................................................

1.4.3 Post-assessment Follow-up ........................................................................

1.5 Continuous Improvement Plan............................................................................

1.6 Needs for a Knowledge Center to Improve the IAC Processes .........................10

$1.7 \quad$ Energy Efficiency Knowledge Center (EEKC) ……………………………….....11

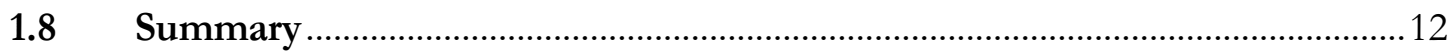

CHAPTER 2: LITERATURE REVIEW ....................................................................13

$2.1 \quad$ What is Knowledge?..........................................................................................13

$2.2 \quad$ Knowledge Management...................................................................................... 14

2.3 Knowledge Modeling Methods .......................................................................... 15

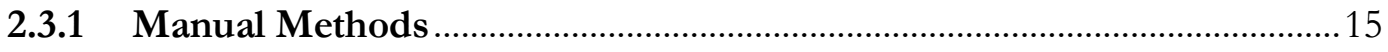

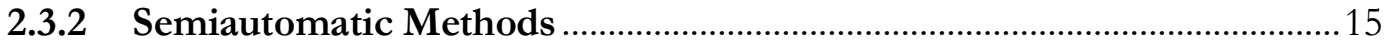

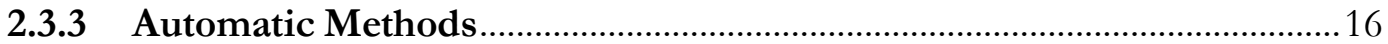

$2.4 \quad$ Knowledge-Engineering Process …………………………………………..... 16 


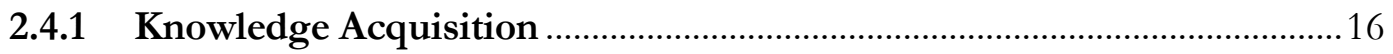

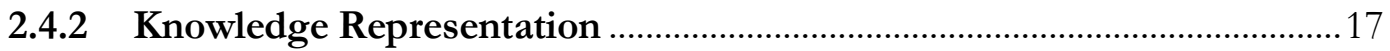

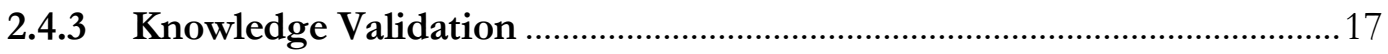

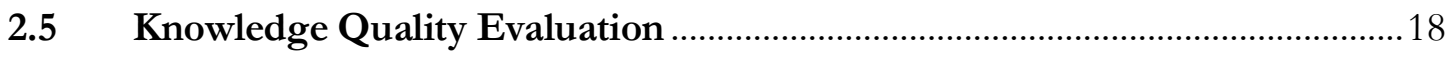

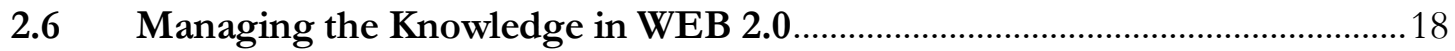

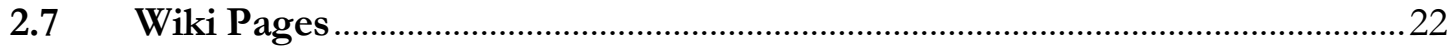

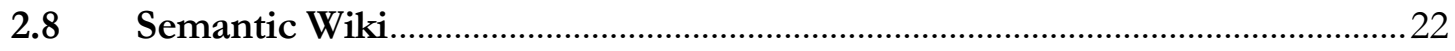

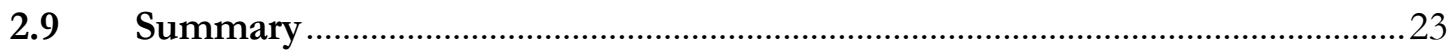

CHAPTER 3: THE IAC WORK PROCESSES, AND RELATED APPLICATIONS

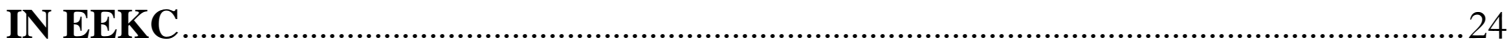

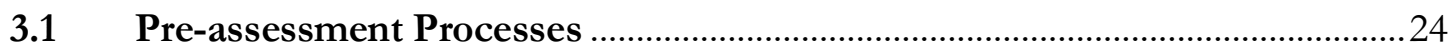

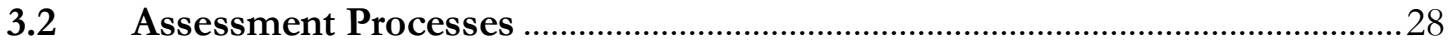

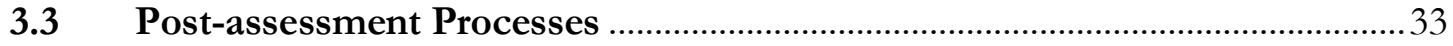

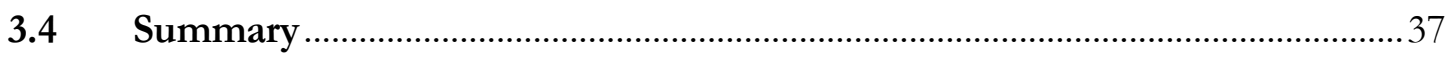

CHAPTER 4: DESIGN AND DEVELOPMENT OF THE EEKC.................................. 38

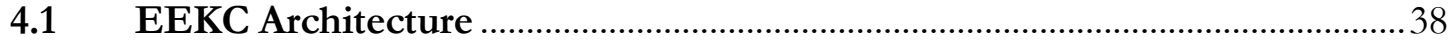

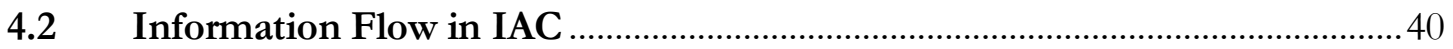

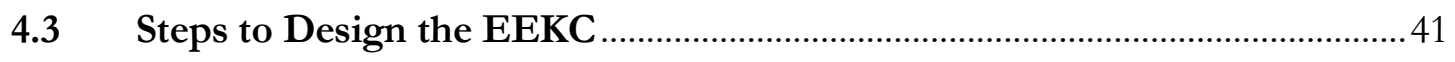

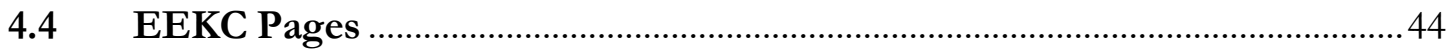

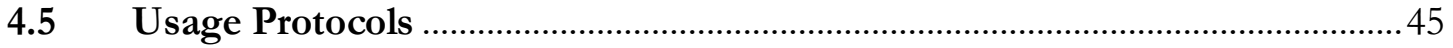

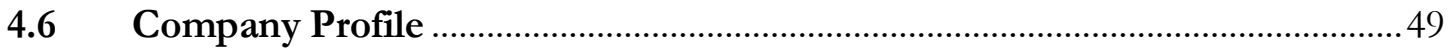

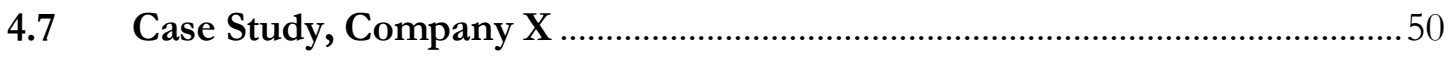

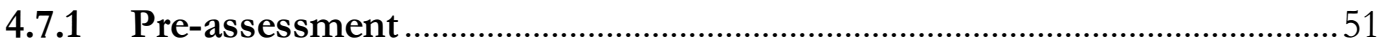

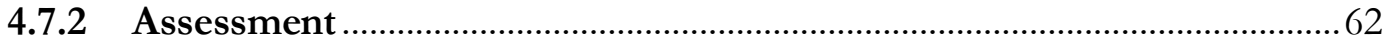

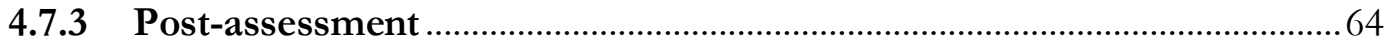

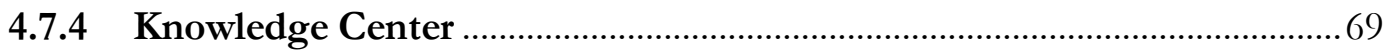

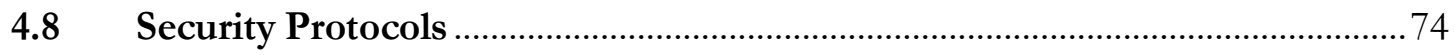

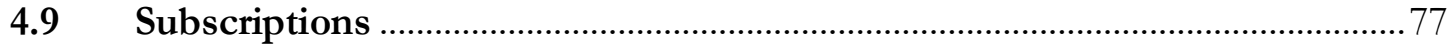

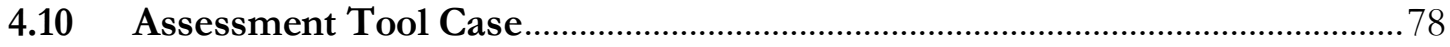

4.10.1 Air Compressor Checklists......................................................................... 


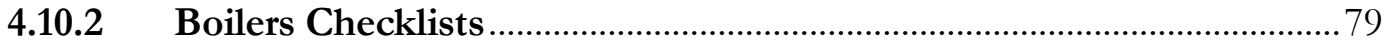

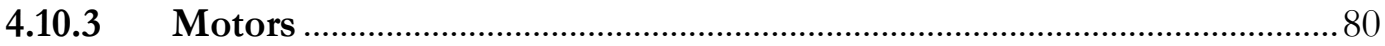

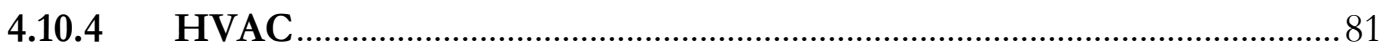

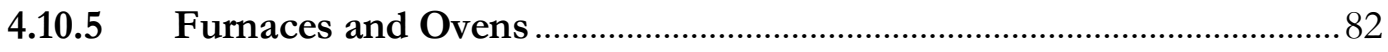

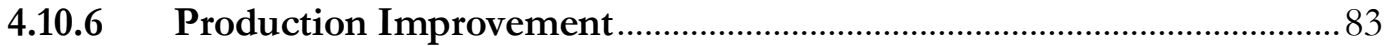

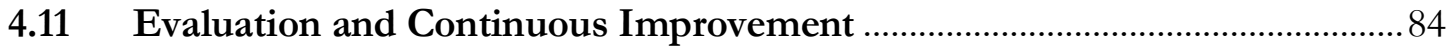

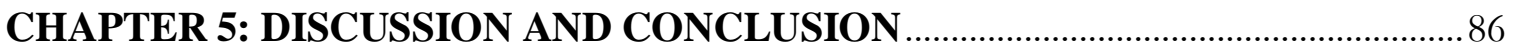

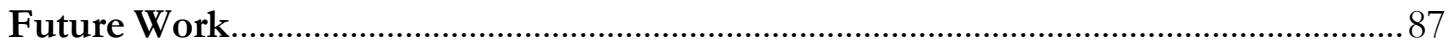

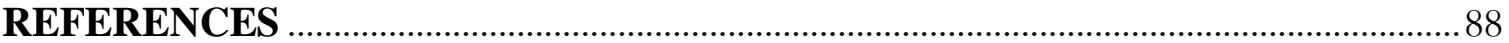




\section{LIST OF FIGURES}

Figure 1: Activities during the assessment phase ...............................................

Figure 2: Simplified process of knowledge engineering ............................................ 18

Figure 3: Three important factors to be considered in the EEKC architecture ..................39

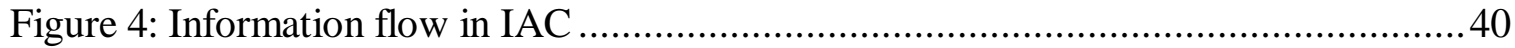

Figure 5: Steps to prepare the server and database .............................................. 41

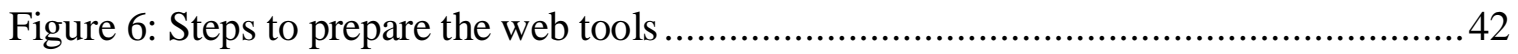

Figure 7: Enhancement and development steps .............................................. 43

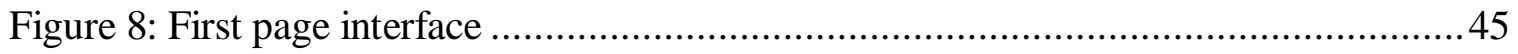

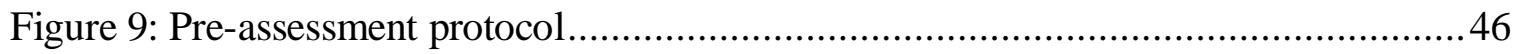

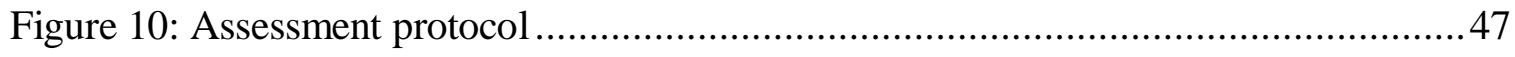

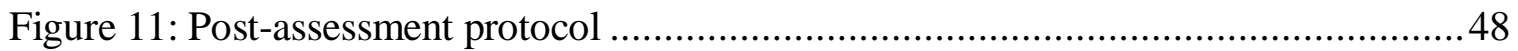

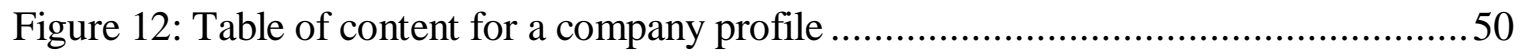

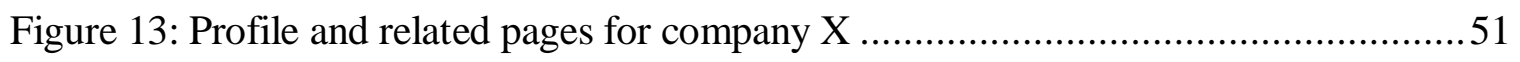

Figure 14: Company X's manufacturing processes .........................................54

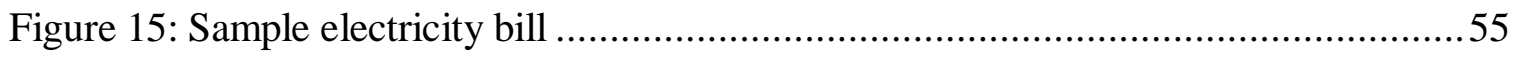

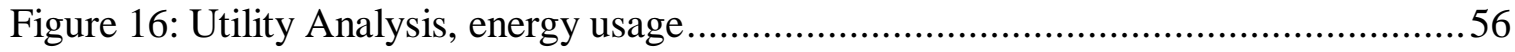

Figure 17: Utility Analysis, electricity cost.................................................5

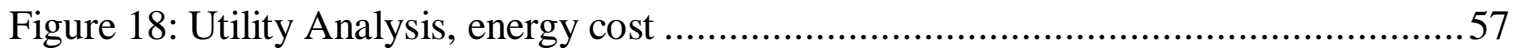

Figure 19: Energy consumption profile for the company X's plant.............................58

Figure 20: IAC Database, assessments for companies with steel product......................62

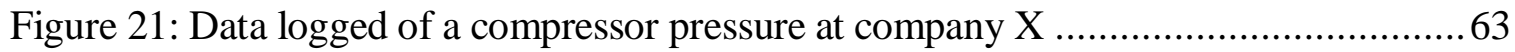


Figure 22: Current Profile of a compressor at company X............................................63

Figure 23: Pressure profile of a compressor at company X.........................................64

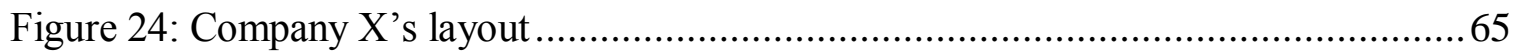

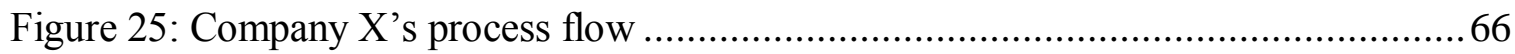

Figure 26: Demand and energy usage profile in 2009-2010 .........................................67

Figure 27: Average $\mathrm{kWh} / \mathrm{kW}$ distribution in 2009-2010 ...............................................6

Figure 28: Different categories presented in knowledge center page …...........................70

Figure 29: An example for AR bank (Insulation) ......................................................

Figure 30: A Wiki page, (Recover waste heat) ............................................................ 72

Figure 31: Showing a reference file directly in a Wiki page …....................................73

Figure 32: Attachment and comment illustration ........................................................74

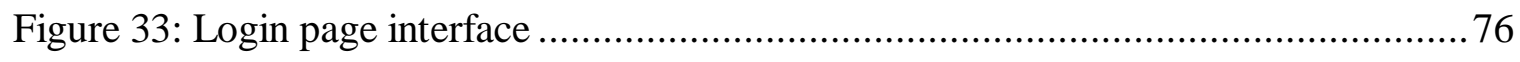

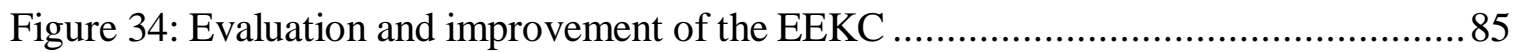




\section{LIST OF TABLES}

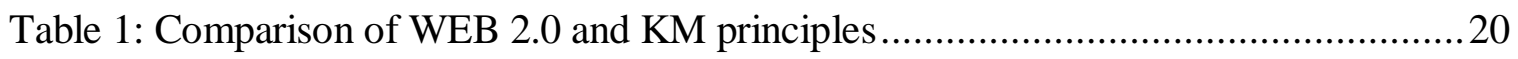

Table 2: Comparison of WEB 2.0 and KM tools and attributes ..................................2 21

Table 3: Information extracted from the questionnaire ........................................52

Table 4: Status of the previous ARs for company X, extracted from emails/discussion.....53

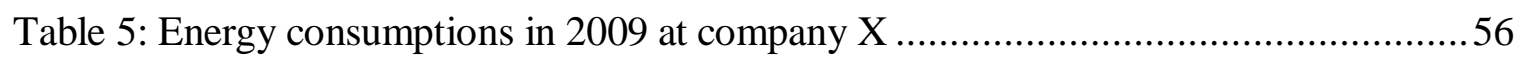

Table 6: Energy assessment results (summarized) …....................................... 65 


\section{LIST OF SYMBOLS / NOMENCLATURE}

\begin{tabular}{ll} 
AR & Assessment Recommendation \\
DOE & Department of Energy \\
ECM & Enterprise Content Management \\
EEAP & Enhanced Energy Assessment Process \\
EEKC & Energy Efficiency Knowledge Center \\
EEM & Energy Efficiency Measures \\
EERE & Energy Efficiency and Renewable Energy \\
FSAT & Fan System Assessment Tool \\
IAC & Industrial Assessment Center \\
ITP & Industrial Technologies Program \\
PHAST & Process Heating and Survey Assessment Tool \\
PSAT & Pump System Assessment Tool \\
QuickPEP & Quick Plant Energy Profiler \\
SEN & Save Energy Now \\
SSAT & Steam System Assessment Tool \\
SSL & Secure Sockets Layer \\
SSST & Steam System Scoping Tool \\
WCM & Web Content Management \\
WVU & West Virginia University \\
\hline
\end{tabular}




\section{CHA P TER 1: IN TRODUCTION}

\subsection{Preface}

Industrial Assessment Centers (IAC) are a part of industry wide effort towards increasing resource productivity through a process called Industries of the Future. This process, supported by the U.S Department of Energy's Industrial Technologies Program (ITP), focuses on energy and resource intensive materials and processing sector, accelerates research and development of advanced technologies identified as priorities by industry [1]. IACs provide eligible small and medium-sized manufacturers with no-cost energy assessments [2]. The outcomes of the IAC program are energy savings, resource efficiency and a trained workforce for addressing energy challenges, waste reduction, and productivity improvement.

Continuous improvement in work processes and assessment reports is one of the main missions of the IAC program [2]. It is expected that managing information and knowledge within the IAC program enhances the continuous improvement process. To manage the information and knowledge, a dynamic and secured system is needed in which information and knowledge could be saved, maintained and shared.

This research project aims to use appropriate tools to design and develop the knowledge center for the energy efficiency projects at the IAC. Research outputs and objectives of the research are as follows:

\section{- Research Outputs}

- An online system designed as the document and knowledge center

- Protocols to use the EEKC in energy efficiency assessments 


\section{- Research Objectives}

- Extract the knowledge from the regular energy assessment reports and prepare it to be entered into the knowledge center.

- Save and share the obtained knowledge.

- Archive, maintain, and manage the documents.

-Engage the IAC members to interact with the knowledge center.

-Improve the IAC work processes using the manual protocols; the manual protocols would be a recipe for the IAC members to use in assessment projects.

- Manage the information and knowledge flow in work processes using the protocols and the knowledge center.

This research thesis reviews the IAC activities, tasks, and work processes, and then reviews the literature and tools used to design the knowledge center. It then continues with research methodology and ends up with future work.

\subsection{IAC Assessments}

IAC assessments are performed by local teams of engineering faculty and students from 26 participating universities across the country. The assessment begins with a university-based IAC team conducting a survey of the eligible plant, followed by a one or two-day site visit, taking engineering measurements as a basis for assessment recommendations. The team then performs a detailed analysis for specific recommendations with related estimates of costs, performance and payback times [2]. 
The IACs use a variety of tools and techniques that enable them to be successful in performing the energy assessments as well as maintain high level of energy saving metrics. The DOE Best Practices software tools [2] such as Process Heating and Survey Assessment Tool (PHAST), AirMaster+, Fan System Assessment Tool (FSAT), Pump System Assessment Tool (PSAT), Steam System Assessment Tool (SSAT) and Steam System Scoping Tool (SSST) are being utilized by the IACs in evaluating the potential of energy assessment recommendations. In addition, many of the IACs use custom made spreadsheet tools and other software tools that help in this regard.

The IACs perform energy assessment for various manufacturing facilities and may focus on crosscut areas with respect to energy systems or devote effort entirely on specific energy systems. The decision in this regard is usually made based upon energy intensity associated with specific energy systems in the facility. For example, if steam system in a facility consumes over $80 \%$ of the energy, then it becomes a good candidate for system based energy assessment.

During the assessments, the IAC teams also introduce the plant personnel to some of the equipment and Best Practices software used in the assessment, and provides them with limited training. Figure 1 illustrates the assessment main activities.

Within 60 days, a confidential report, detailing the analysis, findings and recommendations of the team is sent to the plant [2]. In two to six months, follow-up phone calls are placed to the plant manager to verify recommendations that are implemented. 


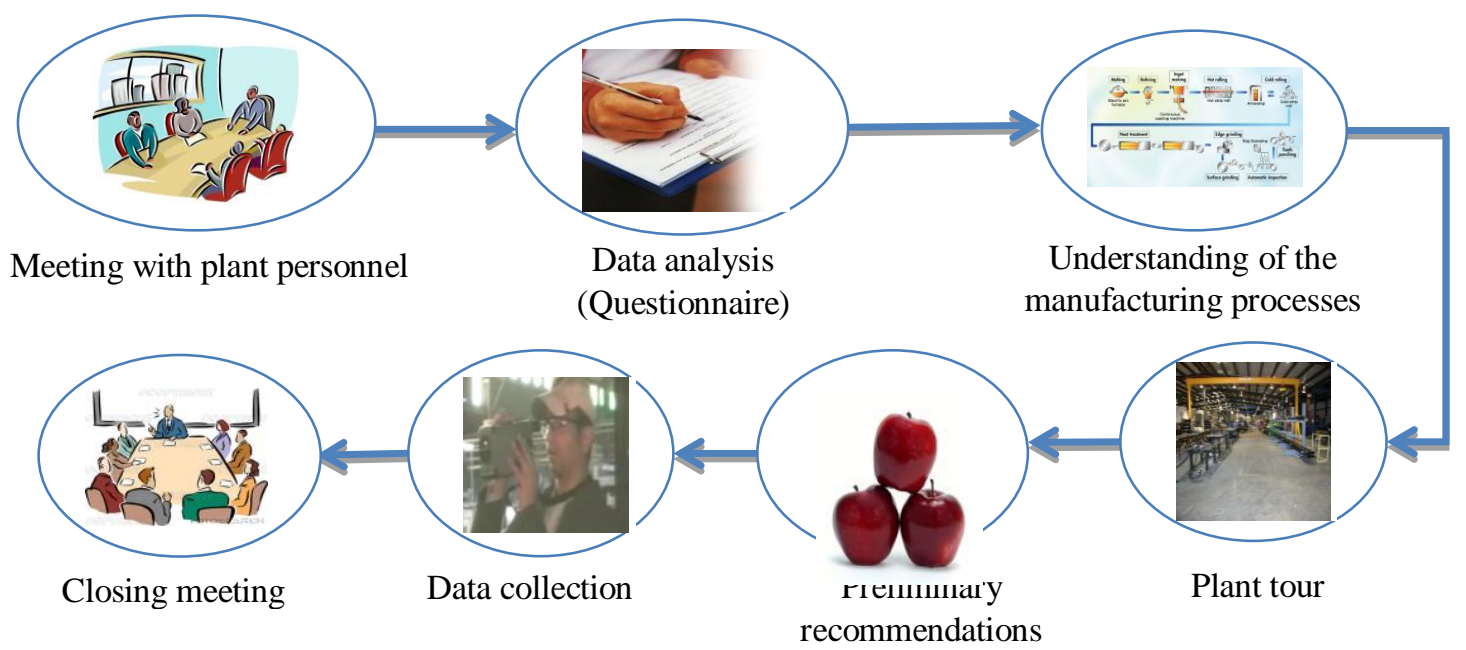

Figure 1: Activities during the assessment phase

\subsection{IAC Main Tasks}

For designing the Energy Efficiency Knowledge Center (EEKC), it is critical to study IAC's work flow and tasks. Following is a short list of the IAC main tasks:

- Scheduling the assessment: Identify potential clients, contact the client, and establish assessment date.

- Pre-assessment tasks: Create client file, check energy bills for completion, obtain directions, record assessment information on IAC spreadsheet, confirm assessment visit, and check equipment and data collection documents.

- Site visit: Opening meeting, collect general information, plant tour, develop recommendations, collect field data, and closing meeting.

- Report Process: Assessment Recommendation (AR) assignments, complete energy bill analysis section, complete facility background section, complete AR assignments, integrate Best Practices software use into ARs, merge document, initial document review, make corrections, final document review, final document merge, mail 
electronic and hard copies of report, and upload documents to Field Manager's database (IAC database).

- Contact client on receipt of report and answer any preliminary questions.

- Respond to critiques from Field Manager on report.

- Weekly coordination meetings

- Quarterly reporting

- Implementation survey and report

- Post-implementation follow up calls to clients and determine additional energy savings metrics

In addition to the regular IAC assessment, Save Energy Now (SEN) is another form of assessments at the IAC which is explained in the following section.

\subsection{SEN Assessment}

Unlike the standard IAC assessment, which concentrates on the on-site visit and the production of the assessment report, the SEN assessment process pays attention to, and provides services at, varying stages of the assessment process, namely, pre-assessment, assessment, and post-assessment with a strong focus on enhancing the implementation of Energy Efficiency Measures (EEM). The pre- and post-assessment processes, developed by the IACs and their energy efficiency and best practices knowledge, play a major role in delivering and ensuring a continuous energy conservation awareness and policy amongst manufacturers. SEN assessment activities include the following activities: 


\subsubsection{Pre-assessment Activities}

The pre-assessment phase includes interactive sessions via e-mail or a phone with plant personnel to familiarize them with the assessment process, Best Practices software tools, overview of energy systems, and baseline energy use analysis. The pre-assessment data collection and data analysis is done to understand the baseline energy use and to identify those areas that may benefit the most from potential retrofits. It also helps the assessment team develop the plan of audit for each individual facility based on the process characteristics and energy use.

After pre-assessment preparations to identify a potential client, a telephone contact is made and an appropriate person identified as the company's representative, typically the plant manager, maintenance manager, or engineering manager. Prior to the plant site visit, the company's representative is asked to provide the IAC with a copy of the 12 most recent months of energy bills, including electricity, natural gas, fuel oil, and any other fuel that is used to provide energy for the facility. The company is also asked to provide the IAC with relevant data on waste disposal quantities that may have an energy impact, and their associated costs. The company is encouraged to send a brochure and other descriptive information about its products and operations. A questionnaire is then sent to the company requesting information in detail about the operational characteristics and data on product, process, and systems parameters as well as utility cost and usage.

\subsubsection{Assessment Activities}

The assessment process focuses on energy analysis and diagnostics, interdependence between energy efficiency recommendations, and sensitivity analysis 
with respect to key products, processes, and system parameters governing energy savings. Based on the results of the pre-assessment phase, specific energy consuming systems are usually targeted. The set of diagnostic tools is chosen based on the systems to be investigated and parameters to be measured or monitored.

At the plant, the assessment will start with an opening meeting with appropriate plant personnel, during which the assessment process, DOE/ITP services, DOE sponsorship, confidentiality of information, implementation aspects and the reporting process will be explained to the client. The information collection process then moves into high gear as data are gathered about the operations and manufacturing processes of the company, the types of equipment used in the facility such as air compressors, heating/air conditioning units, boilers, chillers, other significant energy consuming equipment, and the information on the types of waste generated by the plant and productivity information that may have an energy impact. The opening meeting is followed by a plant tour, which is conducted in the order of the production process from raw material storage to shipment of finished goods. During this plant tour each IAC team member will observe and record his/her ideas for potential recommendations.

After the tour, the assessment group will meet and discuss the opportunities that were found, and have a brainstorming session to develop additional ideas. Members of the group will determine the best opportunities for savings, and then organize to collect the required information using the appropriate instrumentation and data sheets. The IAC team will collect pertinent information by returning to the plant floor to take measurements where needed, ask questions of plant personnel when appropriate, observe 
the operation, and collect data. The entire group will then meet again to discuss and document the potential assessment recommendations.

The extended visits are realized in the form of additional time spent at the plant (two-day assessment), return visit (if necessary), and/or a presentation made to the plant personnel. The duration of energy audit and data collection periods are selected to ensure availability and reliability of data for sound analysis and generation of energy saving recommendations. Additional visits are usually scheduled if more information about specific systems is required or when there is a need to discuss sources of funding or rebates available for a given energy conservation measure.

In the scope of SEN Assessment, WVU-IAC makes a commitment to develop process-specific energy conservation recommendations that may apply only for a particular facility. To this end, the database of ITP and Best Practices Tools are utilized extensively. New technologies available for a given manufacturing sector are explored to give the customers important information about the availability of alternative or more efficient processes and technologies.

\subsubsection{Post-assessment Follow-up}

The post-assessment activities may focus on enhancing the relationship with client companies and providing technical information, measurement for verification of energy savings, life cycle cost analysis of recommendations, implementation assistance, training, input on monitoring energy systems, financial assistance information for implementation, and discussion for removal of barriers for implementation. Investigation 
of available rebates and incentives from utility companies or federal and state agencies for each energy conservation measure are also provided.

To help the plant with implementation of the energy conservation measures, a survey of the equipment available in the market is conducted. This survey results in the short report outlining the technical specifications and operating parameters of the applicable equipment as well as cost data. No vendor or brand names are mentioned in the report.

Different states have different portfolio of rebates and incentives (tax credits, deductions, depreciation schedules) available for industrial facilities. In the scope of SEN Assessments, WVU-IAC conducts a thorough analysis of these portfolios to identify any financial benefits that a given plant may get in respect to a particular energy conservation measure.

The post-assessment site visits may be scheduled based on the implementation status obtained from the plant personnel.

\subsection{Continuous Improvement Plan}

The IACs strive to continuously improve their operations. The IAC students and Directors attend training sessions and attempt to become qualified specialists in specific areas. These activities strengthen the efforts to integrate the DOE's Office of EERE and ITP Best Practices with the IAC activities and reports submitted to industrial clients. These activities also lead to the development of new assessment recommendations, help 
in increasing the savings expected from the proposed recommendations as well as the implementation ratios, and enhance the quality of the reports.

\subsection{Needs for a Knowledge Center to Improve the IAC Processes}

Continuous improvement in energy assessments requires that learning from past and current projects be transferred on to future energy assessments. The learning in the assessments, which is generally called as knowledge, needs to be captured, validated and shared among the IAC members to use in the assessments and the IAC work processes. Moreover, it is essential that all the IAC members use a particular online system to save, add, and manage versions of the ARs, spreadsheets, etc. Currently, most of the files are archived offline on the IAC computers.

Each AR usually has different templates and each template is enhanced in different assessments and gets a new version; therefore, it is vital to save and maintain the ARs on a unique online system which is connected to discussion forums, and knowledge and hands on experiences available in the knowledge center.

A knowledge based systems would be an appropriate tool to save, evaluate, and share not only learning from the assessments but also data and information gathered in each particular assessment project. The following section shortly presents the knowledge center designed for the energy efficiency assessments. The knowledge center is more explained in the following chapters. 


\subsection{Energy Efficiency Knowledge Center (EEKC)}

The EEKC will be a repository for information and knowledge generated by the regional Enhanced Energy Assessment Process (EEAP) at IACs. The EEAP has been developed as a result of the SEN assessments. The targeted region to use the EEKC includes West Virginia, western Pennsylvania and eastern Ohio. The expanded region also includes Tennessee and Kentucky.

As it is mentioned in Section 1.4, the primary objective of the SEN assessments is to identify and evaluate opportunities for energy conservation as well as to help companies with implementation and verification phases.

The EEKC will utilize database driven Internet protocols containing energy efficiency knowledge specific to each of the major energy systems. Clients from industrial facilities will be able to access the EEKC via the Internet under secured conditions to obtain knowledge about the results obtained in the assessment process as well as other best practices knowledge gained by the IAC and SEN experience of the project participants. The EEKC will provide information and knowledge to clients on how to obtain baseline energy use for their specific energy systems by fuel type using tools such as Quick Plant Energy Profiler (QuickPEP).

Typical energy efficiency recommendations for energy systems will be available for review, as well as information on accessing the IAC database for understanding the extent of recommended and implemented energy savings. Spreadsheet tools will be integrated within application software as required enabling clients to analyze the value of EEM during the pre-assessment and assessment phases. A database approach will be used to provide knowledge on the details regarding implementation aspects in the post-assessment phase 
for EEM, including cost, time, and process constraints. The tools used to design the EEKC are reviewed in Chapter 2.

\subsection{Summary}

The IAC assessments help small- and medium-sized manufactures save energy and reduce waste. Currently, the implementation rates of the ARs are about $40 \%$ on average. It was expected that the EEKC could increase the implementation rates by improving the IAC internal work processes and managing information and knowledge flow. The EEKC is going to provide solutions to extract, storage, and share the energy efficiency knowledge. Besides being a knowledge center and a virtual assistant for the IAC members, some protocols would help the IAC members use the EEKC and manage information and knowledge within their activities.

The rest of this thesis is organized as follows: The literature review is presented in Chapter 2. Chapter 3 reviews the IAC work processes and related applications. Then Chapter 4 presents the necessary tools and information to develop the EEKC. Finally the thesis ends up with discussion and conclusion, and future work suggestions. 


\section{CHA P TER 2: LITERATURE REVIEW}

The IAC characteristics and SEN assessments were reviewed in introduction chapter. In this chapter the basic materials for design and development of the EEKC are studied. This chapter is organized as follows: First, the general concepts of knowledge and knowledge management are reviewed. Then, knowledge modeling methods and knowledge engineering process are studied. The literature review chapter continues with study of Web 2.0 tools and Wiki pages, and their applications in EEKC.

\subsection{What is Knowledge?}

There are various definitions of knowledge. In general, knowledge can be defined as follows [3]:

Knowledge is the $\left\{\begin{array}{c}\text { ability } \\ \text { skill } \\ \text { expertise }\end{array}\right\}$ to $\left\{\begin{array}{c}\text { manipulate } \\ \text { transfer } \\ \text { create }\end{array}\right\}\left\{\begin{array}{c}\text { data } \\ \text { Information } \\ \text { ideas }\end{array}\right\}$ to $\left\{\begin{array}{c}\text { perform skillfully } \\ \text { make decisions } \\ \text { solve problems }\end{array}\right\}$

Knowledge can only be completely understood if two things are known [3]: (1) How it is structured, i.e. what components it is made from, and the ways they are linked together; (2) How it operates, i.e. the ways in which the components behave and the processes that are happening. In general, knowledge can be categorized as either explicit knowledge or tacit knowledge [4].

\subsubsection{Explicit Knowledge}

Explicit knowledge is the type of knowledge that an individual has acquired mainly in school and university. Explicit knowledge implies factual statements about such matters as 
material properties, technical information, and tool characteristics. Thus, explicit knowledge can be expressed in words and numbers, and is therefore easily communicated and shared [5].

\subsubsection{Tacit Knowledge}

Tacit knowledge is highly personal and hard to communicate or to share with others. Tacit knowledge is deeply rooted in an individual's experience, and it consists of schemata, belief, and perceptions stored so deep in the worldview of an individual that we take them for granted [5].

\subsection{Knowledge Management}

Knowledge Management (KM) is a discipline focused on systematic and innovative methods, practices, and tools for managing the generation, acquisition, exchange, protection, distribution, and utilization of knowledge, intellectual capital and intangible assets [6]. KM is a system of actions upon knowledge, which includes the establishment of strategies and procedures, with proper utilization of technologies, so that the acquisition, storage, conversion, sharing, application, and generation of knowledge can be effectively performed, with an aim to effectively use the available knowledge for problem solving and decision making [7].

Often knowledge sharing is the core intent of a $\mathrm{KM}$ initiative, so it has become an important theoretical and practical subject [8]. Some examples of KM applications for knowledge sharing are listed below [9]:

- Document and Content Management systems 
- Knowledge Maps

- Intelligent Agents

- Web Browsers

- Business Intelligence Tools and Data Warehouses

- Workflow Applications

- E-learning and Collaboration Tools

\subsection{Knowledge Modeling Methods}

Knowledge modeling methods can be classified into three categories, manual, semiautomatic and automatic described as follows [10]:

\subsubsection{Manual Methods}

Manual methods are basically structured around an interview of some kind. The knowledge engineer elicits knowledge from the expert or other sources and then codes it in the knowledge base. The three major manual methods are interviewing (i.e., structured, semi-structured, unstructured), tracking the reasoning process, and observing. Manual methods are slow, expensive, and sometimes inaccurate. Therefore, there is a trend toward automating the process as much as possible.

\subsubsection{Semiautomatic Methods}

Semiautomatic methods are divided into two categories: those intended to support the experts by allowing them to build knowledge bases with little or no help from knowledge engineers and those intended to help knowledge engineers by allowing them 
to execute the necessary tasks in a more efficient or effective manner (sometimes with only minimal participation by an expert).

\subsubsection{Automatic Methods}

In automatic methods, the roles of both the expert and the knowledge engineer are minimized or even eliminated. For example, the induction method, which generates rules from a set of known cases, can be applied to build a knowledge base. The roles of the expert and knowledge engineers are minimal. The term automatic may be misleading, but

it indicates that, compared with other methods, the contributions from a knowledge engineer and an expert are relatively small. The automatic method is used to develop the EEKC.

\subsection{Knowledge-Engineering Process}

Knowledge Engineering involves integrating knowledge into computer systems in order to solve problems. Knowledge engineering process initiates with the following major activities [10]:

\subsubsection{Knowledge Acquisition}

Knowledge acquisition involves the acquisition of knowledge from human experts, books, documents, sensors, or computer files. The knowledge may be specific to the problem domain or to the problem-solving procedures, it may be general knowledge e.g. knowledge about business, or it may be metaknowledge (knowledge about knowledge). 
Knowledge acquisition is the transformation of potential problem solving expertise from some knowledge sources to a program. Knowledge acquisition includes the elicitation, collection, analysis, modeling and validation of knowledge for knowledge engineering and KM projects [3].

To create a knowledge base requires knowledge to be captured. There are a number of important factors that should be considered in knowledge acquisition such as [3]:

- The end-product must be useful to the end-users;

- To be useful, the end-product must be full of high-quality knowledge that is correct, complete and relevant and that is stored in a structured manner;

- The project must be run in an efficient way making the most use of the available resources;

- The project should not unduly disrupt the normal running of the organization, hence should not involve too much time from experts.

\subsubsection{Knowledge Representation}

Acquired knowledge is organized so that it will be ready for use, in an activity called knowledge representation. This activity involves preparation of a knowledge map and encoding of the knowledge in the knowledge base.

\subsubsection{Knowledge Validation}

Knowledge validation (or verification) involves validating and verifying the knowledge, e.g. by using test cases, until its quality is acceptable. The concept of knowledge validation is more explained in the following Section. 


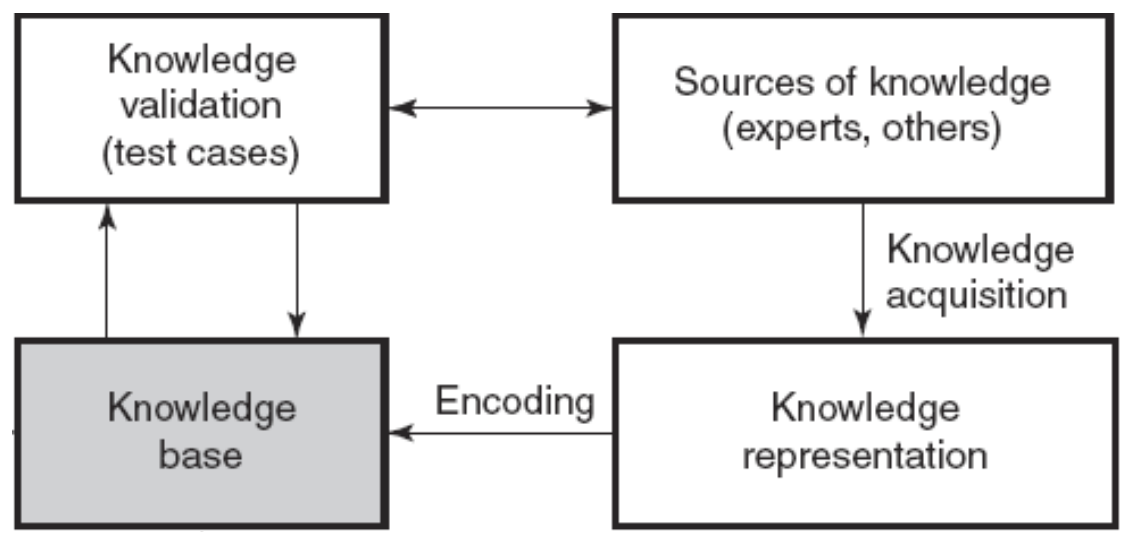

Figure 2: Simplified process of knowledge engineering [10]

\subsection{Knowledge Quality Evaluation}

Knowledge acquired from experts and other sources needs to be evaluated for quality, including evaluation, validation, and verification. These terms are often used interchangeably. They are explained as follows [10]:

- Evaluation is a broad concept. Its objective is to assess knowledge's overall value. In addition to assessing acceptable performance levels, it analyzes whether the knowledge would be usable, efficient, and cost-effective.

- Validation is the part of evaluation that deals with the performance of the knowledge. Simply stated, validation is building the right knowledge.

- Verification is building the knowledge structure right or substantiating that the knowledge is correctly settled to its specifications.

\subsection{Managing the Knowledge in WEB 2.0}

The term "Web 2.0" refers to the second generation development and design of the web that aims to facilitate communication and to secure information sharing, interoperability 
and user centered design. Web 2.0 tools facilitate sharing, networking and disseminating information among friends and other professional groups. Web 2.0 applications encourage patrons to be an integral part of the virtual community by sharing their ideas, thoughts, and other content [11].

The principles of WEB 2.0 and KM are very similar (analyzed in Table 1). In order to compare WEB 2.0 to $\mathrm{KM}$ on the functional level, Table 2 is a table analyzing components of the WEB 2.0 tools, attributes, and their reference in the classical KM tools. The comparison is based on functional aspects and does not refer to infrastructure aspects [12]. Web 2.0 and KM tools and attributes are compared in Table 2.

Summing up the attributes comparison analyzed in Table 2, most WEB 2.0 tools attributes have roots in KM tools. Many of the features already exist, even though there are differences between the two [12]. Among the Web 2.0 components studied in Table 2, Wiki is chosen to use as the main part in the EEKC. Wiki is then more studied in the following Section. 
Table 1: Comparison of WEB 2.0 and KM principles [12]

\begin{tabular}{|c|c|}
\hline $\begin{array}{l}\text { WEB 2.0 } \\
\text { principle }\end{array}$ & KM matching principle \\
\hline $\begin{array}{l}\text { 1. WEB as a } \\
\text { platform }\end{array}$ & $\begin{array}{l}\text { Technology as a platform } \\
\text { The KM world is based on four complementary components: culture, process, } \\
\text { technology and content which none of them is independent. In their book, Working } \\
\text { Knowledge, Davenport and Prusak emphasize this principle: "effective KM cannot take } \\
\text { place without extensive behavioral, cultural and organizational change". }\end{array}$ \\
\hline $\begin{array}{l}\text { 2. Services } \\
\text { development }\end{array}$ & $\begin{array}{l}\text { WEB services } \\
\text { WEB services, is the most popular way for sharing data and information, context related, } \\
\text { in portal window-lets and in the portal professional desktop. } \\
\text { In the KM world, ones does not care (for ideological reasons) where the information is } \\
\text { stored, rather how it is used in various knowledge applications. The portal is a broker, } \\
\text { via which services present the data, information and knowledge. }\end{array}$ \\
\hline $\begin{array}{l}\text { 3. Active } \\
\text { participation of } \\
\text { users }\end{array}$ & $\begin{array}{l}\text { Active participation of users } \\
\text { KM deals with sharing the knowledge and preserving it. The knowledge is based on } \\
\text { users, and without them, such activities cannot take place. Active participation of users is } \\
\text { a necessity. } \\
\text { Nevertheless, in KM, the users' participation is encouraged by a central team usually } \\
\text { convincing people to add content; in many cases, they will even settle with users only } \\
\text { using knowledge (built by several key users). Sharing is controlled. In some cases, } \\
\text { content added is moderated before published. } \\
\text { In WEB 2.0, by comparison, activities are decentralized and people add voluntarily. }\end{array}$ \\
\hline $\begin{array}{l}\text { 4. The service } \\
\text { improves } \\
\text { automatically, } \\
\text { the more it is } \\
\text { used. }\end{array}$ & $\begin{array}{l}\text { Partly correct in KM } \\
\text { Of course, if people participate more, there is more content, and richer content, adding } \\
\text { value to service offered to the user. But, this cannot be compared to the situation of } \\
\text { WEB } 2.0 \text { applications. In WEB } 2.0 \text { the software itself is based on automatic } \\
\text { improvement the more it is used. }\end{array}$ \\
\hline $\begin{array}{l}\text { 5. Collective } \\
\text { intelligence }\end{array}$ & $\begin{array}{l}\text { KM is based on the collective knowledge of its users. Nonaka and Takeuchi in their } \\
\text { book, The Knowledge Creating Company, described the success of the Japanese } \\
\text { companies in developing knowledge, based on the Japanese sharing culture which builds } \\
\text { the collective organizational new knowledge. } \\
\text { A major difference between the two has to do with dealing with the LONG TAIL } \\
\text { concept. WEB } 2.0 \text { sanctifies it, KM ignores it. KM solutions are based, in many cases, } \\
\text { on a mass of } 20 \text { percent of the users (content experts), contributing } 80 \text { percent of the } \\
\text { knowledge. }\end{array}$ \\
\hline $\begin{array}{l}\text { 6. Content as } \\
\text { the core }\end{array}$ & $\begin{array}{l}\text { Content is one of the four components on which KM stands: culture, processes, } \\
\text { technology and content. It does not stand for itself, yet it is part of the core, and no KM } \\
\text { solution can take place without a rich content segment. } \\
\text { A decade ago, when KM was initiated, it was not yet understood that content drives KM. }\end{array}$ \\
\hline $\begin{array}{l}\text { 7. The perpetual } \\
\text { beta }\end{array}$ & $\begin{array}{l}\text { At first glance the concept of perpetual beta may seem irrelevant to KM. } \\
\text { KM does not deal with technology, but KM uses technology. Working with a perpetual } \\
\text { beta, can serve KM a great deal. One of the KM challenges has to do with understanding } \\
\text { this potential. Organizations can design communities of practice, portals and knowledge } \\
\text { sites with care and thought, yet only after launch and use, do people realize what more } \\
\text { can be added in. Potential is learnt via use. Changes are required frequently, adjusting } \\
\text { the technology to the people using it as they and their needs mature. The perpetual beta } \\
\text { is certainly a great enabler. }\end{array}$ \\
\hline
\end{tabular}


Table 2: Comparison of WEB 2.0 and KM tools and attributes [12]

\begin{tabular}{|c|c|c|c|}
\hline $\begin{array}{c}\text { WEB } 2.0 \\
\text { component }\end{array}$ & Attribute & $\begin{array}{l}\text { Relevant attribute in } \\
\text { knowledge management }\end{array}$ & Gaps \\
\hline WIKI & $\begin{array}{l}\text { Structured } \\
\text { content pages }\end{array}$ & $\begin{array}{l}\text { Web Content Management } \\
\text { (WCM) tools. These tools are } \\
\text { part of the KM toolbox and; } \\
\text { Are used for rich content sites. }\end{array}$ & $\begin{array}{l}\text { WIKI: is known for its user friendly } \\
\text { usage; Is flexible, both content and } \\
\text { structure wise; Includes a high level of } \\
\text { connectivity between items (also in } \\
\text { free text); Deals with homogeny sites. }\end{array}$ \\
\hline $\mathrm{B} \log$ & $\begin{array}{l}\text { Personal diary, } \\
\text { including access } \\
\text { to the diary as a } \\
\text { whole. } \\
\text { Enables fast } \\
\text { access to new } \\
\text { pages, with } \\
\text { easy access to } \\
\text { history }\end{array}$ & $\begin{array}{l}\text { Can be implemented using a } \\
\text { variety of KM tools, whether } \\
\text { Enterprise Content Management } \\
\text { (ECM) tools or portal style tools; } \\
\text { The solution provided by Blogs } \\
\text { reminds one of another KM tool } \\
\text { (physical, not virtual): } \\
\text { Storytelling. }\end{array}$ & $\begin{array}{l}\text { The innovation of WEB } 2.0 \text { depicted } \\
\text { in Blogs, is not mainly the idea, but in } \\
\text { the way it is implemented: Very } \\
\text { simple, very accessible, and therefore, } \\
\text { very appealing. Therefore, it is easy to } \\
\text { understand that the idea itself can be } \\
\text { copied, using various KM tools. }\end{array}$ \\
\hline RSS & $\begin{array}{l}\text { Alerts regarding } \\
\text { new content } \\
\text { items and } \\
\text { changes among } \\
\text { existing ones, by } \\
\text { categories }\end{array}$ & $\begin{array}{l}\text { Alerts are a known and well used } \\
\text { mechanism in ECM tools as well } \\
\text { as in Portals. Search engines } \\
\text { enable running fixed searched, } \\
\text { giving the same results as alerts; } \\
\text { Another tool informing users } \\
\text { about alerts is a common web-let } \\
\text { of "what's new" in almost every } \\
\text { organizational and professional } \\
\text { portal. }\end{array}$ & $\begin{array}{l}\text { The gap is mainly in two aspects: (1) } \\
\text { The way the information is packaged. } \\
\text { Instead of receiving alerts separately } \\
\text { from every information resource, } \\
\text { information is packaged together; } \\
\text { (2) The user does not have to point to } \\
\text { all sources. Requesting information } \\
\text { and knowledge is defined via its } \\
\text { categories. }\end{array}$ \\
\hline Tagging & $\begin{array}{l}\text { Everyone can } \\
\text { subjectively tag } \\
\text { his or her own } \\
\text { information }\end{array}$ & $\begin{array}{l}\text { Tags are provided in several } \\
\text { tools: In portals - via navigation } \\
\text { menus; } \\
\text { In search engines - via filtering } \\
\text { attributes; } \\
\text { In ECM tools - by both of the } \\
\text { above; } \\
\text { Yet, all these are built as part of } \\
\text { taxonomy, either organizational } \\
\text { or departmental, trying to be } \\
\text { objective (inside the group) as far } \\
\text { as possible. }\end{array}$ & $\begin{array}{l}\text { In WEB } 2.0 \text { each page can be tagged } \\
\text { more than once, and not only by the } \\
\text { author; } \\
\text { In WEB } 2.0 \text { the tagging is subjective } \\
\text { as opposed to KM, where it is } \\
\text { objective; } \\
\text { In WEB } 2.0 \text { there are no pre-defined } \\
\text { lists of attributes and values defining } \\
\text { the "'allowed" tags. }\end{array}$ \\
\hline $\begin{array}{l}\text { Social } \\
\text { computing }\end{array}$ & $\begin{array}{l}\text { Building social } \\
\text { communities } \\
\text { over the net }\end{array}$ & Communities of practice & $\begin{array}{l}\text { Most communities on WEB } 2.0 \text { are } \\
\text { based on personal hobbies and fields } \\
\text { of interest. Examples include } \\
\text { del.ico.us (bookmarks), Youtube } \\
\text { (video films) and Flickr (photos); } \\
\text { Communities of practice, comparing, } \\
\text { are focused on professional issues. In } \\
\text { many cases they are complemented by } \\
\text { face-to-face gathering, which is rare } \\
\text { on WEB 2.0; } \\
\text { Both environments give } \\
\text { supplementing tools such as sharing } \\
\text { files and discussions, all by categories. }\end{array}$ \\
\hline
\end{tabular}




\subsection{Wiki Pages}

A Wiki is a website, which facilitates the creation and editing of web pages using a simplified markup language based text editor, and is used to create collaborative websites. Anyone with a web browser can edit content of Wikis (with respect to security levels), which are organized by Wiki software [11].

Wiki is often described as one of the most popular Web 2.0 technologies. A Wiki enables communities to write documents collaboratively, using a simple markup language and a web browser. A single page in a Wiki website is referred to as a "Wiki page", while the entire collection of pages, which are usually well interconnected by hyperlinks, is "the Wiki". Wiki is essentially a database for creating, browsing, and searching through information [13]. Wiki engines enable easy creation of links between terms, pages and titles, enlarging in another dimension of knowledge sharing [12].

Considering Wiki's capabilities, it is used as the main body part of the EEKC for storing and sharing information and knowledge.

\subsection{Semantic Wiki}

A semantic Wiki is a Wiki engine that uses technologies from the semantic Web to embed formalized knowledge, content, structures and links, in the Wiki pages. Formalized knowledge is represented using semantic web frameworks and is thus accessible and reusable by web applications. Within the Wiki, this knowledge can be used to propose enhanced features such as better document searching, suggesting new links, identifying acquaintance networks, dynamic content update, checking and notification, etc [14]. 


\subsection{Summary}

Knowledge, especially tacit knowledge, needs to be modeled using automatic methods and then should be documented to enter into the EEKC. This starts the development of the EEKC and after a while the EEKC users would be able to add, review, and update the knowledge. To extract and model the knowledge in work processes, some protocols to use the EEKC by the IAC members need to be designed. Knowledge and information should also be evaluated and validated to ensure they have reliable quality to use in assessment projects.

Web 2.0 tools and particularly Wiki pages are used to design the EEKC. Using the Web 2.0 tools significantly enables the IAC members to contribute in continuous improvement process. Security levels are defined and are completely under the control of system administrator. All documents and files can be archived and get versioned. They can get user comments, and when they are finalized and validated, they would be archived in the document banks. This would be truly helpful to improve the current processes at the IAC. All members can upload, download and share the last version of each $\mathrm{AR}$ in a unique and dynamic place.

Once information and knowledge are saved and archived under a respective knowledge domain, the IAC directors would be able to monitor the system. They can find the strengths and weaknesses in each knowledge domain and identify goals for knowledge creation or development. This significantly helps them design improvement plans to enhance the system. 


\section{CHAPTER 3: THE IAC WORK PROCESSES, AND RELATED A P P L ICA T IONS IN EEKC}

The main step to design and develop the EEKC is to understand the work processes at the IAC. The IAC work is divided into three main phases including pre-assessment, assessment, and post-assessment. These three phases are shortly reviewed in the introduction Section. In this Section, the phases are studied based on information and knowledge flow. Each phase includes various major processes which are noted in Sections 3.1, 3.2, and 3.3. Should there be a possible EEKC application for each process, it is explained.

The objective of analyzing and understanding the IAC work processes is to:

- Define how the EEKC can support the IAC processes in various activities,

- Define the necessary systems and sub-systems of the EEKC,

- Obtain and share hands-on experience and knowledge that enable the IAC to enhance its tasks,

- Manage documents and information in the three phases,

- Capture and share knowledge from documents and information.

\subsection{Pre-assessment Processes}

a) Process: (a) Engage the plant personnel and utility company personnel in discussions on conference telephone calls to discuss the manufacturing process and key energy utilization indicators. (b) Discuss the operational characteristics of energy systems as well as the EEM already implemented and those that are being considered to have good energy saving potential. 
EEKC Application: This process helps the IAC members catch general information about the manufacturing processes at the plant. Information gathered during this process would be the first entry to the EEKC which initiates the energy profile for the company. The energy profile is created in this step and would be completed in the following steps.

b) Process: Perform a QuickPEP analysis to identify potential EEM and enhance the energy profile for the plant.

Supplement Information: The QuickPEP is software tool provided by the U.S. Department of Energy to help industries in the United States identify how energy is being purchased and consumed at their plant and also identify potential energy and cost savings. QuickPEP is designed so that the user can complete a plant profile in about an hour [15].

EEKC Application: The QuickPEP output provides summarized information to save energy designated based on current situations at the plant. There is a specific section in the EEKC for the QuickPEP results providing solutions to benchmark and compare the savings with other companies' results. Figures and tables developed by the QuickPEP should be graphically presented and archived in the EEKC.

c) Process: Using the IAC database to find and study the same type of manufactures.

Supplement Information: IAC Database is a collection of all the publicly available assessment and recommendation data. This includes information on the type of facility assessed (size, industry, energy usage, etc.) and details of resulting recommendations 
(type, energy, and dollars savings etc.) [16]. As of 07-20-2011, the IAC database contains [16]:

- 15,050 Assessments

- 112,836 Recommendations

EEKC Application: Query the IAC database on potential EEM based on the manufacturing characteristics of the plant. Using the IAC database and similar ARs for different manufactures helps the IAC members catch ideas and information to develop each AR. They have access to all versions of an AR implemented in the past. In this process, some valuable knowledge may be created/exchanged combining different versions of an AR. This knowledge needs to be entered in the specific Wiki page for each particular AR.

d) Process: Benchmark the energy usages of the manufacturing facility with respect to similar facilities that have had an IAC assessment from the WVU IAC in the past.

EEKC Application: There are usually a number of energy assessment reports available for plants with similar manufacturing process. Using the previous reports would prepare useful information to benchmark the energy usage of the plant.

Studying energy usages of the similar facilities and comparing them to the theoretical values may help the IAC members find the practical energy usage baseline for different machines and processes. Simply stated, any energy usage above the energy usage baseline could be a waste and an opportunity to save energy. There needs to be a specific section in the EEKC to benchmark energy usage and archive the results using 
figures and spreadsheets. This section is connected to the IAC database and the AR archives.

e) Process: Identify potential "gaps" in energy efficiency based on preliminary analysis of the data obtained from the plant.

Supplement Information: The detailed analysis of the utility bills is an important step towards this process. The utility bills show the energy consumption with respect to time and season and may reveal energy consumption patterns that require attention in terms of electricity, natural gas, and other fuels.

EEKC Application: Once the energy usage baselines are provided, analysis of data obtained from the plant in the EEKC spreadsheets makes it more convenient and efficient to identify potential gaps in energy efficiency comparing the current usages with efficient usages.

These gaps are currently identified, but they are only used to develop the ARs. In the EEKC, all of these gaps are archived in forms of data, text, and figures. This archive provides the ability to focus more on the reasons and root causes of the gaps. The EEKC make it possible to use professional tools for data analysis, i.e. Data Mining. Hence, it could become an assistant decision maker not only for the IAC members but also for higher levels of decision makers.

f) Process: Identify existing rebates and incentives offered in the state as well as from the utility companies. 
EEKC Application: There are many different resources for rebates and incentives. The IAC members usually spend a considerable time to find the offered programs in the state. A section in the EEKC for the rebate and incentive programs is created which archives and gathered all programs offered for different manufactures. This section initiates with the current information and becomes more developed by the IAC members over time. The EEKC would provide up to dated information for different manufacturers in the region and helps the IAC save time in this process and obtain more reliable (and validated) results. EEKC Members can comment on each program and make it more practical.

g) Process: Review DOE ITP Best Practices fact sheets for possible application in development of EEM as well as suitability of commercialized energy efficient technologies based on preliminary analysis of opportunities at the plant.

EEKC Application: The DOE ITP Best Practices fact sheets are gathered and entered in the respective sub-systems in the EEKC. These pages are linked to the relative ARs.

\subsection{Assessment Processes}

a) Process: Make a presentation of the preliminary findings from the pre-assessment phase and discuss potential "gaps" in energy efficiency with plant personnel.

EEKC Application: The results of pre-assuagement activates along with the energy profile of the company are available in the EEKC. This helps the IAC members prepare the presentation files. The final presentation files are documented in the pre-assessment 
section at the EEKC. At the end of the assessment project, the proposed energy usages are linked to the results of energy usages before the assessment.

b) Process: Discussion with plant personnel on the characteristics of the manufacturing process and the energy utilization aspects on major energy systems.

Supplement Information: The discussion clarifies any missing details in terms of process equipment, adopted technologies for operating the process and energy consuming equipment, and the specific methodologies being used in linking the process equipment with other energy consuming equipment.

EEKC Application: To make sure that the IAC members discuss with plant personnel on all necessary characteristics of the manufacturing process and the energy utilization aspects which are going to be used in development of ARs, an assessment tool case needs to be created and presented in the EEKC. This tool case includes the checklist and essential questions created over a number of assessment projects for major energy systems.

For each particular AR, the required information may change if the manufacturing parameters are different, which might be difficult for non-experienced members to catch all essential information. Hence, the assessment tool case would be an assistant for the IAC members.

c) Process: Discussion with plant personnel about operational constraints and barriers to implementation of EEM in specific energy systems with respect to factors such as the integrity of the manufacturing process, product quality, and worker safety and health. 
d) Process: Plant tour and observation of the manufacturing process and operation of the energy systems.

Supplement Information: The plant tour is an important step towards the identification of EEM. The interaction with plant personnel during the tour often reveals interesting operational characteristics of the energy consuming and process equipment. It also facilitates discussion of any EEM that have been attempted in the past by the manufacturing facility.

Following the discussion of plant operation and existing concerns, the audit team and one or more plant representatives tours the plant and conducts a walk-through audit to understand the operations performed at the plant, and to determine the flow of materials from input or receiving, through processing, and finally to shipping. One audit team member is responsible for taking notes on the manufacturing processes and operations, and another compiles an inventory of major energy using equipment. If the equipment inventory is obtained in advance, then the job is to confirm the list of large energy consuming items [19].

The plant tour is the point at which the experience of the professional staff of the IAC - the Director and/or the Assistant Director - makes a major impact on the quality of the results. Knowledge of manufacturing processes, equipment, and operating procedures is necessary to spot the areas where energy conservation and energy efficiency improvements can be made. Inefficient process heating equipment, low-efficiency motors, large quantities of wasted heat, poor match of fuel forms to process needs, poor process or personnel scheduling, and inefficient scheduling of large electrical loads are 
only some of the areas where highly experienced personnel can make significant differences in getting good AR's and alternatives identified [19].

e) Process: After the planning meeting within the assessment team, intensive data collection efforts, observations, and interaction with plant personnel follow.

Supplement Information: The data measurement process is critical, as effective control cannot be achieved without effective measurement. Followings are some example of data collection activities during the assessment [19]:

- Combustion efficiency is measured for all major fuel-burning equipment. At the minimum, excess oxygen and stack temperature are measured in oil and gas boilers. Carbon monoxide and carbon dioxide levels are also measured if equipment is available.

- Efficiency and power factors of large motors and motor-driven equipment are measured when there is reasonable and safe access to power conductors. Singlephase or three-phase measurements are taken as appropriate to obtain the efficiencies and power factors. Similar measurements are taken when safe and practical for all other large electrically powered equipment. Examples of such equipment include arc furnaces, electrolytic cells, and infrared or induction heaters.

- Other factors that affect energy consumption are measured including process temperatures, equipment temperatures, and working temperatures in various plant and office areas. Light levels are measured in most areas of the plant and offices. 
Air flow and ambient temperature are measured to determine efficiencies of HVAC systems and duct losses throughout the plant.

EEKC Application: In the energy profile for the plant, there is a data bank which includes all data gathered during the assessment, i.e. electric current and air pressure obtained using data loggers. This bank is accessible to the EEKC users with respect to security levels, and if it is needed, plant personnel are able to update the data in a newer version.

f) Process: Discussion of collected data with plant personnel.

Supplement information: The data collected will be subject to a preliminary analysis and validation at the site to discuss any potential anomalies that can be best explained only by the company personnel.

The techniques that the IAC uses in generating energy savings based on assessments vary, but share a general theme with respect to effectively recognizing the potential for large energy savings in the various energy systems. In other words, there may be numerous ideas to save energy within a manufacturing facility; however, many of these opportunities may not generate much energy savings and may also have poor payback on investment. Since the energy assessment is usually done within one or two days, the IAC do not spend much time on such opportunities because of the possibility of not having enough time for data collection and analysis of some of the larger energy savings measures with an attractive rate of return. 
g) Process: Intensive discussions on implementation potential of EEM with plant personnel and obtaining preliminary non-binding verbal agreement from them on interest in implementing EEM.

h) Process: Discussion with plant personnel on financial hurdle rate for implementation of EEM as well as other barriers for implementation.

i) Process: Expose plant personnel to DOE ITP services and commercialized energy efficient technologies that may apply and discuss energy efficient technology "gaps" that may of interest.

\subsection{Post-assessment Processes}

a) Process: Development of AR based on EEM.

Supplement information: The assessment recommendations developed for the manufacturing facilities, using a process of data logging, monitoring, reference to best practices knowledge, interaction with company personnel, and solid engineering principles, will have a strong potential for implementation. The report produced consists of: (1) the data and other information derived from records and measurements made during the assessment; and (2) the specific recommendations, together with the assumptions used and calculations made.

EEKC Application: An important objective of the EEKC is how it presents the saving opportunities for the company. The final report and full text of each AR are stored in the EEKC, although the objective is not only to show the entire report, but also to present the 
practical information and knowledge extracted from each particular AR. This may be initiated by summarizing and enhancing each AR considering the EEKC objectives.

Another notable tool in the EEKC is archive of all versions of a particular AR. Since improvement in ARs is a continuous activity, having access to all previous versions and the last edited version (and more importantly the validated version) would help the IAC members save time on preparing reliable results, increase the quality level of work, and follow the comments generated on the previous versions.

b) Process: Work with plant approved vendors to refine AR as well as to identify the need for additional data.

c) Process: Based on discussion with vendors, identify operational specifications for any suggested energy efficient equipment in AR.

d) Process: Determine the robustness of AR based on variations in product, process, and system parameters, as identified by the plant personnel.

e) Process: Determine and apply any available rebates and incentives to the AR.

EEKC application: The rebate and incentive part in the EEKC, presented in Section 3.1.f, is used for this process. It needs to be updated after the assessment and post-assessment phases.

f) Process: Send draft report to the plant personnel. 
Supplement information: The draft report includes a detailed description of all manufacturing operations, a plant layout sketch and description, a major equipment list, and an analysis of how energy is used throughout the plant. Each of the energy, waste, and productivity recommendations includes estimates of energy conserved, costs saved, implementation costs, and simple payback periods.

g) Process: Discussion on the report with plant personnel through conference calls. Supplement information: Schedule the technical assistance activity and presentation after obtaining assurance that at least one key decision maker in terms of upper management personnel is at the meeting.

h) Process: During the technical assistance activity and presentation, engage the plant personnel on discussions on implementation and identify clearly what would be the next steps and how the IAC can assist in implementation.

EEKC application: The information gathered in this process is truly valuable. The hidden reasons that an energy saving idea cannot be implemented or should be implemented are identified in this process. These reasons would prepare great knowledge and should be carefully extracted during the sessions with the plant personnel. The obtained knowledge then enters to the respective section of the EEKC. This knowledge gives insight to the IAC members to improve and enhance the ARs for that plant as well as for the prospective assessment projects.

i) Process: Additional data collection, if needed, is done during the TA visit. 
j) Process: Train the plant personnel on the use of DOE ITP Best Practices software tools using the data collected during the assessment process.

Supplement information: The plant personnel who review the report will see the use of DOE's Office of EERE/ITP Best Practices software incorporated into the generation of many of the energy efficiency recommendations, using the data collected at the plant. This will pave the way for the company personnel to have the interest and motivation to obtain training in some of the Best Practices software and utilize them in the continuous energy efficiency process at their facility.

k) Process: Ensure that the plant personnel know that the IAC is available for continuous interaction for purposes of enhancing the potential for implementation of AR through the SEN project.

EEKC application: The plant personnel should have access to the EEKC with respect to the security levels. They can obtain baseline energy usage for their specific energy systems as well as practical tips to save energy. They may also comment on their ARs which could provide their feedback to the IAC members.

1) Process: Update draft report and finalize and send it to the plant personnel.

Supplement information: The final report contains the following:

a) Executive summary of energy savings, cost savings, implementation costs, and payback on investment.

b) Classification of the assessment recommendations into low, medium, and high, in terms of payback on investment for easy review by management. 
c) The energy billing summary for electricity, natural gas, and other fuels showing the rates to be used in the energy savings calculation.

d) The assessment recommendations, each detailing the energy savings summary at the very beginning, along with the description of the recommendation, technical formulae used in calculations, assumptions, if applicable, and implementation aspects. The bottom line in terms of payback on investment is shown for each recommendation, along with the annual cost savings, and $\mathrm{CO}_{2}$ emissions saved.

e) Digital pictures of the equipment and specific areas to illustrate the nature of energy saving recommendations, subject to company approval for taking the pictures.

f) The use of DOE Best Practices software tools integrated into the development of appropriate recommendations.

m) Process: Capture the company approved knowledge from the pre-assessment, assessment, and post-assessment phases for representation on the EEKC for purposes of replication of energy savings and enhancing the potential for widespread dissemination of knowledge on energy efficiency.

\subsection{Summary}

The main three phases of SEN assessments and their processes were studied in this chapter. Also for each process, possible applications were discussed to be effectively built into the EEKC. The designed system along with usage protocols are presented in the rest of this thesis. 


\section{CHAPTER 4: DESIGN AND DEVELOPMENT OF} THE E E K C

\subsection{EEKC Architecture}

EEKC architecture ${ }^{1}$ determines how the information and knowledge in the EEKC its Web pages, documents, lists, and spreadsheets - are organized and presented to the users.

\subsubsection{Questions to Ask for Designing the EEKC}

Questions to ask for designing the EEKC are as follows:

- How will the EEKC be structured and divided into different sub-systems?

- How will data, information, and knowledge be presented?

- How will site users navigate in the EEKC?

- Is there any specific content which needs to be included or excluded?

- What types of content will live on the EEKC site?

- How will content be tagged and how will metadata be managed?

- Does any of the content on the EEKC site have unique security needs?

- How will information and knowledge be targeted at specific audiences?

These questions are considered for designing the EEKC.

\subsubsection{Three High Priority Factors in EEKC Architecture}

For energy efficiency content in the EEKC, the factors in Figure 3 should be considered. Determining the priority of the factors for each type of content is critical.

\footnotetext{
${ }^{1}$ Microsoft Governance report for SharePoint Server 2010 is used as a platform to develop the EEKC architecture [17].
} 


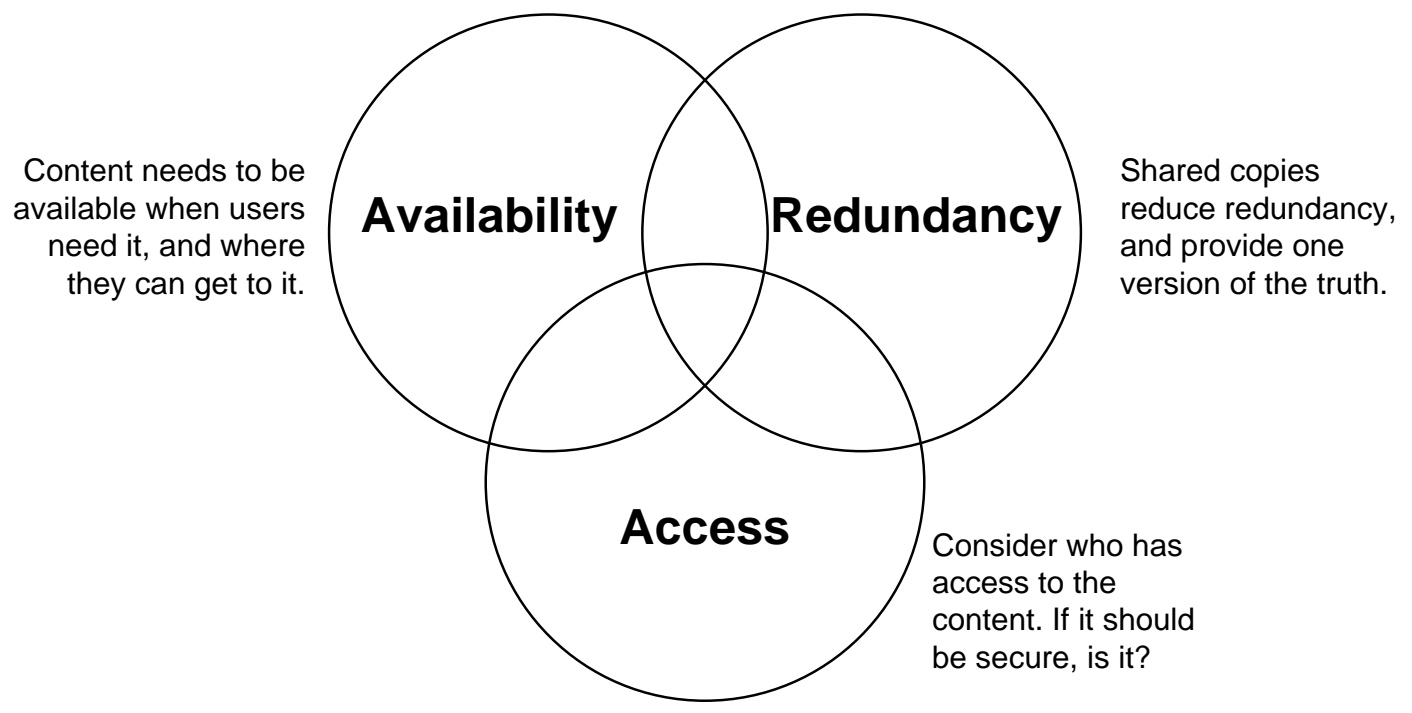

Figure 3: Three important factors to be considered in the EEKC architecture [17]

For example, having a single copy of a document is good for reducing redundancy, but it is a problem for availability and access if it is deleted. For the EEKC, all of the three factors in Figure 3 are assumed to be high priority for consideration.

\subsubsection{Information Governance}

The energy efficiency content needs to be governed using tools for content management, including:

- Use workflows and approval for document centers and site pages, wherever official documentation is stored.

- Use approval for published web sites to control pages.

- Use version history and version control to maintain a history and master document.

- Use content types with auditing and expiration for document libraries to manage document lifecycle. 
- Identify important corporate assets and any sites that contain identifiable information.

\subsection{Information Flow in IAC}

Figure 4 represents the information flow in the IAC work processes.

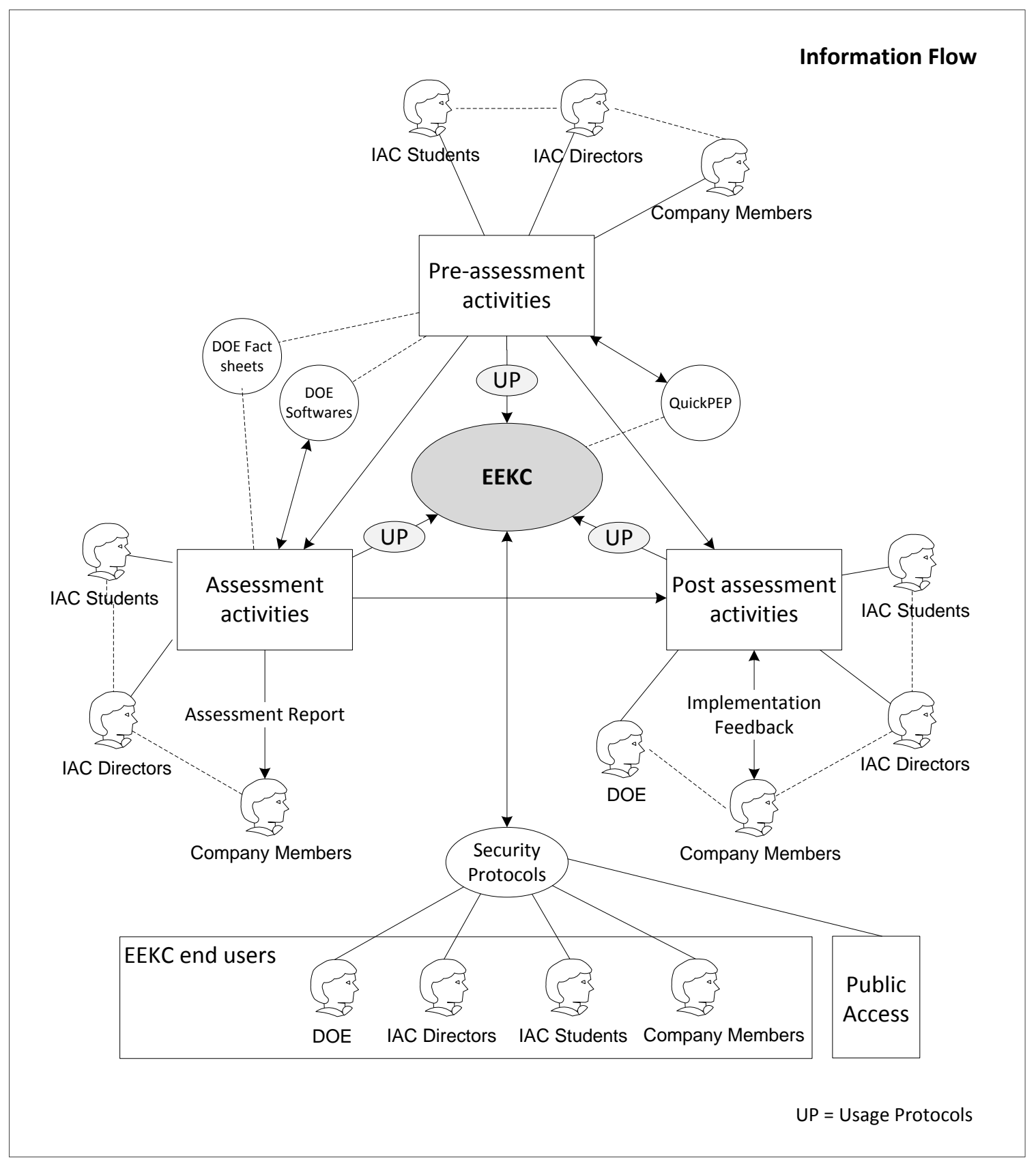

Figure 4: Information flow in IAC 


\subsection{Steps to Design the EEKC}

The first step to design the EEKC is to prepare a powerful server and a reliable database configurable with other databases. Figure 5 simply illustrates the steps to prepare the main infrastructure to build for the EEKC.

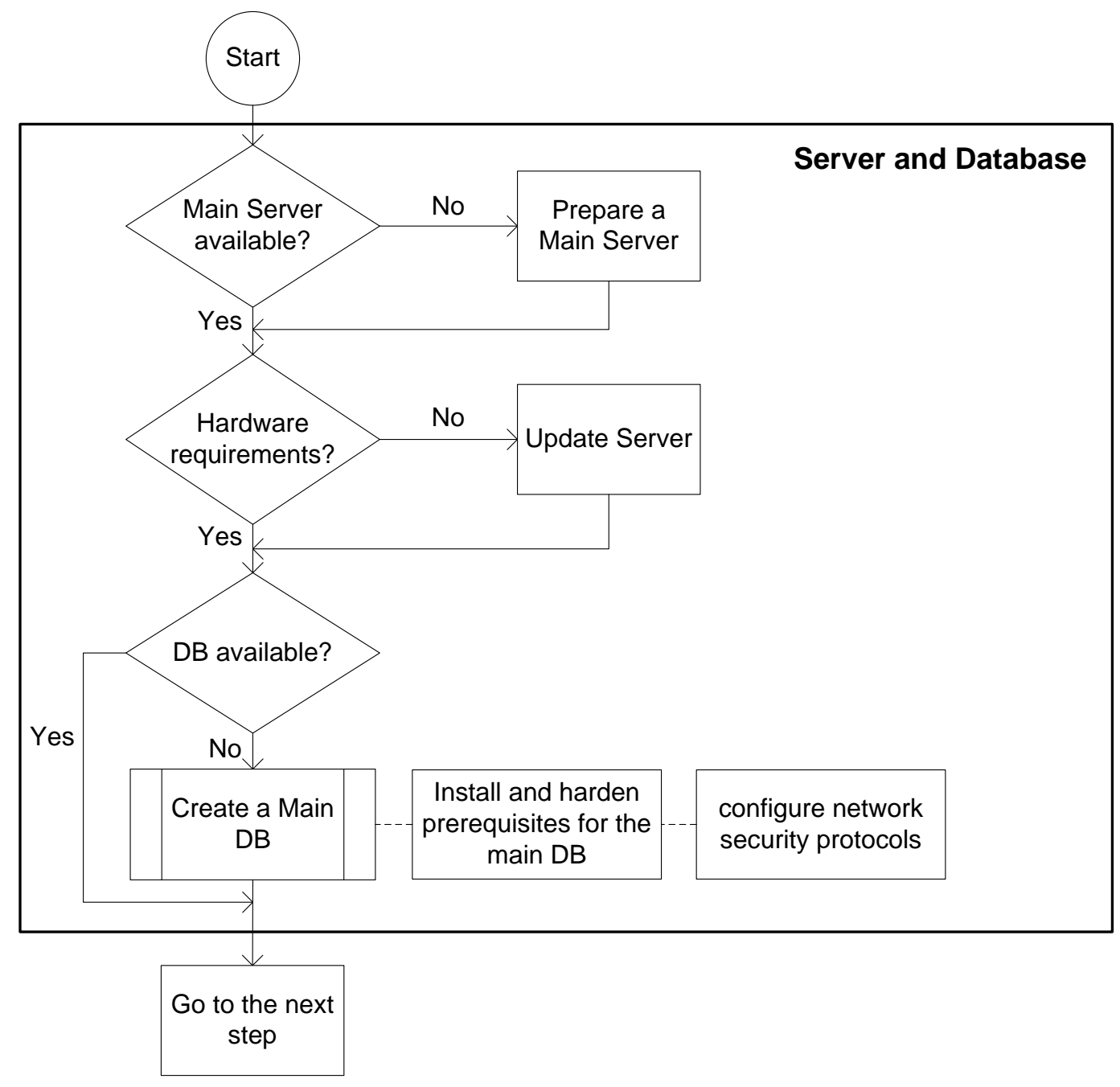

Figure 5: Steps to prepare the server and database

Once the server and database are ready, the next step is to develop the web tools.

Figure 6 shows the processes to prepare the web tools. The last step would be 
enhancement and development of the EEKC with extracted information and knowledge.

Figure 7 presents the processes for this step.

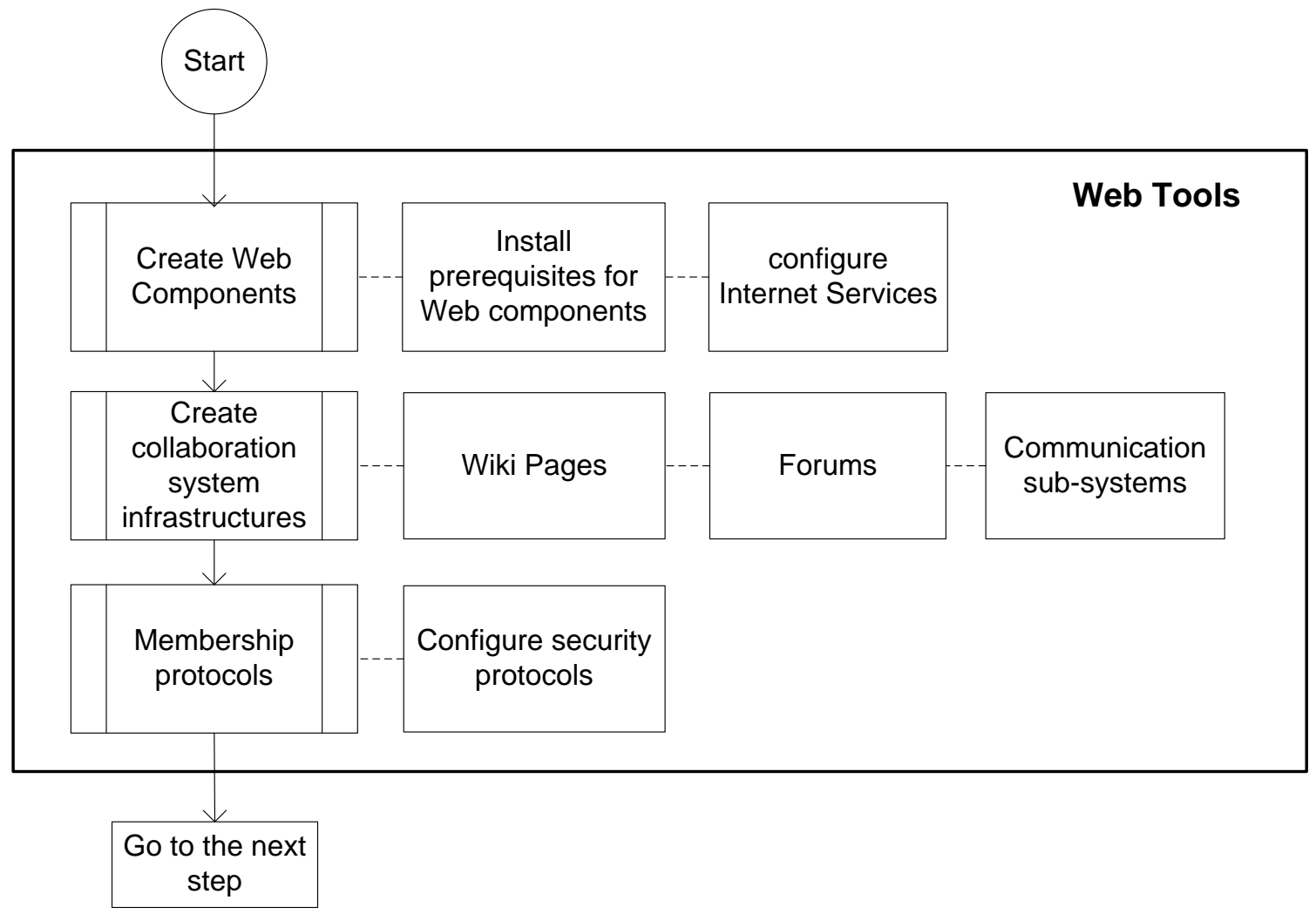

Figure 6: Steps to prepare the web tools

After the three steps are done and membership protocols are defined, the IAC members can join and contribute to enhance the system. The next step to develop the EEKC is to develop protocols to be used by the IAC members in their work processes. 


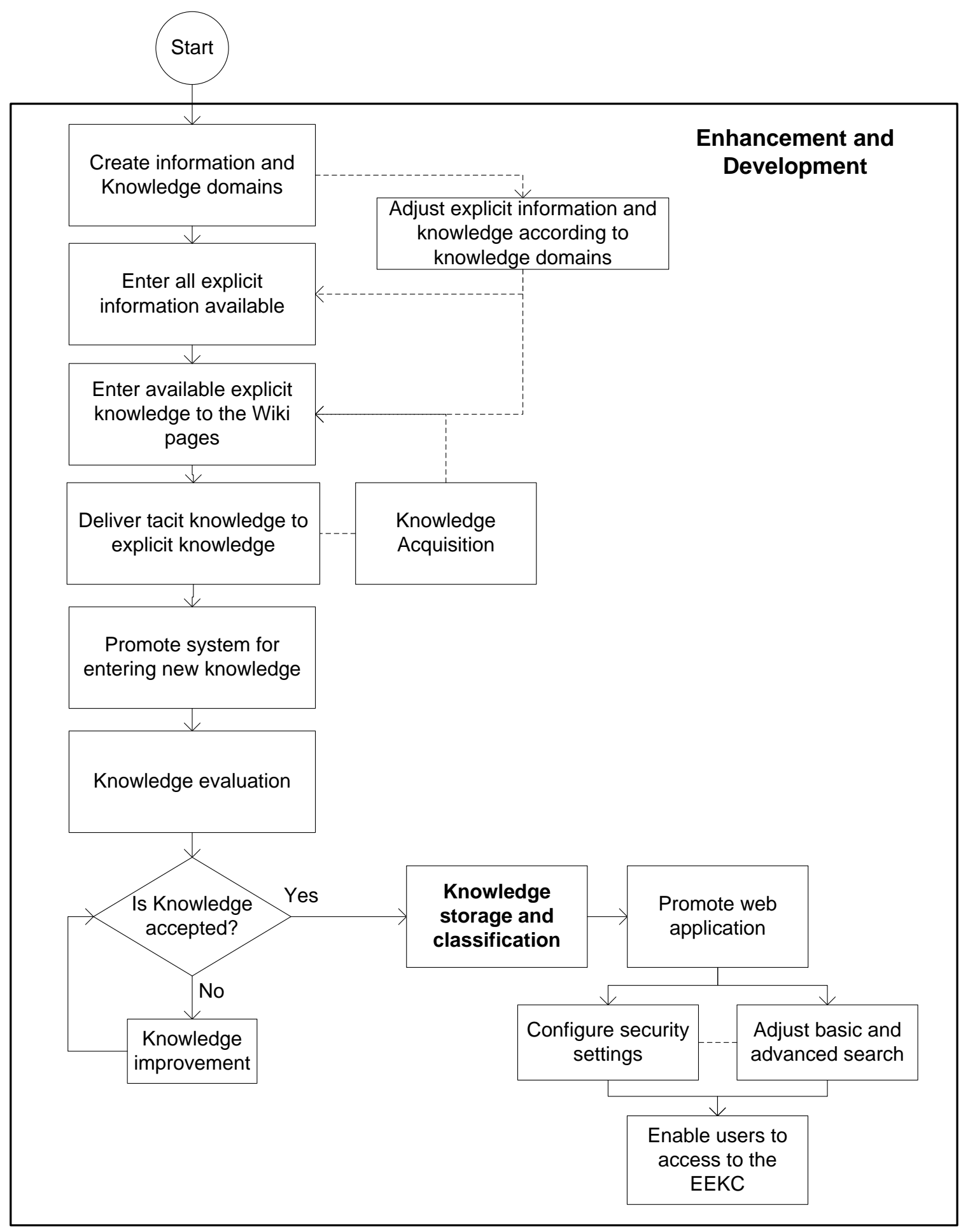

Figure 7: Enhancement and development steps 
An attempt was made to establish a server and database for the EEKC, but establishing and maintaining a server need a huge amount of resources. Therefore, a built-in server in a third-party company (Google) is used in which high security technologies are used.

\subsection{EEKC Pages}

The EEKC is designed based on the applications discussed in Chapter 3 and characteristics presented in this chapter. An SEN assessment is then used to start developing the EEKC for which the results are presented in Section 4.7.

All pages in the EEKC are created using Wiki technology that are easily customizable. The first page of the EEKC illustrated in Figure 8 is public and anyone with its web address can see the pages. To get into the private pages, users are required to have access; otherwise they would not be able to see the page. More details about permissions and security levels are presented in Section 4.8.

Main sections of the EEKC are shown in site navigator, the left side in Figure 8. One of these sections is named SEN Assessment under which client profiles are created. The first layer of the client profile is public and lower layers such as pages for AR bank and vendor information are private. Another main section named Knowledge Center has different sub-pages for major equipment such as air compressors, motors, boilers, etc. Each major equipment type has also sub-pages for different kind of ARs in which AR file, Wiki (from now on, the page designed for saving and sharing knowledge is called as Wiki), vendor information and rebates and incentives are available. 


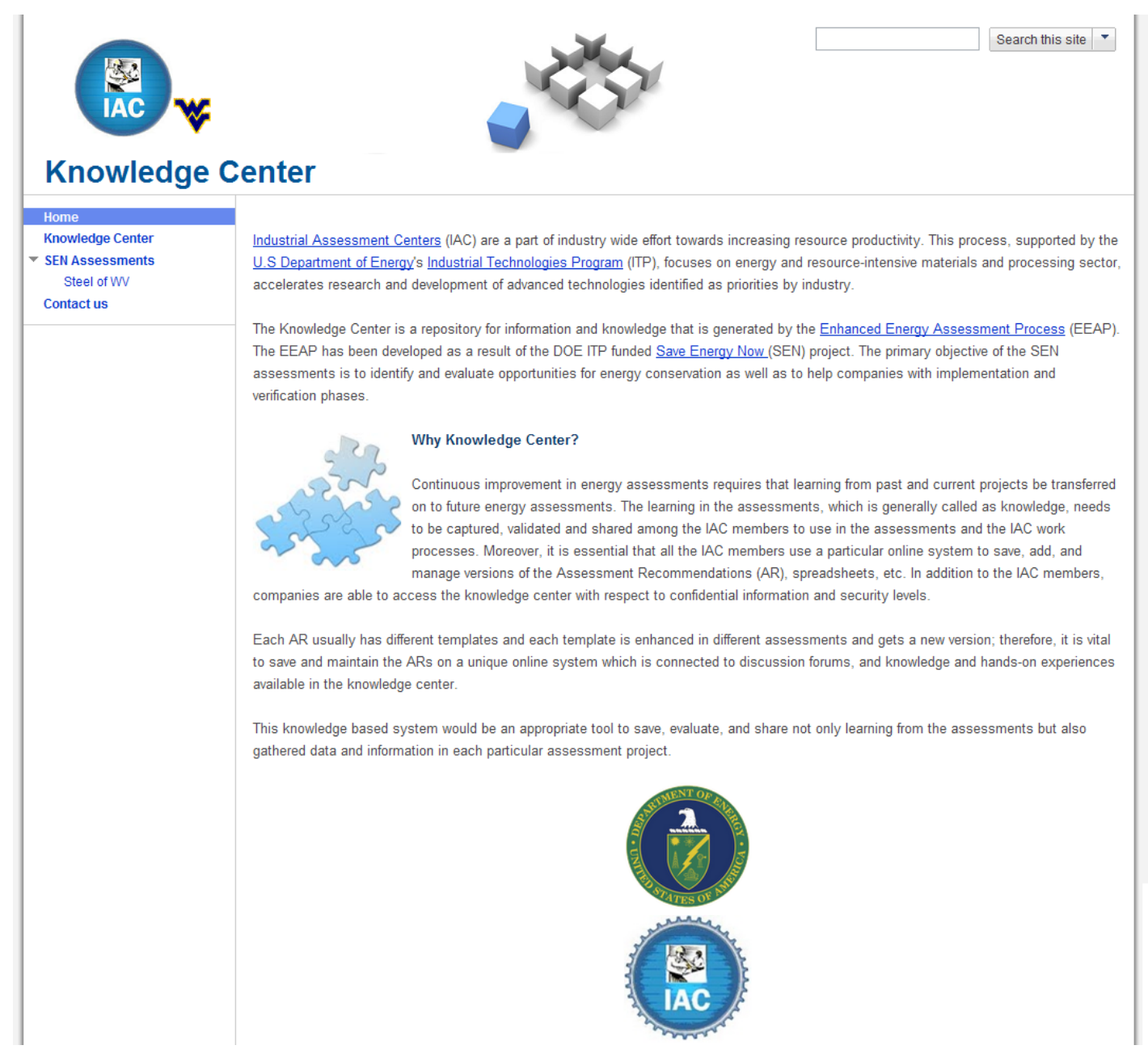

Figure 8: First page interface

\subsection{Usage Protocols}

The output of this study is not only to design a knowledge center but also is to develop protocols to use the EEKC in energy efficiency assessments. Three protocols are designed to use in each of pre-assessment, assessment, and post-assessment phases. The steps of these protocols are designed according to section three, study of the IAC work processes and related application in the EEKC. The EEKC users are urged to follow these protocols in their work activities. The pre-assessment protocol is shown in Figure 9. 


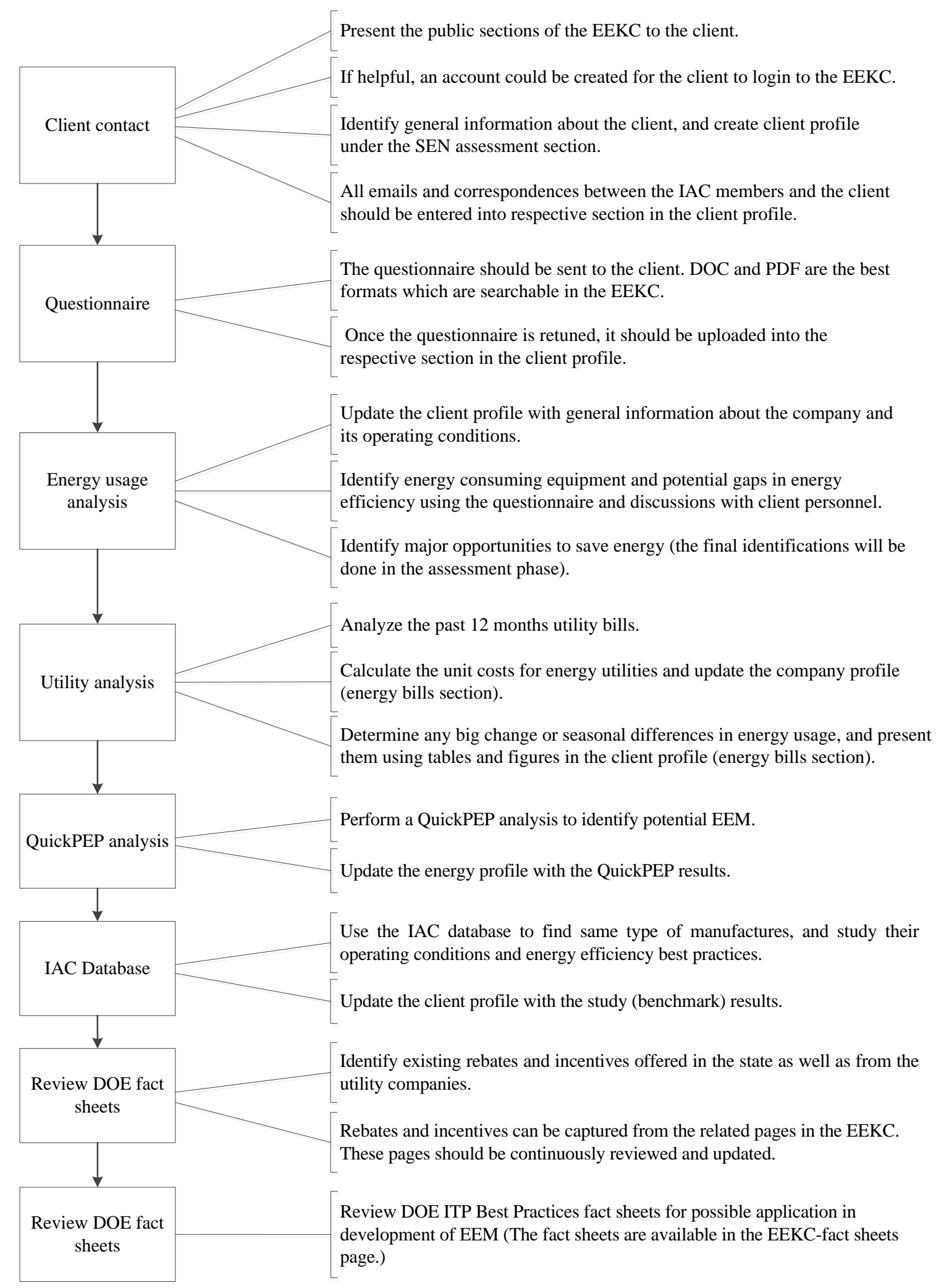

Figure 9: Pre-assessment protocol 
It is essential that all the steps in in the pre-assessment protocol will be followed up; otherwise the EEKC would not be effectively used. The key to make the EEKC useful is to use it in assessments. Hence, using this system should be seriously considered among the first priority tasks at the IAC.

After the pre-assessment protocol is thoroughly done, assessment protocol should be followed. However, the assessment is walk-through the plant and so there are not many things to do in the EEKC during the assessment. The assessment protocol is illustrated in Figure 10.

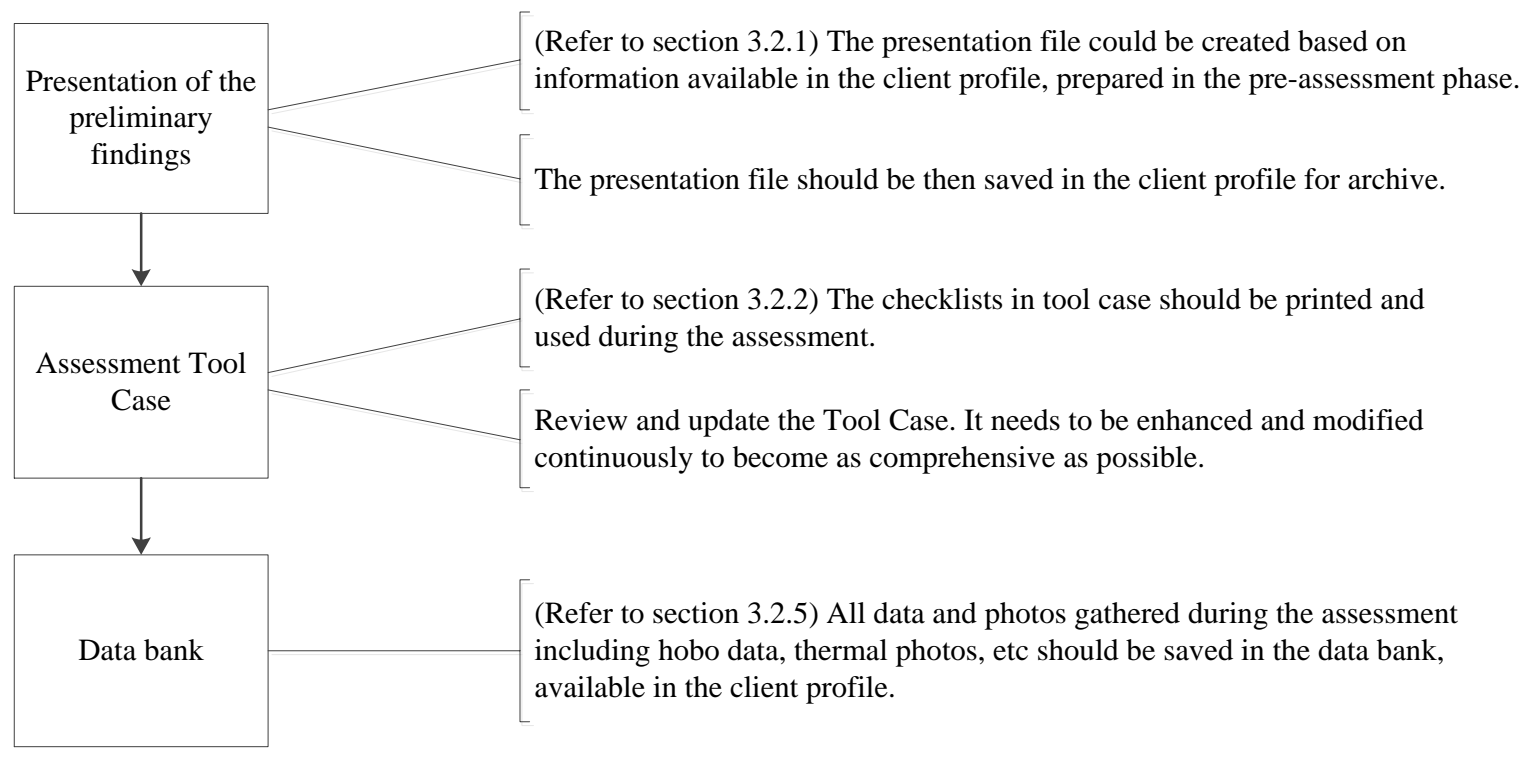

Figure 10: Assessment protocol

The next and last main phase in energy assessment is post-assessment for which the protocol is shown in Figure 11. 


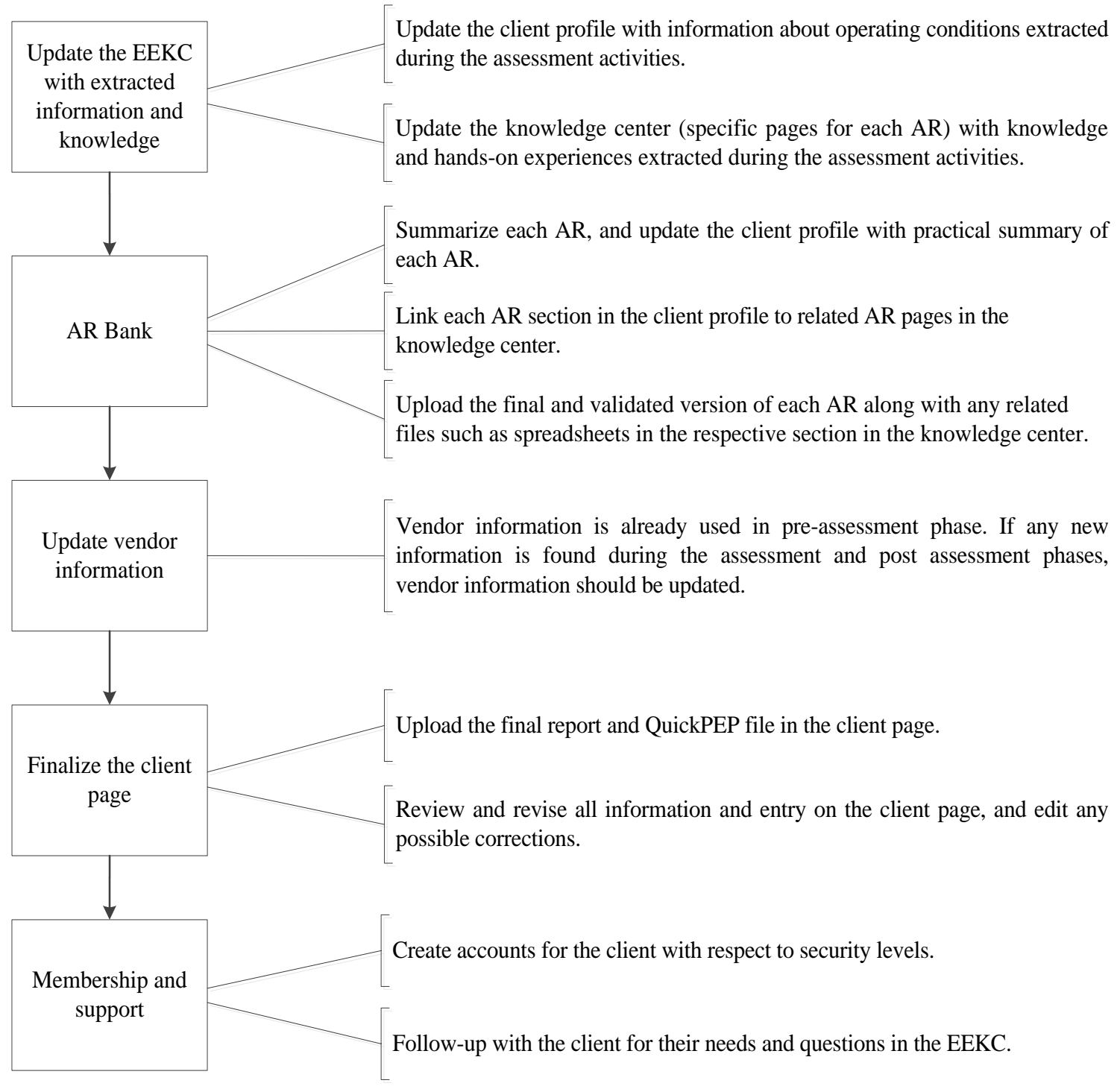

Figure 11: Post-assessment protocol

The protocols presented above should be reviewed after some assessments and then they need to be updated to catch proper results. In order to improve the protocols, IAC members' feedbacks are needed. They are able to send their feedback through the system. 


\subsection{Company Profile}

The table of content of the company profile is shown in Figure 12. Contents are made up with information and knowledge extracted from the complete report. To develop a new profile, the first step is to create a new page for the respective company. A company profile can be created as follows:

- First, log in to the system using the administrator account.

- Click "Create page".

- Select the template to use, "SEN projects".

- Enter a name for the page. This name is used as the page title and its link title. Generally, end users will see this name at the top of the page.

- $\quad$ Put the page under the proper sub-address. For the SEN projects, click "Choose a different location" and then click "SEN Assessment".

After doing the steps above, the necessary parts for the company profile should be created. This company profile could be simply copied to a new page and customized for future projects. To do so, on the start page of the new page, click "Edit page" and paste the copied content. Once the page is created and filled with the assessment report, each AR needs to be linked to the related page in the Knowledge Center. As it was mentioned earlier, the page under the SEN assessment address would be public, but the pages in the Knowledge Center section are secured. 


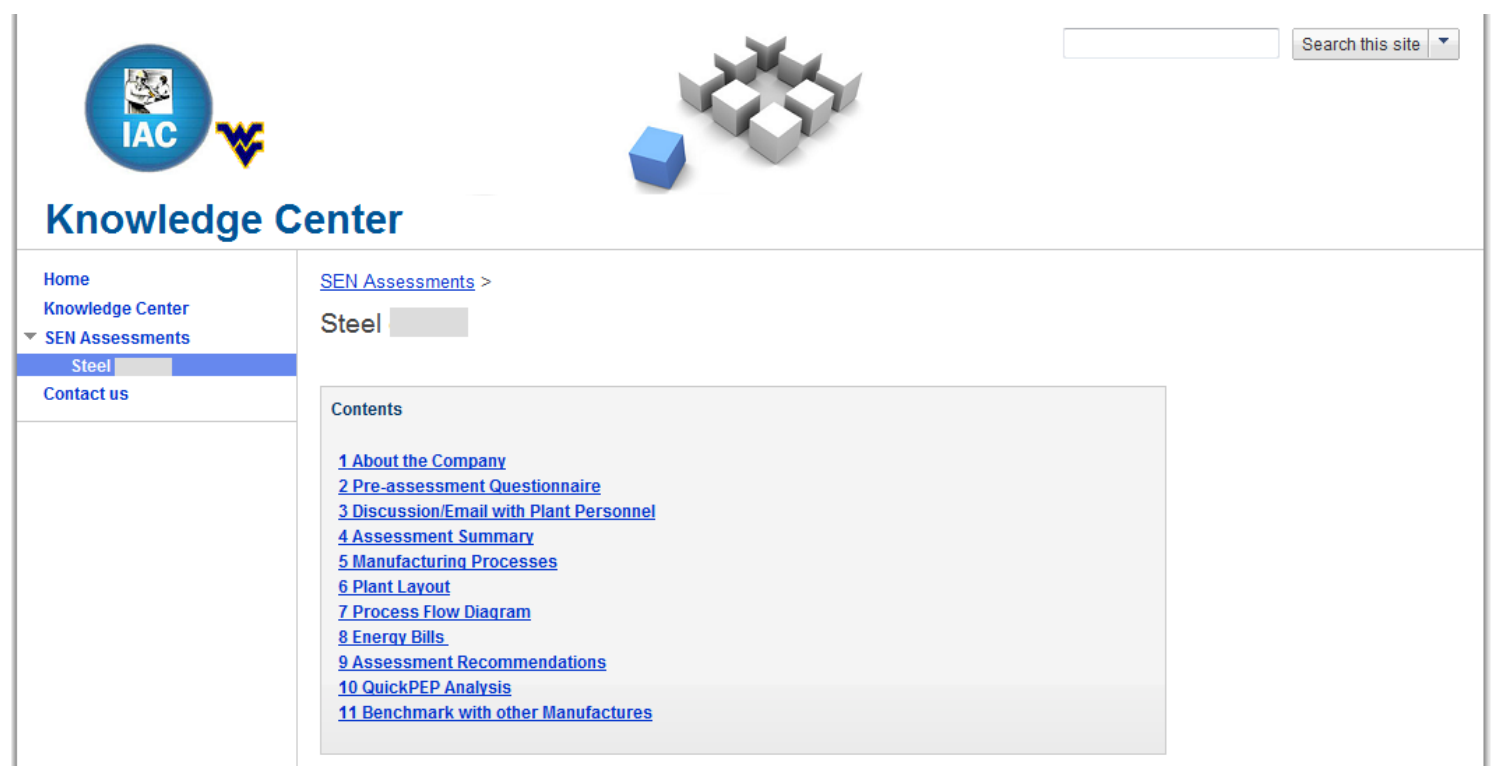

Figure 12: Table of content for a company profile

The company profile is a page to show the practical results summarized out of the complete report (complete reports have usually more than 80 pages). It is a quick way to look at the entire report with ability to see more details along with knowledge and hands-on experience for each particular AR in links and sub-pages.

\subsection{Case Study, Company X}

The protocols are followed up for the selected SEN assessment (Company X) and all required parts for the EEKC are created. Extracted knowledge and information are then entered into the EEKC. The pages and sub-pages for the company $\mathrm{X}$ in EEKC are shown in Figure 13. 


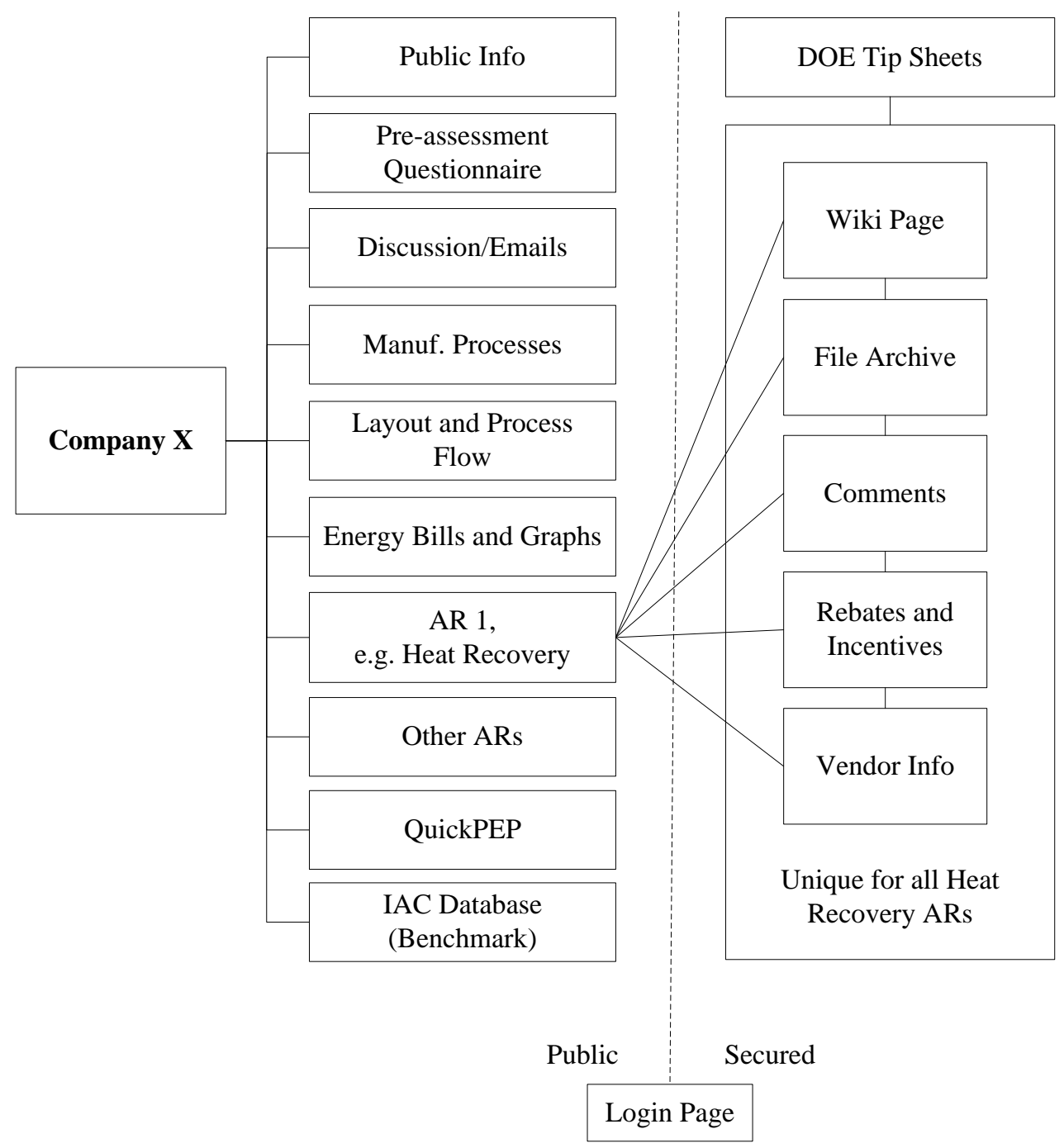

Figure 13: Profile and related pages for company $\mathrm{X}$

Pages and sub-pages presented in Figure 13 along with explanations to prepare their required contents are presented in following sections.

\subsubsection{Pre-assessment}

a) Public information: Public information about the company $X$ is found from their website and other resources on the Internet. Considering confidentially agreements, 
information such as company name, contact information, web address, and plant and product specifications can be uploaded into the public information section in the company profile.

\section{b) Pre-assessment Questionnaire:}

The questionnaire filled by company personnel needs to be uploaded into the respective section in the company profile. Besides the link to download the questionnaire, important information extracted from the questionnaire should be also presented into the profile page as shown in Table 3.

Table 3: Information extracted from the questionnaire

\begin{tabular}{|l|l|}
\hline Plant operating hours & $(7: 30 \mathrm{am}-4: 00 \mathrm{pm}) \times$. days/week x 52 weeks/year \\
\hline Office hours & $24 \mathrm{hrs} /$ day x 7 days/week x 51 weeks/year \\
\hline Number of employees & 339 \\
\hline Number of salaried employees & 79 \\
\hline Plant and office area & $704,283 \mathrm{ft}^{2}$ \\
\hline Annual production volume & 250,000 tons \\
\hline Labor rate for maintenance & $\$ 46 / \mathrm{hr}$ \\
\hline Major energy consumers & Electronic Furnaces, 2 units of 65 Tons \\
\hline
\end{tabular}

More importantly, information about major consuming equipment could be noted into the company profile. 


\section{c) Discussion/emails:}

Discussion and emails with company personnel include important information which should be extracted to use in developing ARs. For instance, it was found that which ARs from the past assessment in 2003 for company X were not implemented and what were the causes as shown in Table 4.

Table 4: Status of the previous ARs for company X, extracted from emails/discussion

\begin{tabular}{|c|c|c|}
\hline AR No. & $\begin{array}{c}\text { Implementation } \\
\text { Status }\end{array}$ & Causes $^{2}$ \\
\hline 1 & Not implemented & C1 \\
\hline 2 & Not implemented & C2, C3 \\
\hline 3 & Implemented & - \\
\hline 4 & Implemented & - \\
\hline 5 & Implemented & - \\
\hline 6 & Implemented & - \\
\hline 7 & Implemented & C4 \\
\hline 8 & Not implemented & C5, C6 \\
\hline 9 & Not implemented & - \\
\hline 10 & Implemented & - \\
\hline 11 & Implemented & - \\
\hline 12 & Implemented & \\
\hline
\end{tabular}

Information extracted from the discussion/emails with company personnel should be presented in the respective section in the company profile.

\footnotetext{
${ }^{2}$ Due to confidentiality reasons, the causes cannot be mentioned in this thesis.
} 


\section{d) Manufacturing processes:}

Manufacturing processes of the company X shown in Figure 14 is uploaded in the company X's profile.

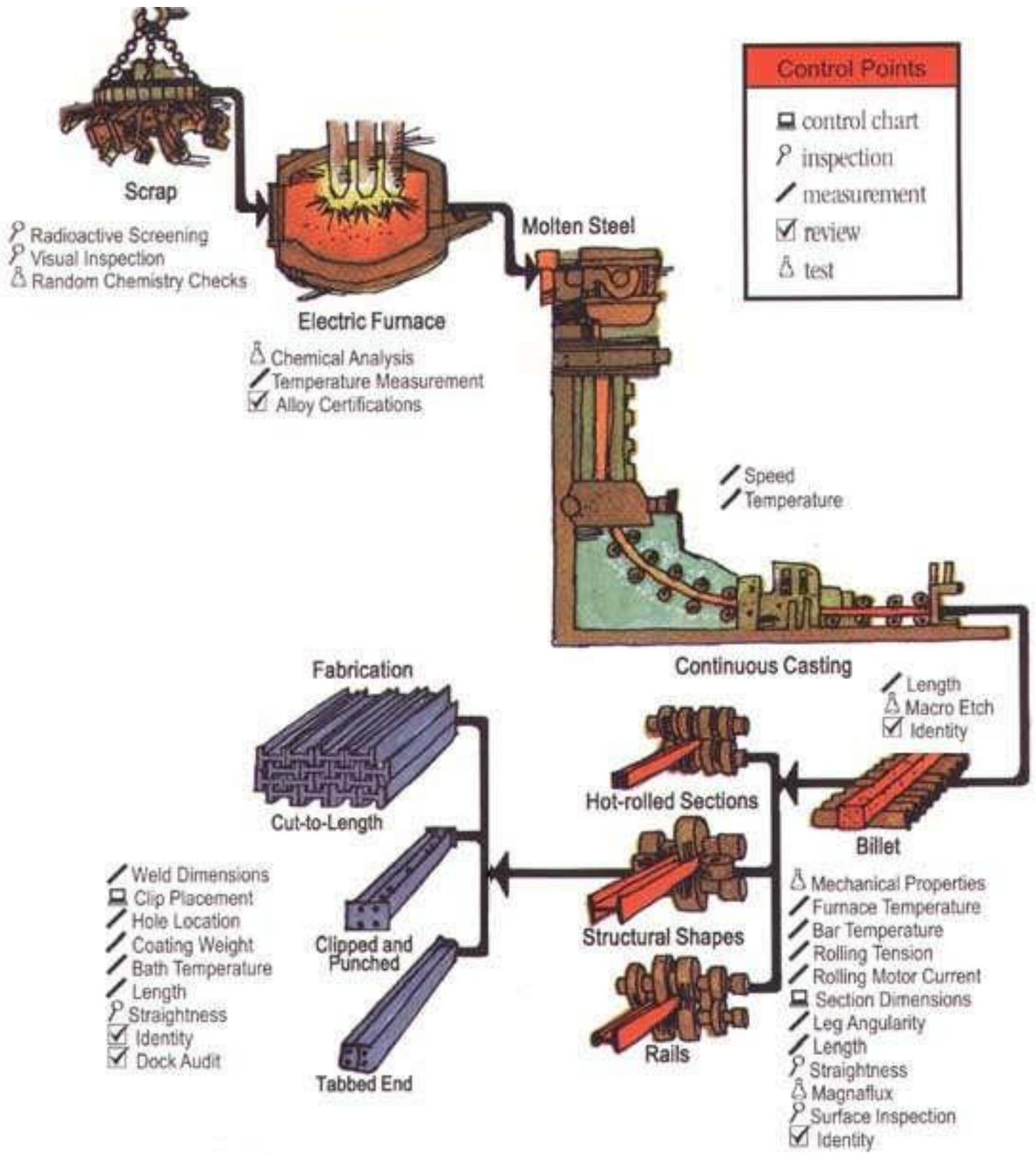

Figure 14: Company X's manufacturing processes 


\section{e) Energy bills and graphs:}

Companies are required to send their recent 12 months utilities to the IAC. Energy consumption and the corresponding energy costs for one year at company X studied from the recent 12 months electricity and natural gas billing details. For example, one electricity bill is shown in Figure 15.

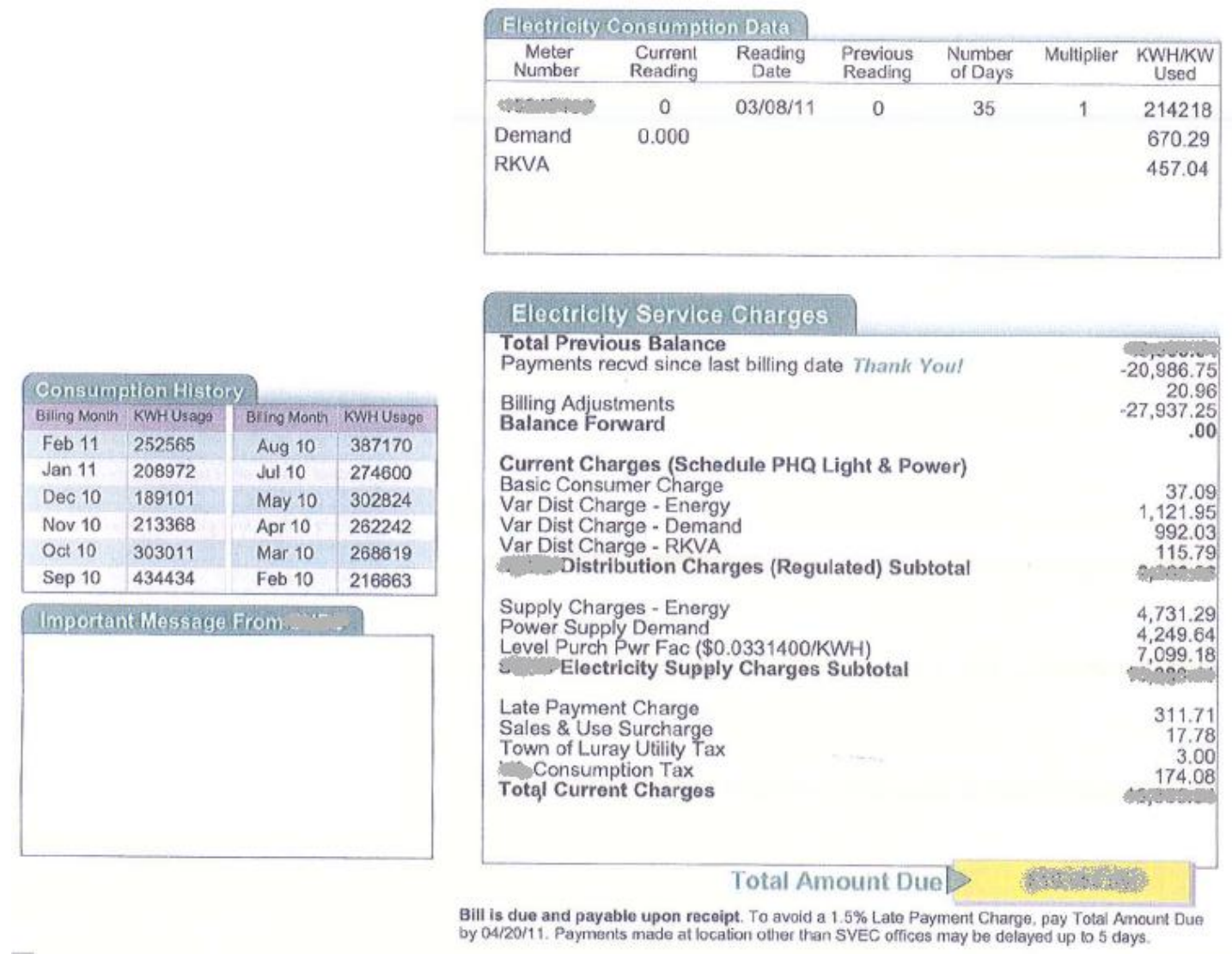

Figure 15: Sample electricity bill ${ }^{3}$

Utility analysis identifies significant information such as trends in consumptions, seasonality changes, range of consumptions, etc. The final results should be uploaded

\footnotetext{
3 The bill used as an example may be or not be for the respective company.
} 
in figure and table formats in the company profile. According to the bills, energy consumptions in 2009 at company $\mathrm{X}$ are shown in Table 5.

Table 5: Energy consumptions in 2009 at company X

\begin{tabular}{|c|c|}
\hline \multicolumn{2}{|c|}{ Electricity } \\
\hline MMBtu & Dollars \\
\hline 588,845 & $\$ 7,939,853$ \\
\hline
\end{tabular}

\begin{tabular}{|c|c|}
\hline \multicolumn{2}{|c|}{ Natural Gas } \\
\hline MMBtu & Dollars \\
\hline 658,494 & $\$ 2,909,226$ \\
\hline
\end{tabular}

Figures 16-18 resulted from utility analysis present further details about the energy consumptions at the company X. Table 5 and Figures 16-18 are then uploaded into the company X's profile, at the respective section for utility analysis. The complete results of utility analysis are presented in several tables attached in the company profile.

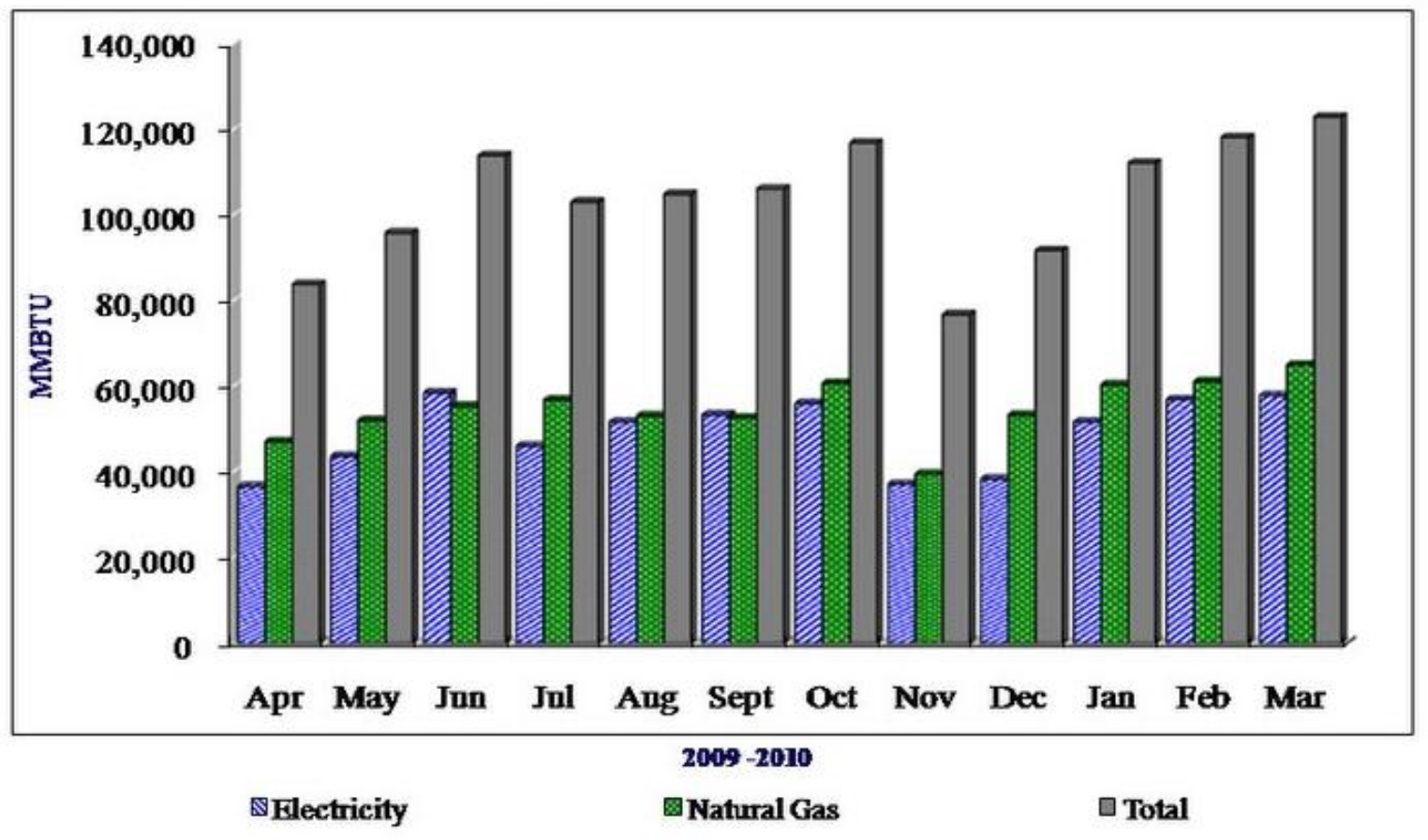

Figure 16: Utility Analysis, energy usage 


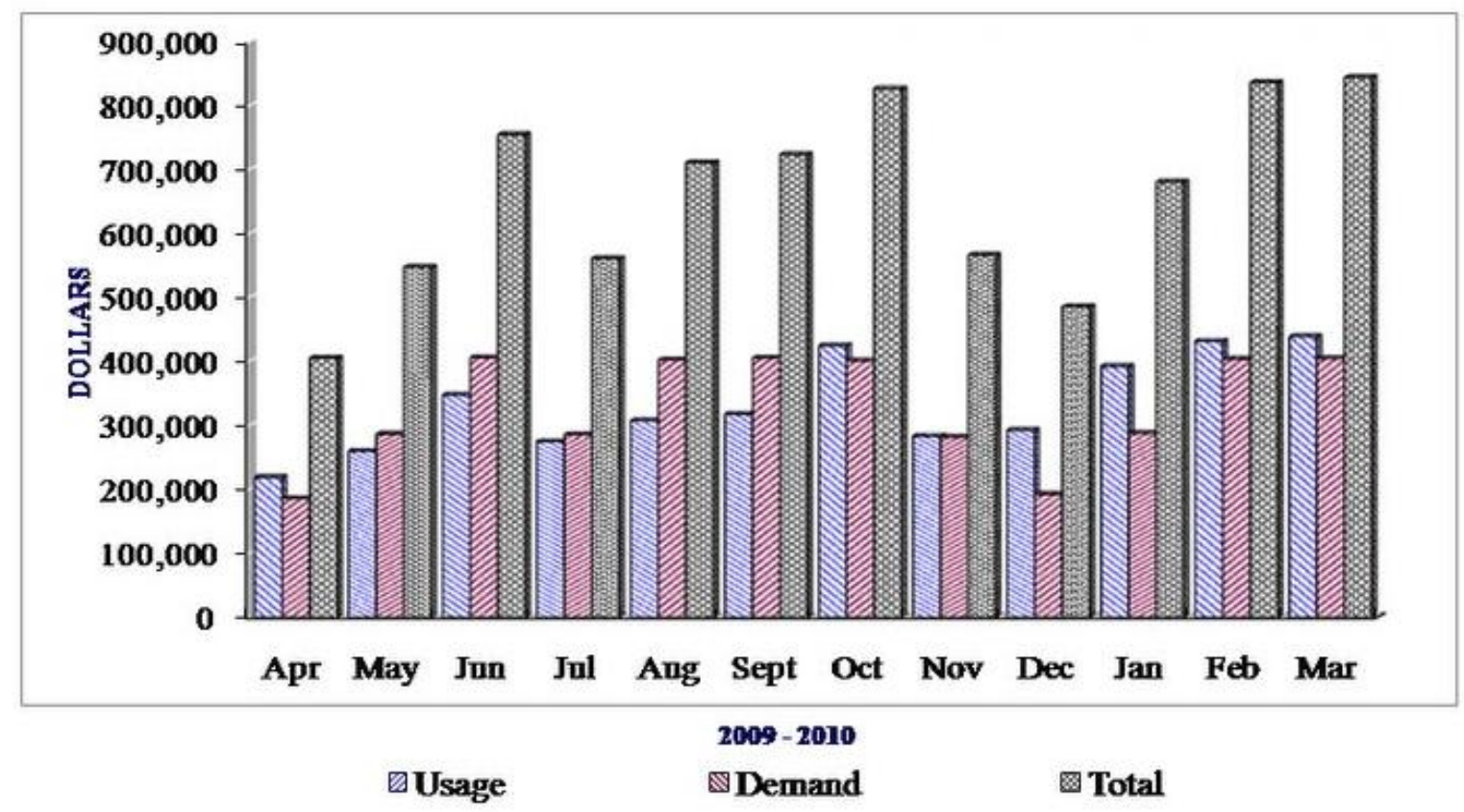

Figure 17: Utility Analysis, electricity cost

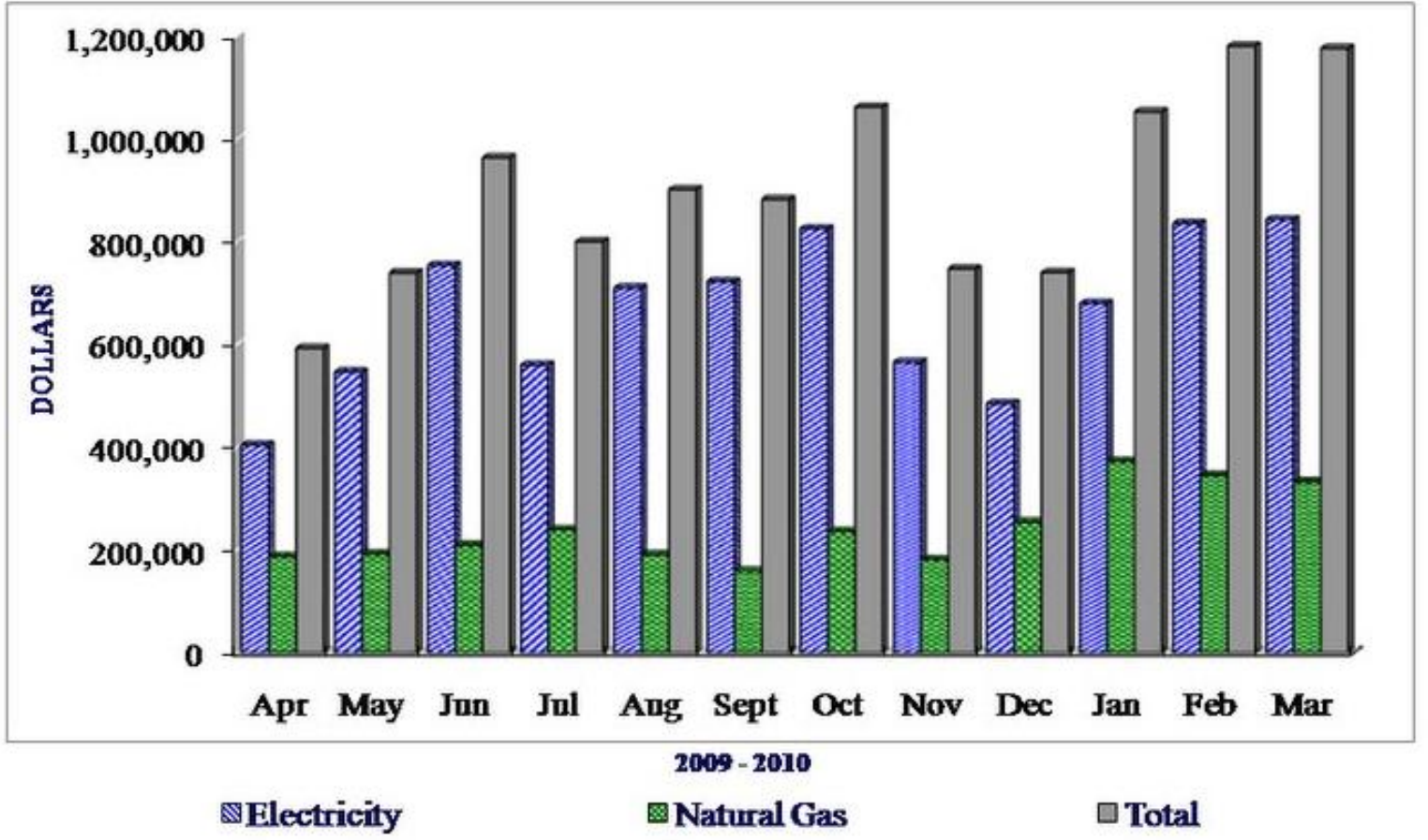

Figure 18: Utility Analysis, energy cost 


\section{f) QuickPEP:}

Based on the data obtained from the utility bills and the questionnaire, the amount of energy consumption in different energy systems found in the QiuckPEP software is shown in Figure 19.

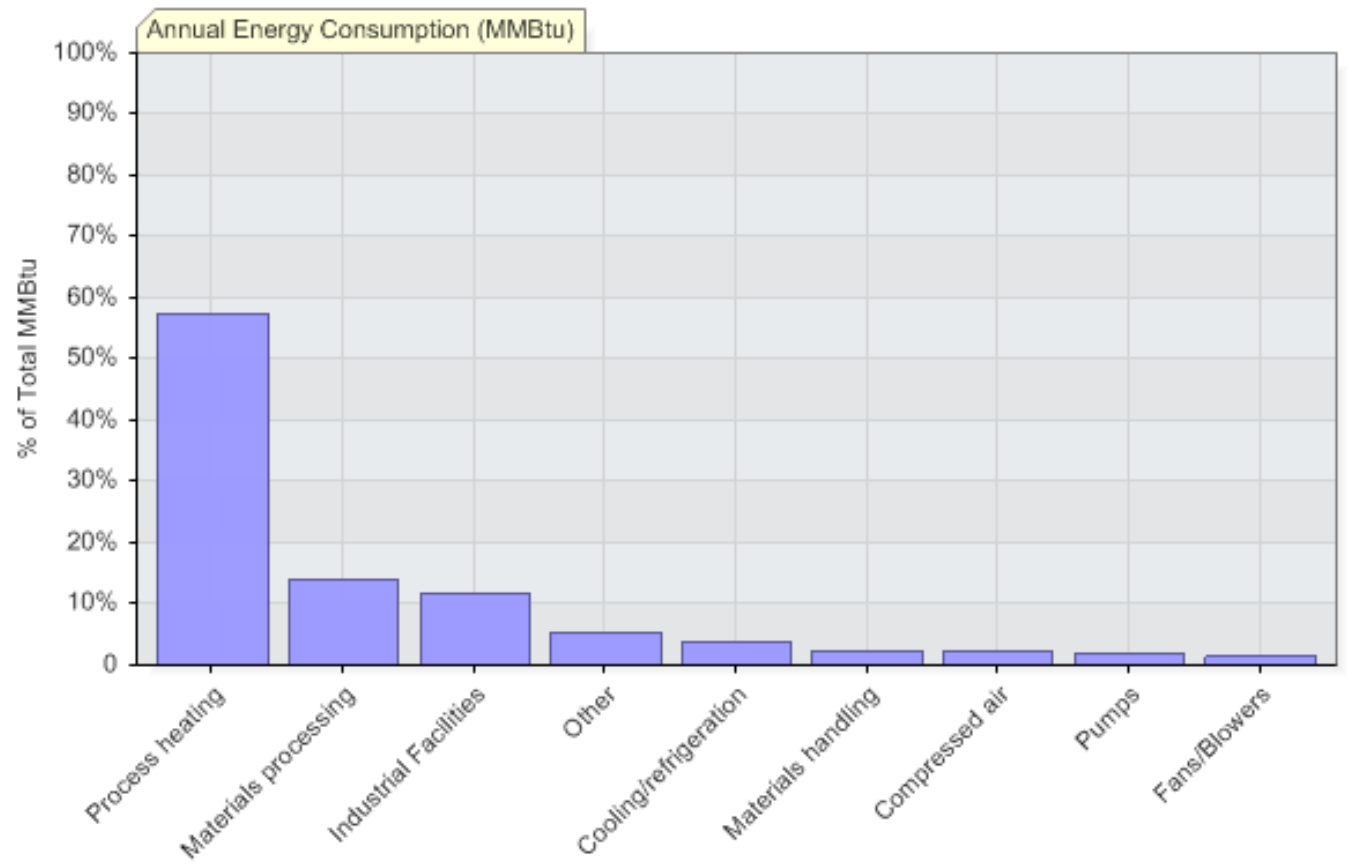

Figure 19: Energy consumption profile for the company X's plant

The following measures are recommended by QuickPEP to improve the energy efficiency of the company X's plant. It may be also noted that these measures are based on industry averages and does not represent the fact based analysis. The QuickPEP results are uploaded in the respective section of the company X's profile.

\section{i. General Energy Next Steps}

- Establish required payback period (or rate of return or discount rate) with due considerations for available incentives for energy efficiency improvement projects. 
- Promote use of life cycle cost analysis to evaluate economics of energy efficient equipment or system for all purchases that consume significant amount of energy.

- Develop methods for communication and feedback on energy use with a mechanism (or method) of receiving suggestions on possible opportunities for energy reduction from the employees. If possible include an incentives/reward plan.

- Form an energy management team and establish a position of facility energy manager who would be responsible for implementation of energy management plans and will provide periodic feedback on progress to the management.

- Develop a formal written energy management plan that is approved by the CEO or Board and includes specific annualized energy reduction goals in terms of percentage energy reduction.

ii. Process heating - $($ Potential Annual Savings $=\$ 730,200) *$

- Operate the furnace at or close to design load by proper furnace scheduling and loading- avoid delays, waits, cooling between operations etc. as much as possible.

- Keep heat transfer surfaces clean by eliminating build up of soot, scale or other material.

- Reduce or eliminate openings in the furnace to reduce radiation heat losses. Repair cracks and damaged insulation in furnace walls, doors etc. Keep the door opening to minimum during operations.

- Measure oxygen (O2) and Carbon Monoxide CO or combustible in flue gases and take actions to reduce $\mathrm{O} 2$ in flue gases while maintaining near zero value for $\mathrm{CO}$ or combustibles. In certain cases safety requirements may require to have high values of $\mathrm{O} 2$ in flue gases. Consult your equipment supplier before making any changes.

- Conduct a detail energy assessment for your heating equipment using tools such as Process Heating Survey and Assessment Tool (PHAST) to identify energy saving opportunities.

iii. Industrial Facilities: (Lighting, HVAC, and Facility Support) - (Potential Annual Savings $=\$ 175,800) *$

- Shut-off steam / chilled water flows to air handlers that are not needed or are out of service

- Implement night setback and weekend/vacation temperature / ventilation controls 
- Install occupancy sensors

- Evaluate lighting upgrades that includes implementing group relamp \& maintenance programs

- Perform a detailed Lighting \& HVAC System Assessment at your site to identify and quantify energy saving opportunities

iv. Process cooling and refrigeration - (Potential Annual Savings $=\$ 95,300) *$

- Float head pressure to minimum possible

- Evaluate use of variable speed drives on chilled water pumps, cooling tower fans, etc.

- Ensure proper refrigerant charge and eliminate non-condensable from the system

- Implement chilled water temperature and condenser water temperature reset

- Use the DOE CWSAT software tool \& other resources to identify and quantify energy saving opportunities

- Perform a detailed Refrigeration / Process Cooling System Assessment at your site

v. Pumps - $($ Potential Annual Savings $=\$ 79,400) *$

- Turn pumps ON and OFF to match needs

- Explore the potential for using a fixed speed pump to supply base load and a smaller, properly sized fixed speed pump for trim

- Evaluate the use of adjustable speed drives on pumps that have variable flow and are being throttled

- Use the DOE PSAT software tool \& other resources to identify and quantify energy saving opportunities

- Perform a detailed Pumping System Assessment at your site

vi. Compressed air $-($ Potential Annual Savings $=\$ 59,500) *$

- Reduce compressor operating pressure with or without controls

- Eliminate inappropriate uses of compressed air

- Implement air leak management program

- Use the DOE AirMaster+ software tool \& other resources to identify and 
quantify energy saving opportunities

- Perform a detailed Compressed Air System Assessment at your site

vii. Fans and Blowers - $($ Potential Annual Savings $=\$ 19,100) *$

- Minimize leakage and perform tightness tests, if needed

- Improve O\&M practices such as belt tightening, cleaning fans and changing filters regularly

- Replace dampers, variable inlet vanes with electronic variable speed drives for meeting variable loads

- Evaluate and reduce "system effect" through better inlet and outlet designs and duct sizing

- Use the DOE FSAT software tool \& other resources to identify and quantify energy saving opportunities

- Perform a detailed Fan and Blower System Assessment at your site

* - High Energy/Cost Savings Opportunity

It may be noted that the final results of QuickPEP should be obtained with more complete information after the assessment and then the company profile must be updated.

\section{g) IAC Database (Benchmark):}

At the end of company profile page, the IAC database is linked in which assessments can be inquired based on main products. Since company X's product is steel, the link goes to the IAC database showing all reports with steel products. The search results can be then sorted based on date, state, IAC center, etc. Figure 20 presents the screenshot of the assessments with steel products in the IAC database.

EEKC users can use the database and benchmark the results of company $\mathrm{X}$ with other available reports, especially with companies with similar operating conditions. This is 
very helpful in pre-assessment phase that provides some general ideas about steel companies before the assessment.

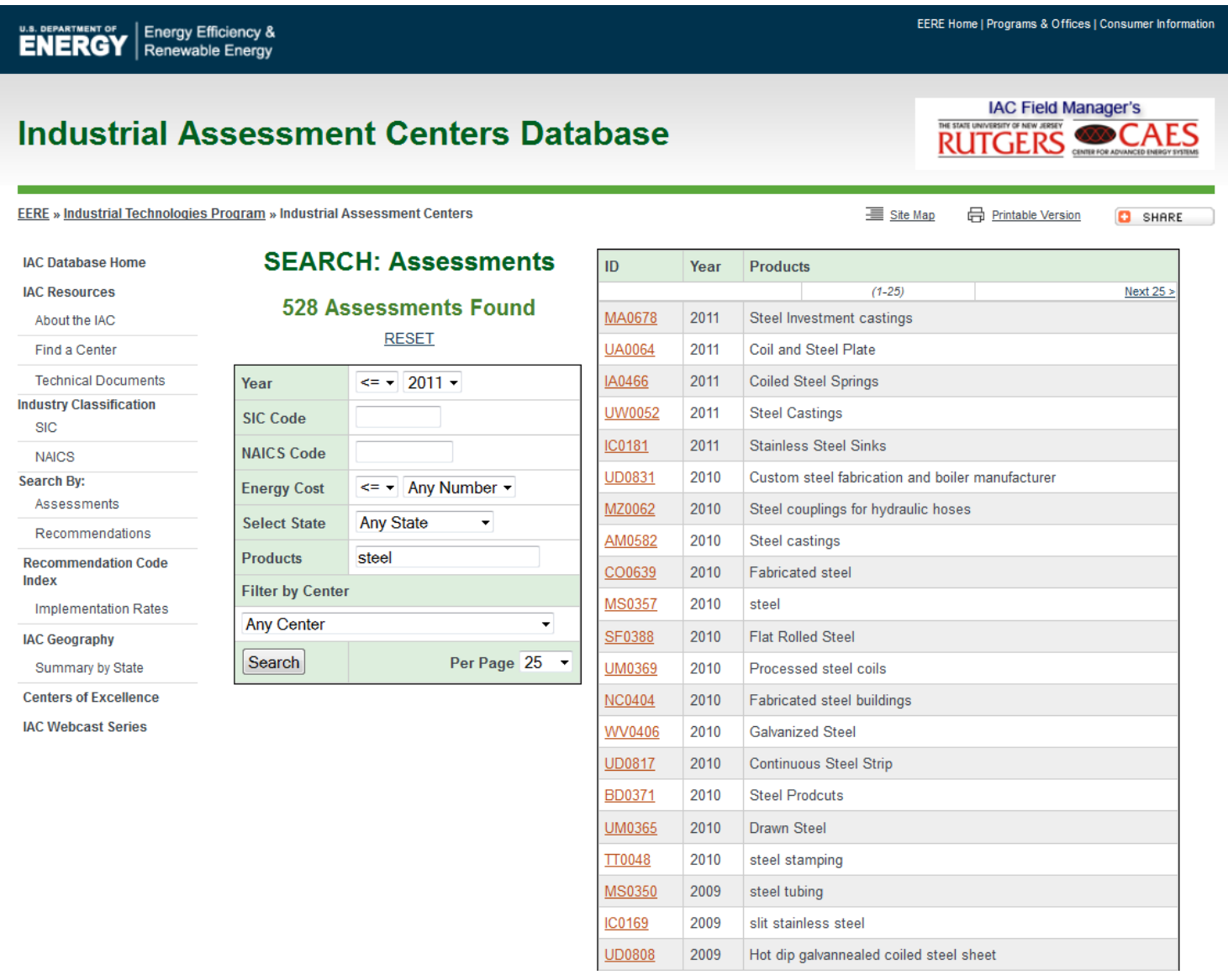

Figure 20: IAC Database, assessments for companies with steel product

\subsubsection{Assessment}

Data and information collection is the main activity during the assessments. Data gathered using data loggers must be analyzed and the results should be then saved for developing ARs and uploading in the company profile. Data logged of a compressor at company $\mathrm{X}$ is illustrated in Figure 21. 


\begin{tabular}{|r|r|r|}
\hline 1 & $3 / 8 / 201111: 53$ & 113.231 Logged \\
\hline 2 & $3 / 8 / 201111: 53$ & 113.231 \\
\hline 3 & $3 / 8 / 201111: 53$ & 113.044 \\
\hline 4 & $3 / 8 / 201111: 53$ & 113.231 \\
\hline 5 & $3 / 8 / 201111: 53$ & 113.044 \\
\hline 6 & $3 / 8 / 201111: 53$ & 113.044 \\
\hline 7 & $3 / 8 / 201111: 53$ & 112.95 \\
\hline 8 & $3 / 8 / 201111: 53$ & 112.95 \\
\hline 9 & $3 / 8 / 201111: 53$ & 112.95 \\
\hline 10 & $3 / 8 / 201111: 53$ & 112.95 \\
\hline 11 & $3 / 8 / 201111: 53$ & 113.231 \\
\hline 12 & $3 / 8 / 201111: 53$ & 112.95 \\
\hline 13 & $3 / 8 / 201111: 53$ & 113.138 \\
\hline 14 & $3 / 8 / 201111: 53$ & 113.231 \\
\hline 15 & $3 / 8 / 201111: 53$ & 113.231 \\
\hline 16 & $3 / 8 / 201111: 53$ & 113.419 \\
\hline 17 & $3 / 8 / 201111: 53$ & 113.606 \\
\hline 18 & $3 / 8 / 201111: 53$ & 113.606 \\
\hline 19 & $3 / 8 / 201111: 53$ & 113.681 \\
\hline 20 & $3 / 8 / 20111153$ & 113.681 \\
\hline 21 & $3 / 201$ & 3 \\
\hline & & \\
\hline
\end{tabular}

Figure 21: Data logged of a compressor pressure at company $\mathrm{X}$

This data profile of the compressor pressure should be analyzed and then illustrated in figures. For instance, data gathered of a compressor in company $\mathrm{X}$ is illustrated in Figures 22-23.

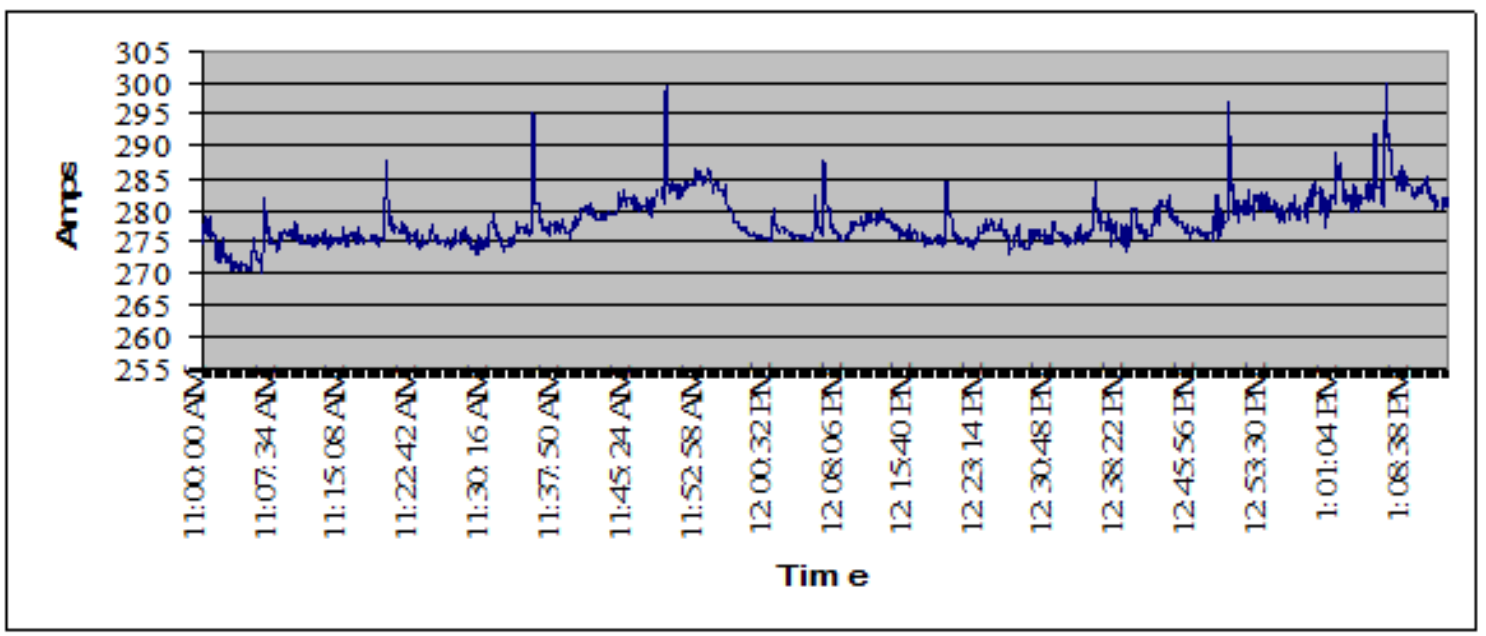

Figure 22: Current Profile of a compressor at company $\mathrm{X}$ 


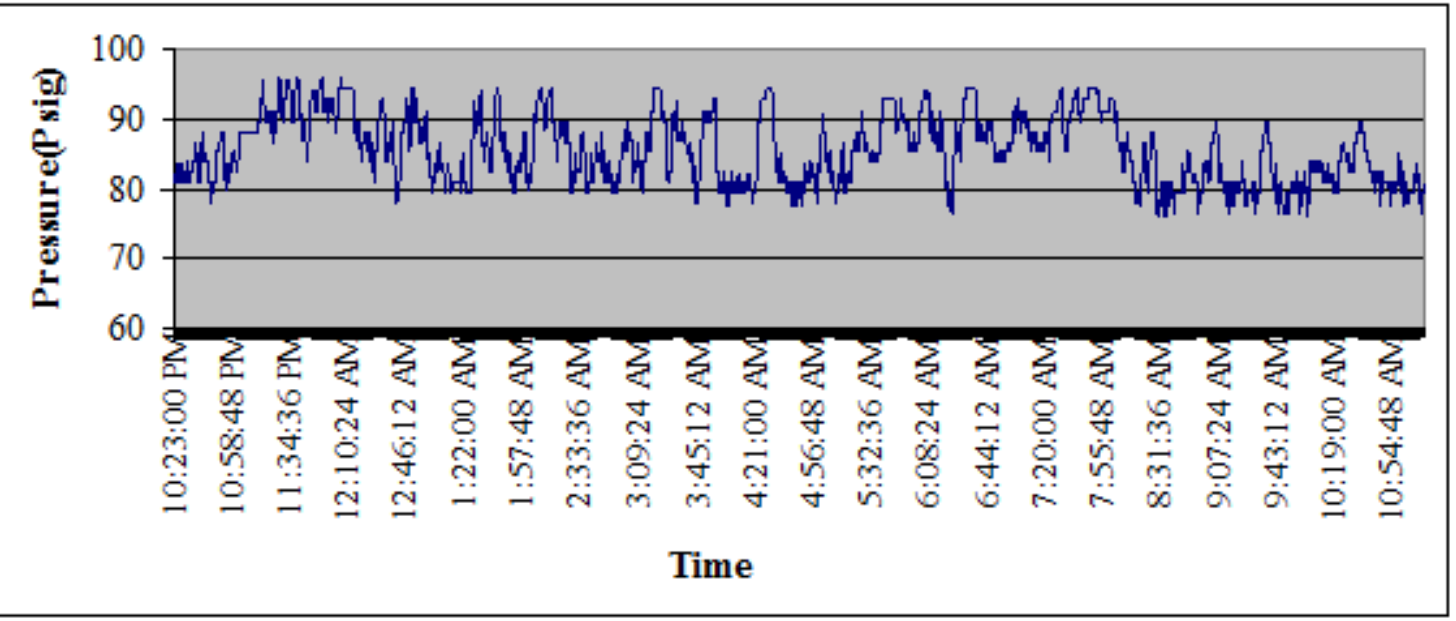

Figure 23: Pressure profile of a compressor at company $\mathrm{X}$

According to information gathered during the assessment, best practices applied at the company $\mathrm{X}$ are found as follows:

\section{Best Practices:}

1. Use synthetic lubricant in compressors

2. Check vibration levels for preventive maintenance of the motors

3. Use Automatic Inspection System

4. Scrap used in furnace combustion

5. Curtains in charging ends of number two furnace avoids heat loss

6. Furnaces have two controls, software and thermo couples

7. High profile laser gauge for quality checks

Then, the best practices are uploaded in the company profile.

\subsubsection{Post-assessment}

Post assessment results and information and knowledge extracted from each AR are uploaded in the company profile. The assessment results for company $\mathrm{X}$ is presented in Table 6. 
Table 6: Energy assessment results (summarized)

\begin{tabular}{|c|l|l|}
\hline \multirow{2}{*}{ Current usages } & Total energy used & $1,247,339 \mathrm{MMBtu} / \mathrm{yr}$ \\
\cline { 2 - 3 } & Total energy costs & $\$ 10,849,079$ \\
\hline \multirow{2}{*}{ Savings } & Savings by implementing ARs & $81,909 \mathrm{MMBtu} / \mathrm{yr}$ \\
\cline { 2 - 3 } & Total energy cost savings & $\$ 739,627$ \\
\cline { 2 - 3 } & Total energy cost savings & $7 \%$ of the annual energy costs \\
\hline \multirow{3}{*}{ Implementation } & Total estimated implementation cost & $\$ 896,285$ \\
\cline { 2 - 3 } & Average simple payback & 15 months \\
\hline
\end{tabular}

\section{Layout and Process Flow Diagrams:}

Layout and process flow diagrams are usually prepared in post-assessment reports. The diagrams for company X are presented in Figures 24-25.

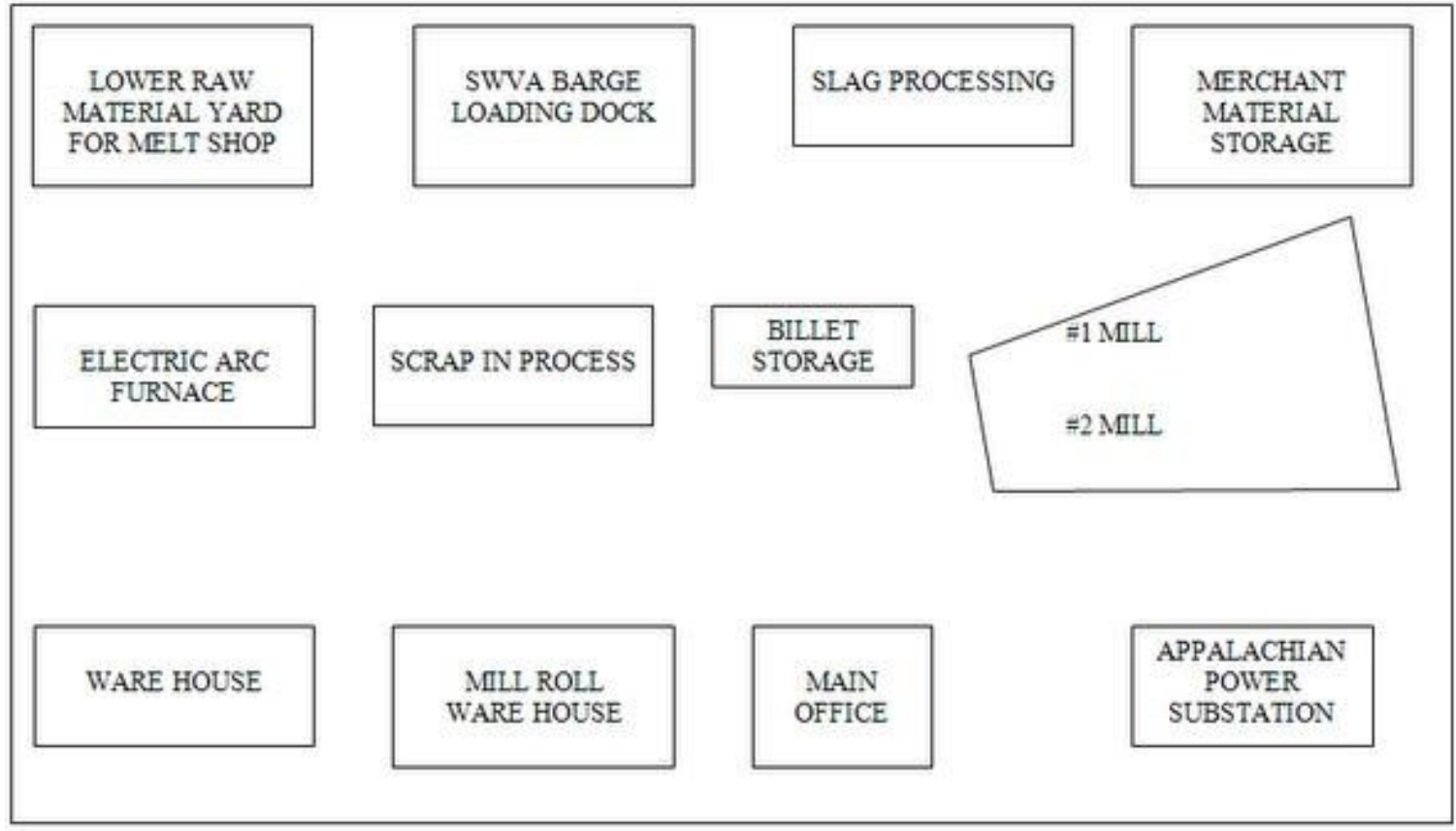

Figure 24: Company X's layout 


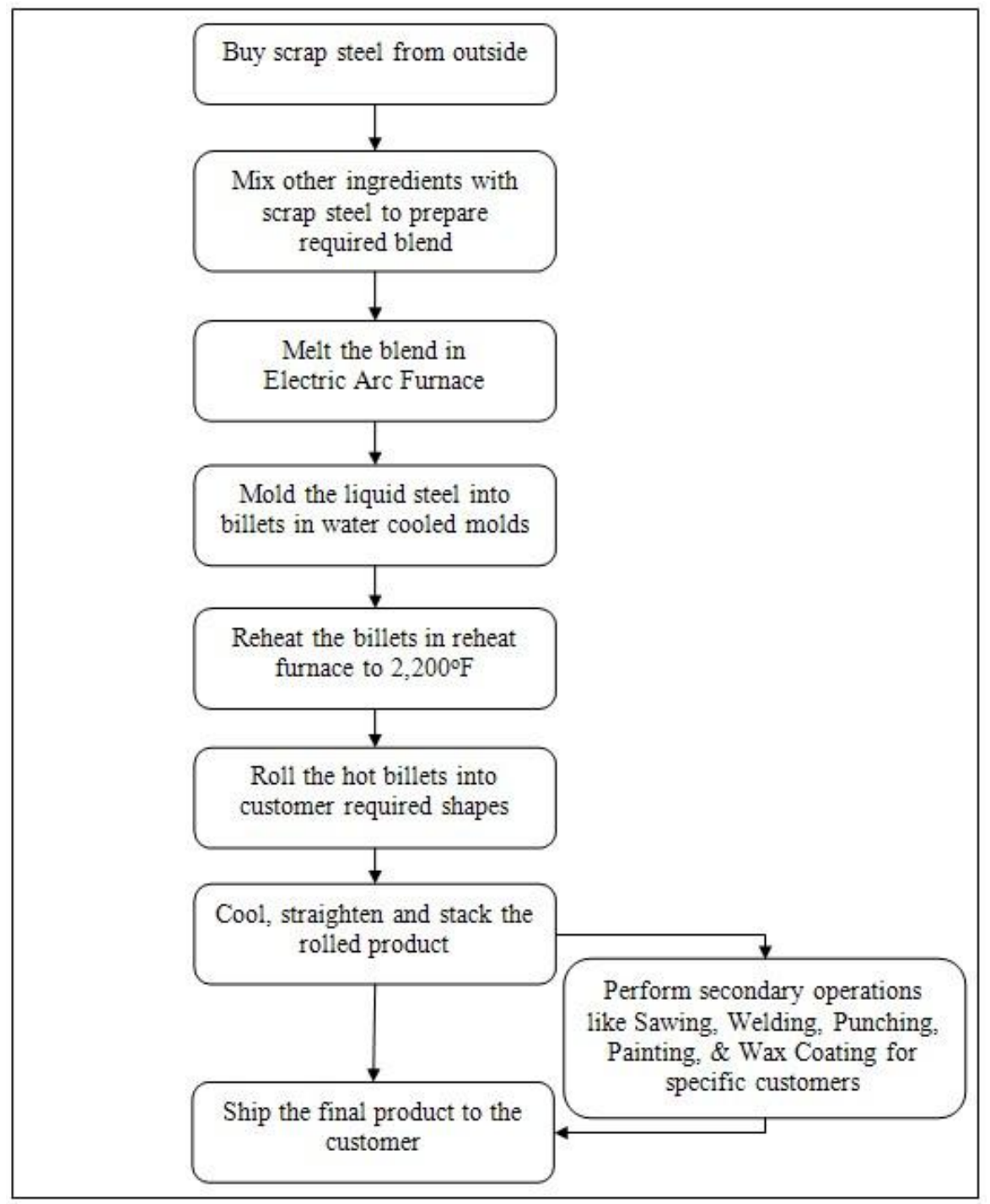

Figure 25: Company X's process flow

The layout and process flow diagrams are uploaded in the company profile.

\section{Assessment Recommendations}

The most important part of the company energy profile is the AR section. As it was illustrated in Figure 13, each AR is connected to the Wiki page, file archive, comments, rebate and incentives, and vendor information. Each AR should be summarized with 
practical information and then presented in the company profile. For instance, the information extracted out of a nine-page AR is as follows:

Recommendation: Monitor demand pattern and schedule all the equipment accordingly to reduce the demand cost.

Description: Peaks in demand are caused by a number of factors. One of these factors is the operation of equipment that consumes much power like motors, compressors, etc. Demand cost can be reduced via systematic scheduling of the equipment based on the monitored demand pattern. The current profile of the energy and demand is shown in Figure 26.

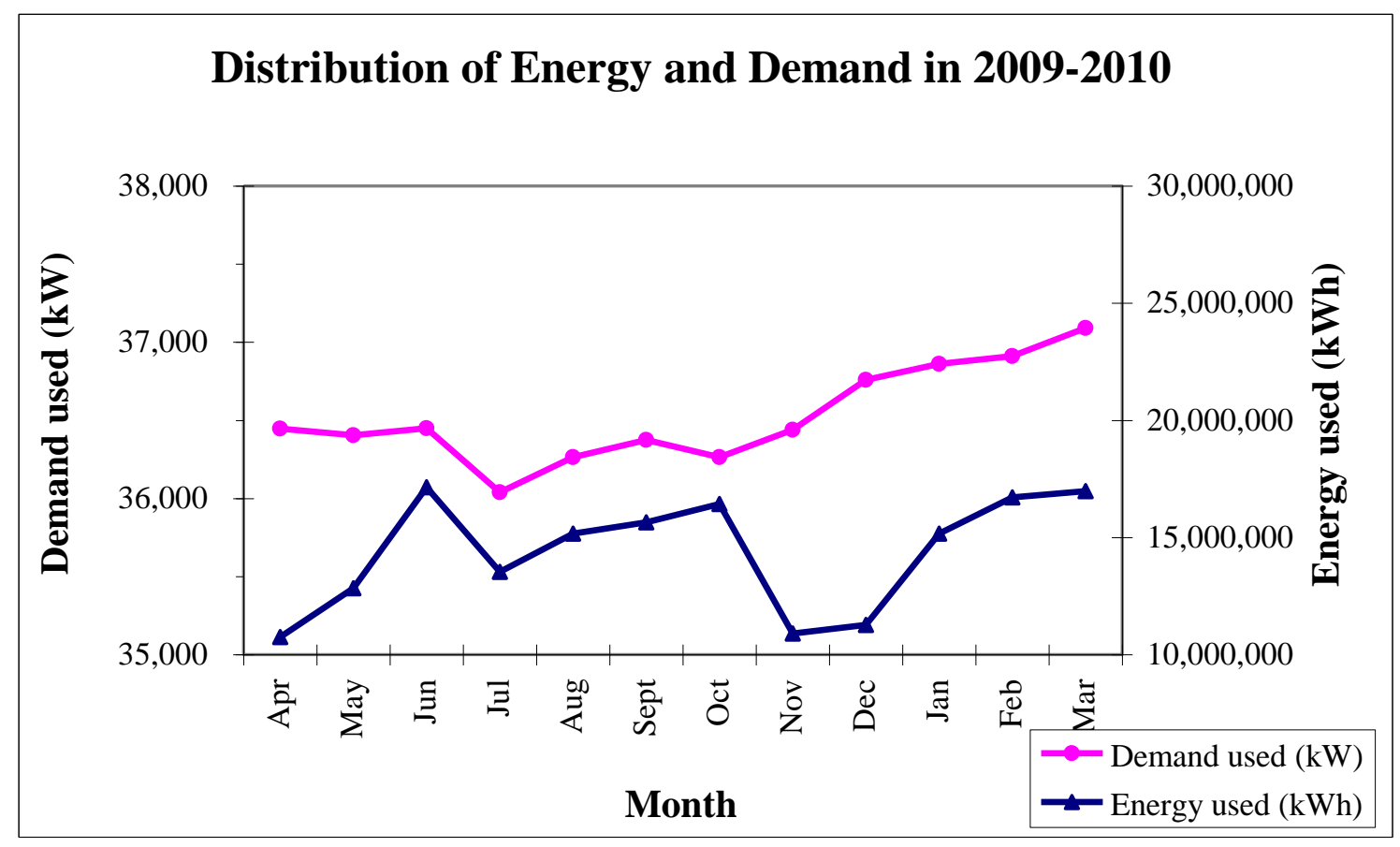

Figure 26: Demand and energy usage profile in 2009-2010 
To estimate the reduction in the peak demand, the average hours of operation $(\mathrm{kWh}$ per $\mathrm{kW})$ is calculated based on the energy $(\mathrm{kWh})$ and demand $(\mathrm{kW})$ used during a month. The distribution of this metric is shown in Figure 27.

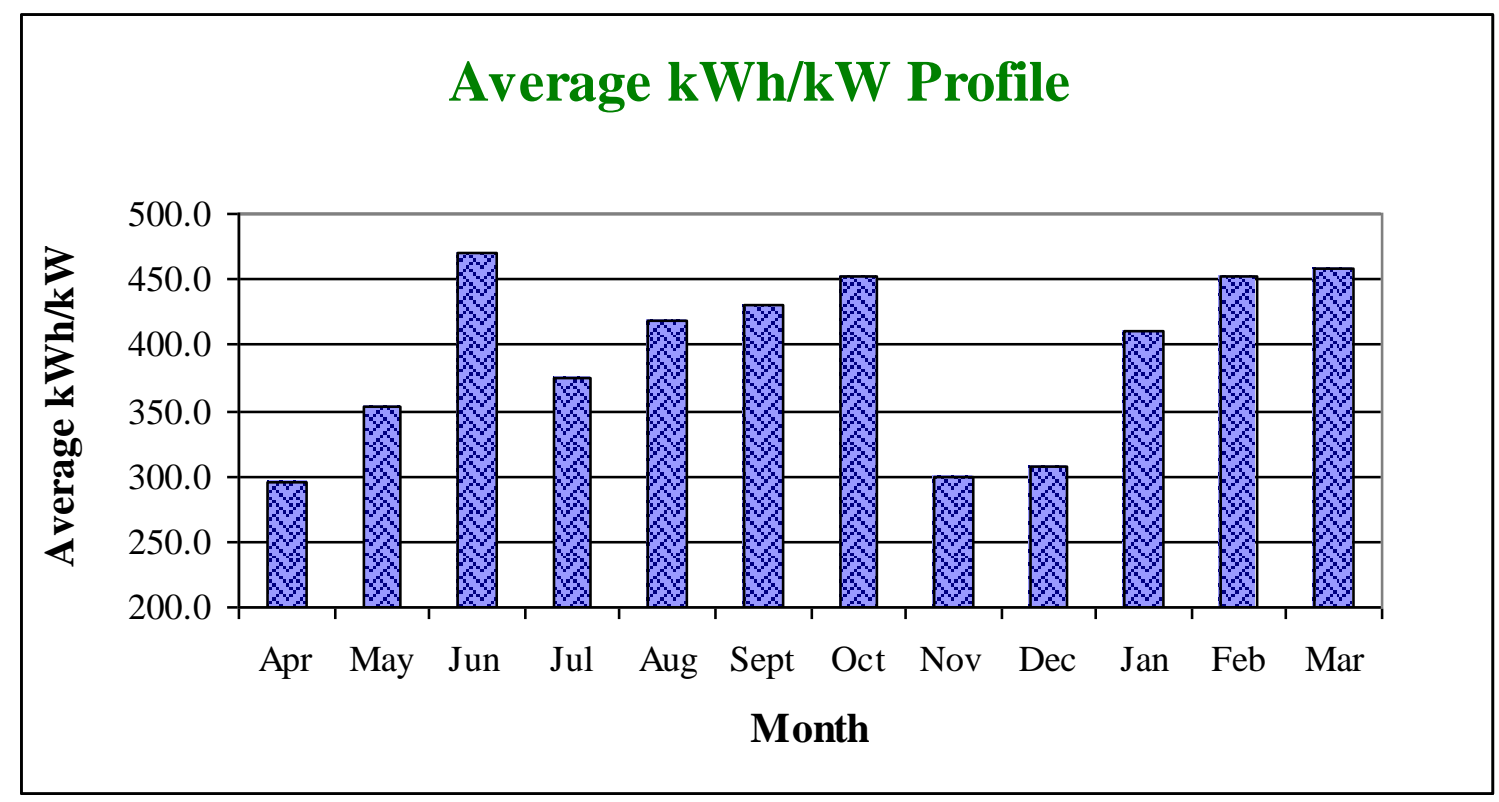

Figure 27: Average $\mathrm{kWh} / \mathrm{kW}$ distribution in 2009-2010

In figure 27, a value of 400 or more is practical and is achieved in June, August, September, October, January, February, and March. It is evident that a high value of this ratio results in lower peak demand and can reduce the demand charges during the month. Assuming that a value of 400 for this ratio can be achieved in each month, the expected demand and the demand cost savings can be calculated. Accordingly, the savings and implementation costs would be,
Cost Savings
$=\$ 275,195 / \mathrm{yr}$
Implementation Costs $=\$ 8,840$
Payback Period
$=1$ month 
The presented AR could be even more summarized. To provide more information about the AR, AR full text is linked right after the AR summery. The main and important factor is not only to summarize the AR, but also it is to develop the Wiki page of the AR that provides information and knowledge that are not presented in the report.

Hence, each AR is shortly presented along with links to the complete AR file, attachments such as Excel files, Wiki page (including general information, knowledge and useful tools for that AR), vendor information, and rebates and incentives. Those links for each AR are also accessible through the Knowledge Center page of the EEKC.

\subsubsection{Knowledge Center}

Knowledge Center shown in Figure 28 is accessible through the site navigator. The categories and subtitles shown in Figure 28 are created based on the report selected for the case study. For future assessments, more categories and subtitles can be added. DOE tip sheets are also available in the related page linked into the Knowledge Center page. The Knowledge Center section becomes more and more comprehensive over time. 


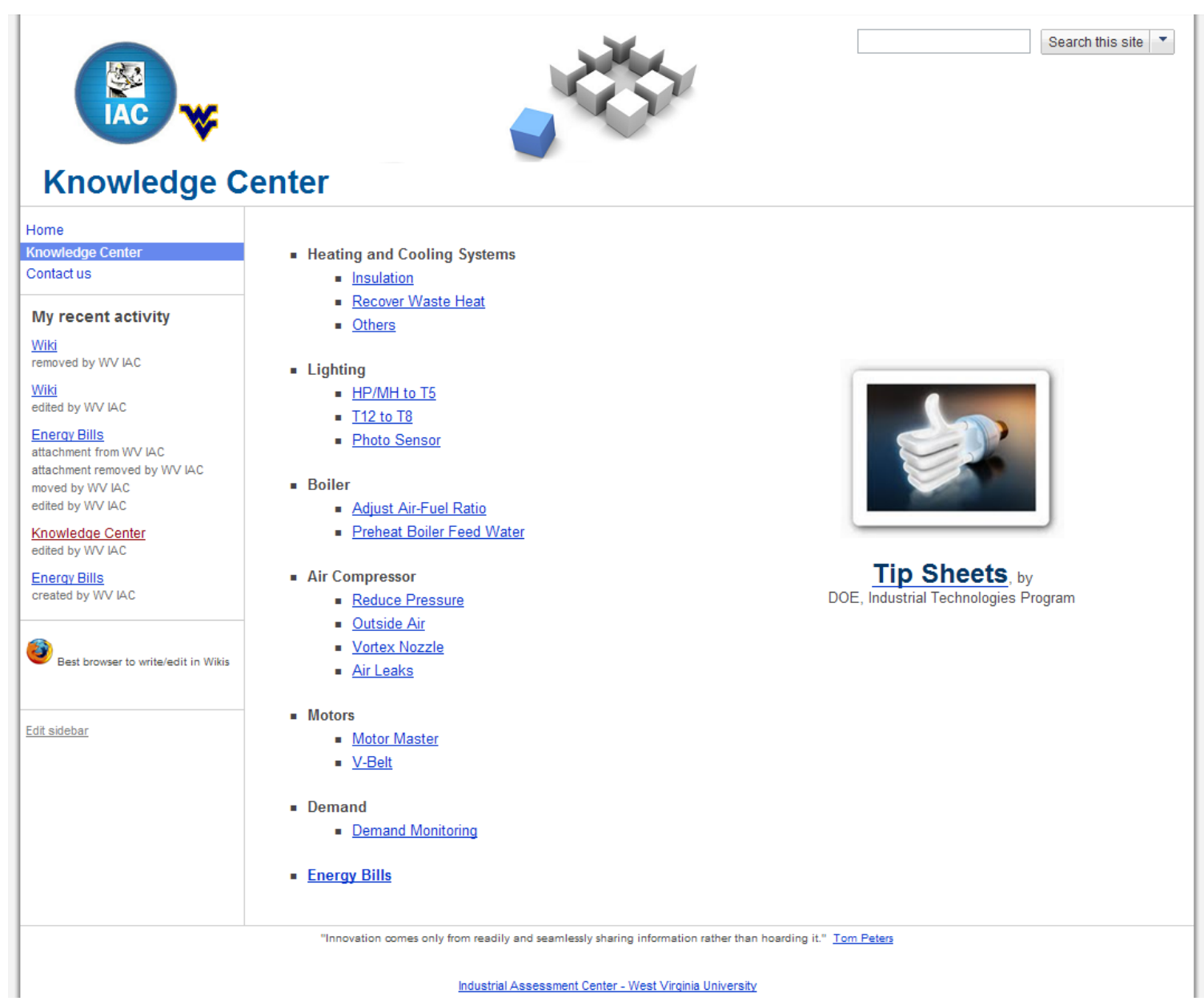

Figure 28: Different categories presented in knowledge center page

As it was mentioned earlier, each subtitle in the Knowledge Center page is a specific kind of AR (Figure 28) which links to a particular page including AR file, Wiki, vendor information, and rebates and incentives. For example, "Insulation" subtitle which is under "Heating and Cooling Systems" is illustrated in Figure 29. 


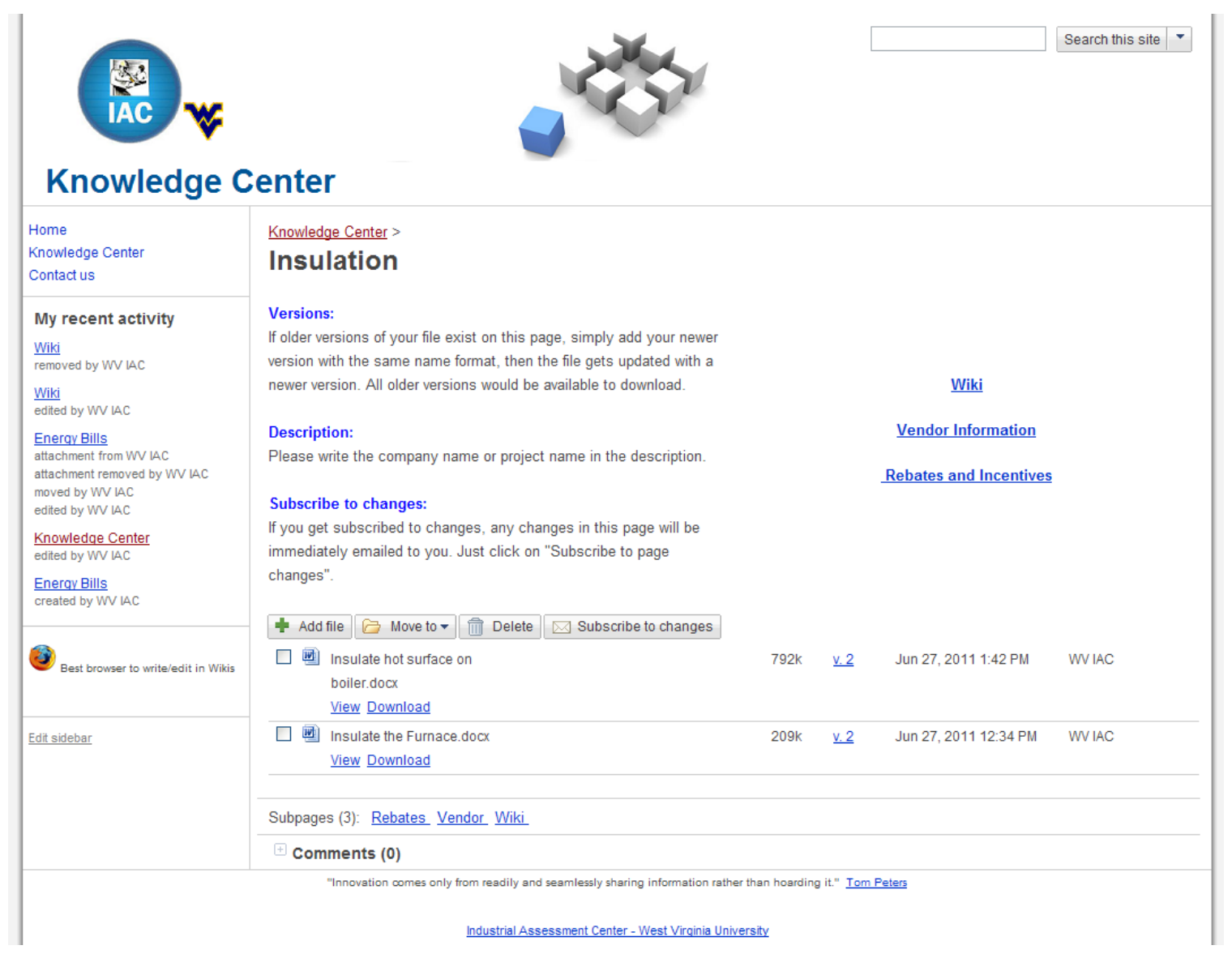

Figure 29: An example for AR bank (Insulation)

The Wiki link presented in Figure 29 enters to a sub-page designated to save and share knowledge and hands-on experience about insulation. All IAC members should update these Wiki pages. The IAC members can easily edit Wiki pages with text, figures, tables, spreadsheets, videos, web forms, etc. All they need to do is just typing in the pages, or uploading their files. Figure 30 presents a Wiki page for Recover Waste Heat AR. As it is shown in Figure 30, the page is filled with PDF links, text and figures. 


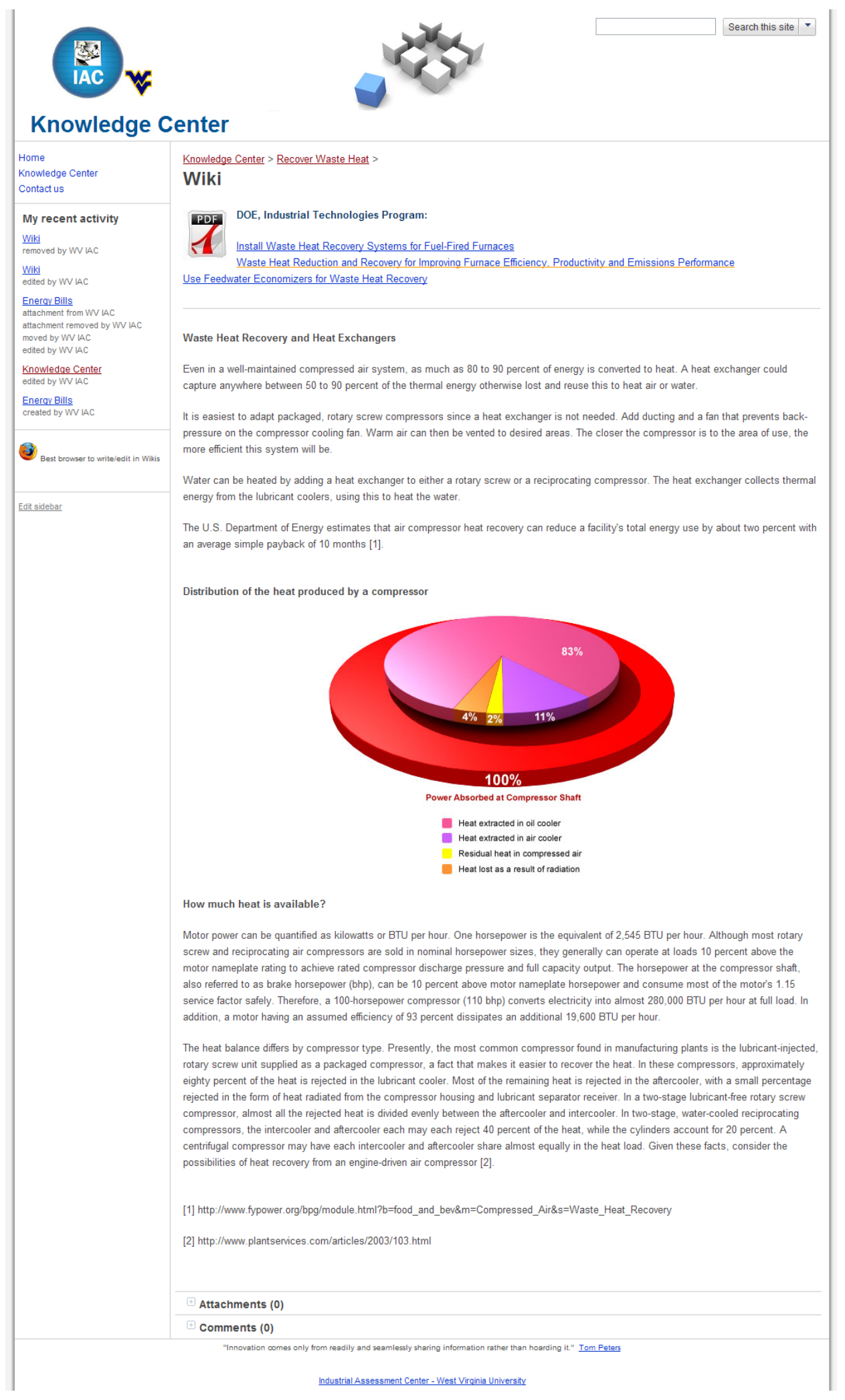

Figure 30: A Wiki page, (Recover waste heat) 
For some topics, it would be more effective if a reference i.e. a PDF file is shown directly in the page right after text without linking to outside the site. Figure 31 shows an example page, Outside Air Wiki page, in which a reference file in PDF format is directly shown in the page. Other kinds of reference files and even a webpage can be shown as same as the PDF in Figure 31. Any user who has permission to edit the site can add references directly in the pages. The steps to do so are provided in site Help.

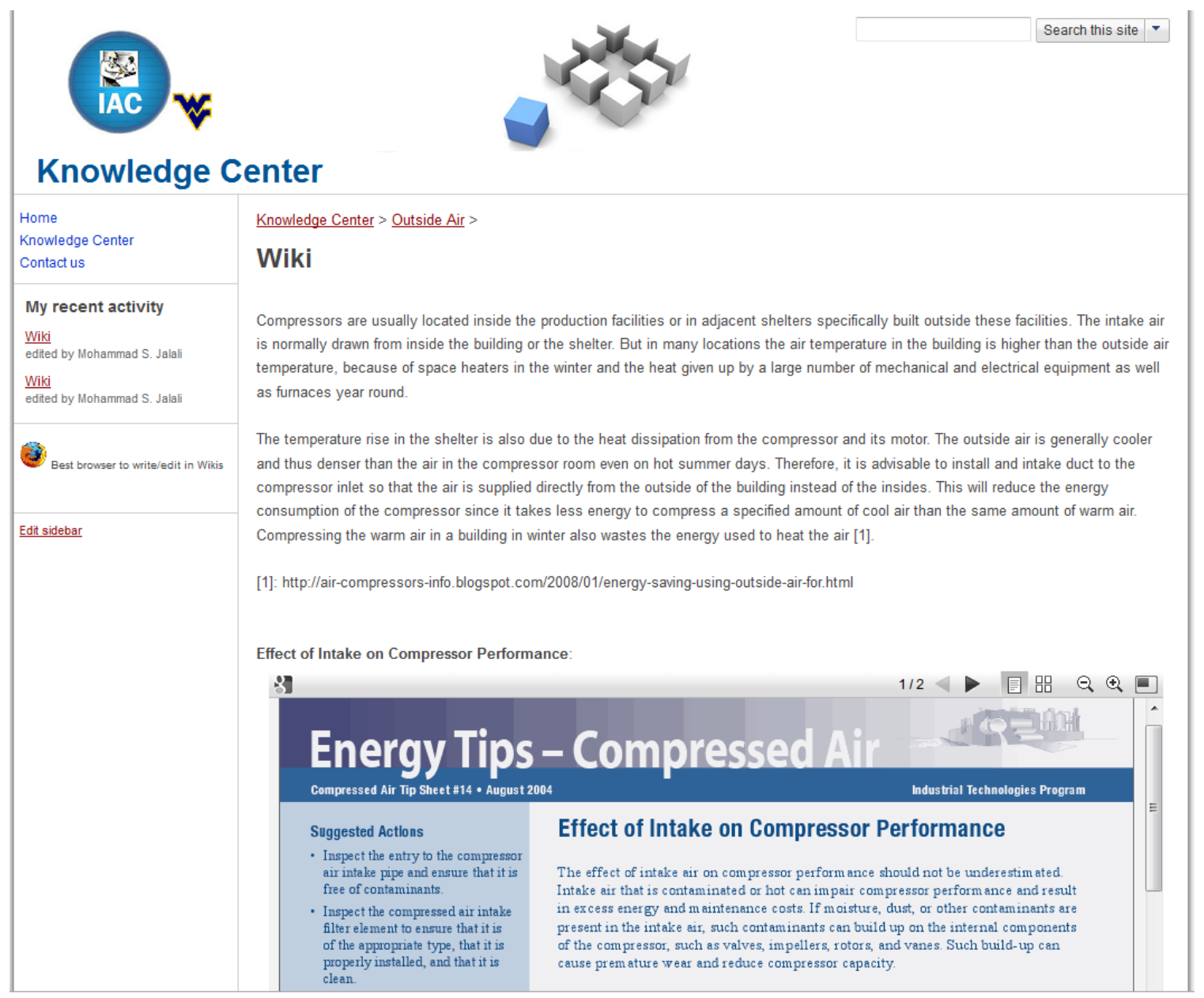

Figure 31: Showing a reference file directly in a Wiki page 
To ensure that all users can share their feedbacks on any content, a section to upload attachment and comments is designed on the down side of pages, as illustrated in Figure 32.

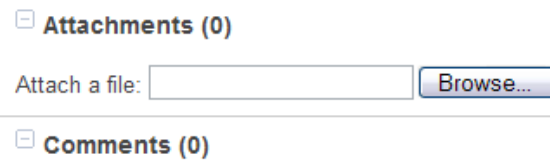

Figure 32: Attachment and comment illustration

\subsection{Security Protocols}

To login to the EEKC, users need to have a Google account which uses https protocol. The https provides encrypted communication and secure identification of a network web server. Https connections are often used for payment transactions on the web and for sensitive transactions in corporate information systems [21]. Https enables secure access to web content and web-based services. Threats like attackers gaining access to confidential account information or even redirecting transactions. The https protocol enables this protection and ensures confidentiality, integrity, and authenticity [22].

When using an https connection, the server responds to the initial connection by offering a list of encryption methods it supports. In response, the client selects a connection method, and the client and server exchange certificates to authenticate their 
identities. After this is done, both parties exchange the encrypted information after ensuring that both are using the same key, and the connection is closed [23].

Google uses a setting in Google accounts that lets users always use Secure Sockets Layer (SSL) when accessing their Gmail account and other Google services (rather than simply when the user logs in). In many web service providers, systems use SSL (indicated by the https://prefix in the address bar) to log users in, but then drop back into unencrypted http. That means that anyone accessing their data over an unencrypted Wi-Fi link runs the risk of having their packets sniffed over the air. If, as is common practice, passwords and usernames are sent via email, accounts could easily be compromised [24].

Google also added the ability to check whether another computer is logged into an account, and to check the latest activity on an account to be sure that only the user has access. This measure helps to prevent an attack made possible by the clear text flaw described above, in which an attacker could sniff a cookie passing over a local network and use it to stay logged into a user's account until that user actively logs off [24]. The login page to the private content of the EEKC is shown in Figure 33.

If anytime the IAC decides to set-up the EEKC on its own server and database, all the information and files could be saved and moved to any other server. The EEKC administrator has also ability to adjust the visibility of the public pages on search engines such as Google, Yahoo, Bing, etc. 


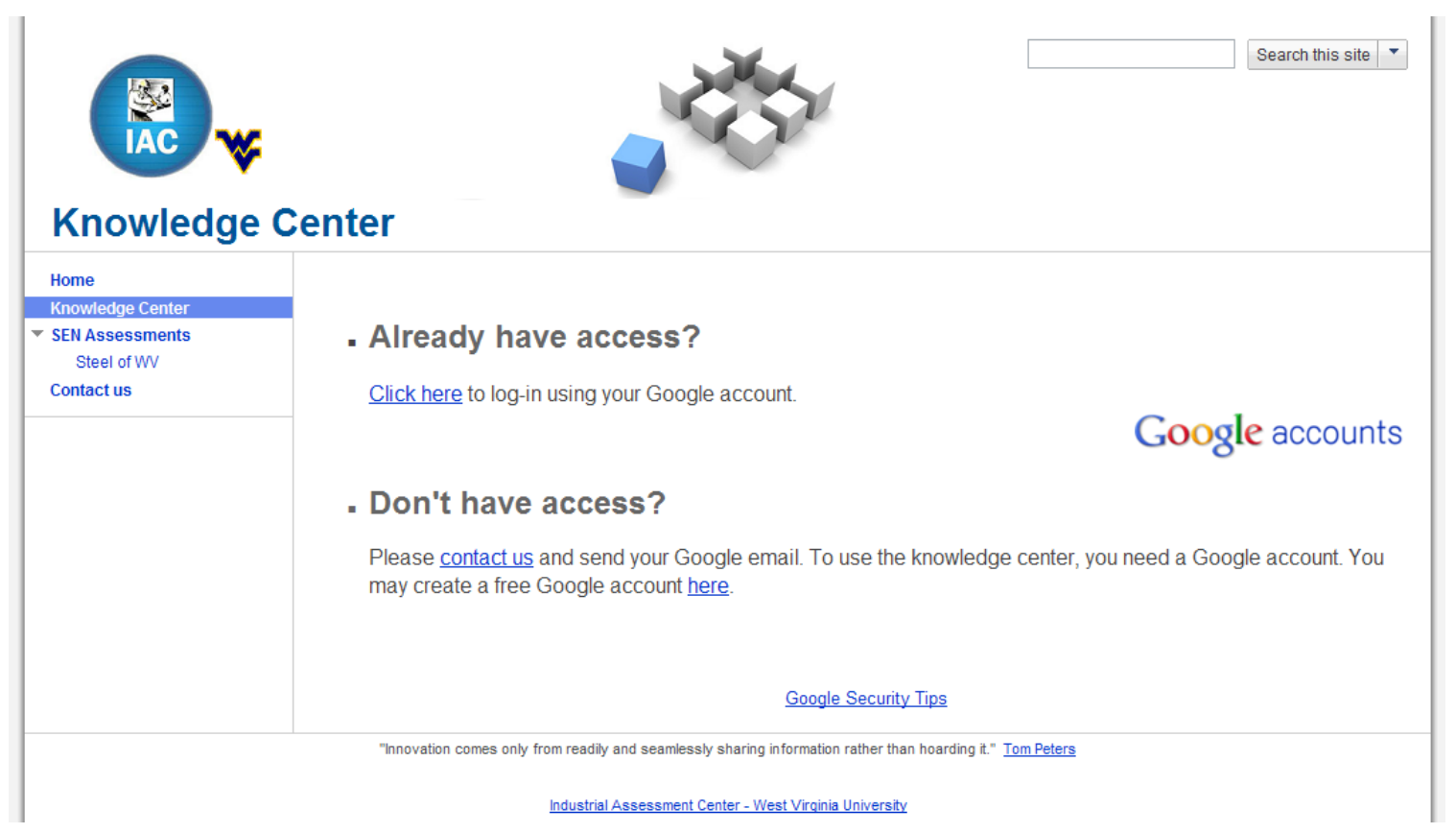

Figure 33: Login page interface

If the IAC directors or the EEKC administrator would like to give permission to some people to view or edit the private sections of the EEKC, they can easily invite those people by entering their email addresses into permission section on top of the page. While inviting new users to the system, their ability to view and edit can be defined. If one eligible to view or edit the EEKC pages does not have access, he/she needs to contact the administrator through the "Contact us" page and ask for access.

Who can view/edit the EEKC pages is absolutely controllable by changing the sharing settings. To increase the security levels, the EEKC is created with two websites, one public and one private. Obviously, everyone who knows the web address is able to view the public site. In the private site, it should be clearly specified which people can do certain actions on the site by setting their access level to "Can view," "Can edit," or "Is owner" [25]. These levels are explained as follows: 
- Users set to "Can view" can:

View pages

- $\quad$ Users set to "Can edit" can:

Create, edit, and delete pages

Move pages

Add attachments

Add comments

Subscribe to site and page changes

- Users set to "Is owner" can:

Do everything that users set to "Can edit" can do

Set other people as "Can view," "Can edit," and "Is owner"

Change site themes and layout

Change the site name

Delete the site

Permissions to clients are also controllable. It means that they can be given permission to view/edit only specific company profiles not the entire system. To do so, each company profile should be separately created and so permissions will be given only to desired company profiles. Hence, the administrator needs to set the access levels of users for each company profile. The company profiles must be absolutely separated from each other; however they are all connected to the knowledge center pages.

\subsection{Subscriptions}

The EEKC users can sign up to be notified by email whenever any changes happen. The steps to get notified are as follows: 
- Click the "More Actions" drop-down menu on the page for which you want notifications.

- Select "Subscribe to site changes" or "Subscribe to page changes" depending on the type of notifications you'd like to receive.

Once one is subscribed, an email will be sent each time the site or page is updated, someone makes a comment on the site or page, or someone uploads an attachment to the site or page. The email will highlight the additions and deletions to the site or page. Since users use their Google account to login to the system, the notification emails are sent to their Gmail [26]. If they prefer not receiving notification emails in their Gmail but in another email address, they can auto-forward their Gmail account to any other address. The steps to how to do this are available in the site help.

\subsection{Assessment Tool Case}

Assessment tool case which is available in the EEKC includes the checklist and essential questions created over a number of assessment projects for major energy systems. As it was mentioned earlier, for each particular AR, the required information may change if manufacturing parameters are different, that it might be difficult for nonexperienced members to catch all essential information. The assessment tool case helps the IAC members ensure that all assessment activities are accurately and effectively performed with minimal mistakes.

The checklists need to be developed and modified after each assessment. To start developing the assessment tool case, several checklists are developed for some of the major energy consuming equipment presented as follows [20]: 


\subsubsection{Air Compressor Checklists}

- Note technical characteristics of compressors and their drives from their labels.

- Check if the compressors are adequately sized.

- Does the control system of the compressor result to efficient operation? Check idling operation time.

- Is the pressure set to the lowest level possible to meet the operating requirements?

- Is the intake air being cooled?

- Check the possibility to decentralize the system if only single users need higher pressure.

- Check if compressed air is used only where it is absolutely necessary and unavoidable.

- Check if compressed air can be substituted by electricity at individual users.

- Identify the real pressure requirements of the individual users and check possibilities to reduce pressure in the distribution system or in parts of it by installing throttle valves in branches.

\subsubsection{Boilers Checklists}

- Check if a flow diagram is available in the boiler room and try to obtain a copy.

- If not available, draft a simple one.

- Look if a logbook is maintained and obtain copies for a typical week.

- Note the technical specifications of the boilers and burners from their labels.

- $\quad$ Note the operating pressure and temperature. 
- Check if metering and control instruments are installed.

- Check if there is a hole in the exhaust gas track for the combustion analyzer. If not ask operators to drill one.

- Check for water and steam leaks.

- Check if boiler, pipe and condensate tank are adequately insulated.

- Check if oil tank and oil pipelines heated by steam or electricity are insulated.

- Identify how much condensate is returned and if it is metered. If not try to estimate by other means.

- Check if blow down practice is adequate and blow down valve is not leaking.

- Check if flue gas dampers are installed in stand by boilers to avoid heat losses.

- Check the possibility to retrofit boilers with economizer if not equipped already.

- Check steam distribution system for proper insulation of pipelines, valves and fittings.

- Conduct leakage test for steam traps, valves and fittings.

- Check if housekeeping is adequately done.

- Develop mass and energy balance of boilers in factory.

\subsubsection{Motors}

- Motors are not all the same. Be careful not to think that what is good for one is good for all. For example, some motors require a periodic greasing of the bearings and some do not.

- Facility managers should inventory all motors in their facilities, beginning with the 
largest and those with the longest run-times. This inventory enables facility managers to make informed choices about replacement either before or after motor failure. Field testing motors prior to failure enables the facility manager to properly size replacements to match the actual driven load.

- Clean motor surfaces and ventilation openings periodically, preferably with a vacuum cleaner, heavy accumulations of dust and lint will result in overheating and premature motor failure.

- Turn off or sequence unnecessary motors.

- Check packing for wear and repack as necessary. Consider replacing packing with mechanical seals.

- Check and secure all motor mounting.

- Tighten connection terminals as necessary.

- Inspect bearings and drive belts for wear.

- Check the condition for the motor through temperature or vibration analysis to assure long life.

- Unbalanced power can shorten the motor life through excessive heat buildup.

- Over- or under-voltage situations can shorten the motor life through excessive heat buildup.

\subsubsection{HVAC}

- Monitor outside air use.

- Avoid heating and cooling at the same time. 
- Use modular, localized heating/cooling units where possible.

- Control system by time-of-use when possible.

- Shut off chiller during winter if possible.

- Seal ductwork leaks.

- Clean filters and allow free air-flow to grills.

- Seal unused building openings.

- Install vinyl curtains in loading areas.

- Weather-strip doors and windows, caulk cracks.

- Insulate doors, pipes, and ductwork.

- Use ceiling fans to increase comfort.

- Clean boilers, chillers and condenser coils regularly.

- Do not heat/cool in low traffic areas, hallways or unoccupied areas.

- Adjust building temperature by season.

- Utilize available passive solar heat during cooler months by opening blinds and drapes.

- Close doors to outside and unheated or uncooled areas.

- Use ventilation only when required.

- Establish routine maintenance procedures.

\subsubsection{Furnaces and Ovens}

- Check the air/fuel ratio and keep it at an optimum level.

- Check insulation for the furnace to reduce the heat loss. 
- Clean the chamber, and remove all soots and particles inside the chamber to prevent the incomplete combustion and explosion.

- Check the chamber to minimize the air infiltration in the furnaces.

- Check the door or open area of the chamber and seek for a possibility to minimize the size of the opening area.

- Check all burner heads in a good working condition to prevent loss injecting the fuel.

\subsubsection{Production Improvement}

Improving any of the followings could be possibility to improve the operation and hence to reduce energy usage:

- Quality of products and services

- Production rate

- Delivery time and method to customer

- Inspection and testing

- Corrective and preventive action

- Process control

- Supplier qualification

- Diversity of products and processes in an assembly line

- Manufacturing bottlenecks

- Labor allocation

- Safety awareness 
- Manufacturing strategic plan (Policies, developing strategies, simulation of the strategies)

\subsection{Evaluation and Continuous Improvement}

The EEKC needs to be continuously evaluated to find any possible bugs or improvement suggestions. The Plan-Do-Check-Adjust (PDCA) is used for continuous improvement of the EEKC.

PDCA is known as the model for continuous improvement. The four-phase approach provides a simplified sequence to carrying out change [18]. Figure 34 shows the evaluation and continuous improvement steps. Not only the system should be evaluated by the IAC directors and lead students but user collaboration with the system needs to be also evaluated. Then, based on needs identified, the PDCA could be used to make improvement. 


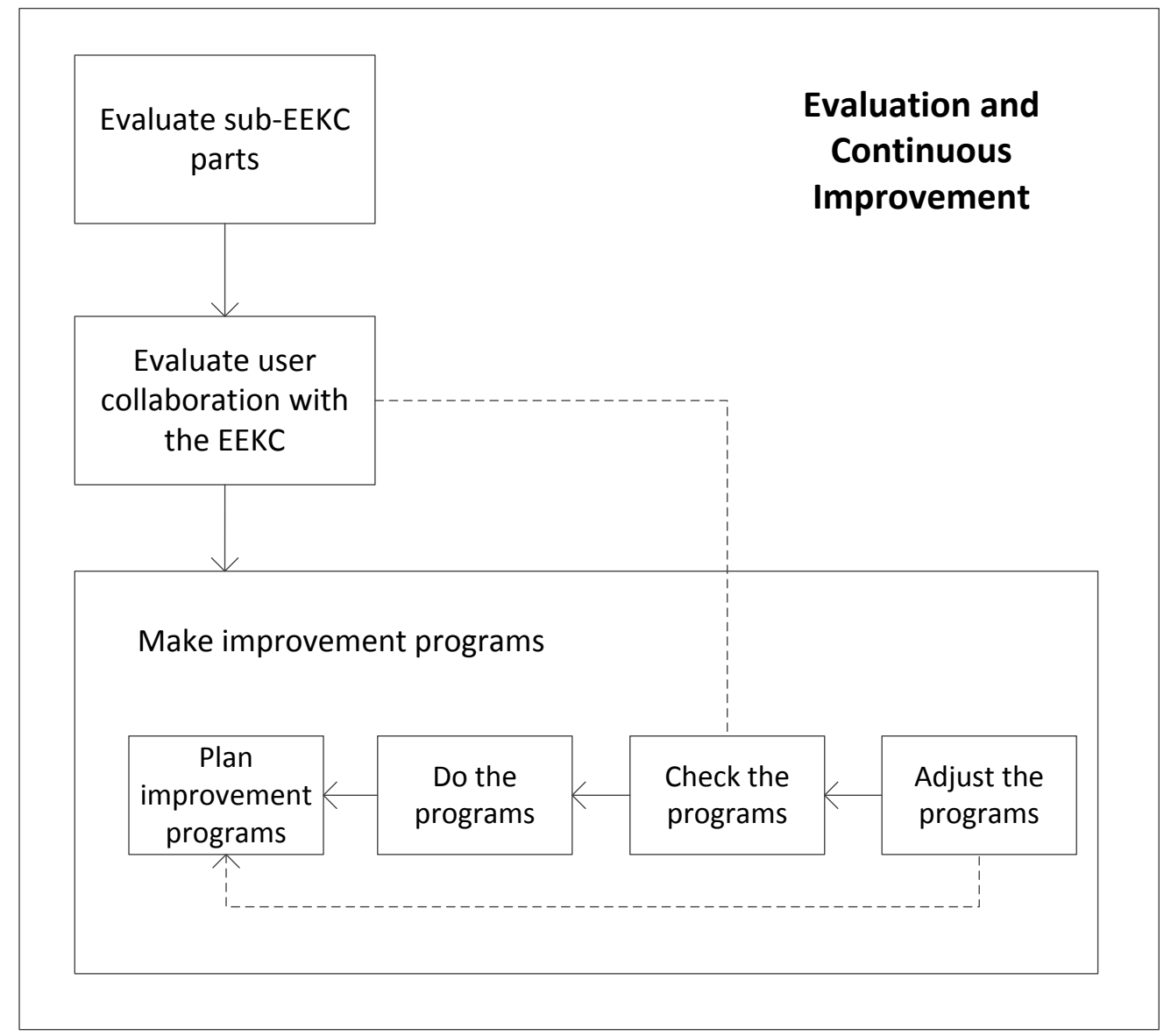

Figure 34: Evaluation and improvement of the EEKC 


\section{CHAPTER 5: DISCUSSIONANDCONCLUSION}

Energy management is very essential for companies to be competitive in global business markets. They must extract the highest possible percentage of productivity from each BTU of energy. The IAC program helps manufacturing facilities cut back on unnecessary costs from inefficient energy use, ineffective production procedures, excess waste production, and other production-related problems [27]. Continuous improvement in work processes of the energy efficiency assessment reports is one of the main missions of the IAC program. It was expected that managing information and knowledge within the IAC enhances the continuous improvement process. To manage the information and knowledge, the EEKC was designed in which information and knowledge can be securely saved, validated, and shared.

This research study focused on the SEN assessment activities and processes, and then, based on needs identified, the EEKC was developed. To ensure that the EEKC works in accordance with the IAC needs, three usage protocols were also developed. The protocols were followed up for a specific assessment and then the applications required in the EEKC were developed.

The EEKC helps the IAC not only reduce the report development time by preparing the necessary applications and information and knowledge validated, but also it helps to increase the productivity and report quality. The EEKC needs to be continuously evaluated and improved. It is also critical that the usage protocols are followed by the IAC members. Providing necessary references in a unique and user friendly site along with the ability to write and edit the content make it a suitable tool to be used in IAC 
work processes. IAC directors can also ask the IAC students to use the system and encourage them by the usage advantages.

In addition to the IAC members, clients can also use the EEKC and view their assessment results in the system. If they are given permission, they would be able to navigate into knowledge center and other private parts of the EEKC. DOE personnel can also navigate into the entire system and monitor it.

If the EEKC is used in all SEN assessments, it would receive more and more entries over time and becomes a valuable resource. To ensure that this significant bank is properly protected and secured, high security services of Google are used.

\section{Future Work}

By increasing the number of reports entered into the EEKC, the huge amount of data and information will be saved in the system in which knowledge extracted in Wiki could be still as short as possible; but if an advanced tool i.e. text mining used to analyze the data and text in each AR, the relationships between different ARs and their effects on each other could be automatically identified. This may significantly help the IAC program enhance the energy efficiency reports and improve the amount of savings. 


\section{$\underline{\text { R E F E R E N C E S }}$}

[1] Industrial Assessment Center, West Virginia University, obtained in May 2011 from: http://www2.cemr.wvu.edu/ wwwiac/overview.html

[2] Best Practices, Industrial Assessment Centers (IACs), Department of Energy, obtained in May 2011 from: http://www1.eere.energy.gov/industry/bestpractices/iacs.html

[3] Milton, N.R., 2007. Knowledge Acquisition in Practice: A Step-by-step Guide (Decision Engineering), Springer, London.

[4] Nonaka, Takeuchi, 1995. The Knowledge Creating Company, Oxford University Press, New York.

[5] Koskinen, K.U., Pihlanto, P., Vanharanta, H., 2003. Tacit knowledge acquisition and sharing in a project work context, International Journal of Project Management, 21: 281290.

[6] Montana, J.C., 2000. The legal system and knowledge management, The Information Management Journal, 54-57.

[7] Lai, I.L.A., 2005. Knowledge management for Chinese medicines: a conceptual model, Information Management \& Computer Security, 13(3): 244-255.

[8] Scholl, W., König, C., Meyer, B., Heisig, P., 2004. The future of knowledge management: an International Delphi Study, Journal of knowledge Management 8(2): 1935.

[9] Bali, R.K., 2005. Clinical knowledge management: opportunities and challenges, IDEA Group, 120-121.

[10] Turban, E., Aronson, J.E., Liang, T-P., Sharda, R., 2006. Decision Support and Business Intelligence Systems, 8th edition, Prentice Hall. 
[11] Tripathi, M., Kumar, S., 2010. Use of Web 2.0 tools in academic libraries: A reconnaissance of the international landscape, The International Information \& Library Review, 42: 195-207.

[12] Moria Levy, 2009. WEB 2.0 implications on knowledge management, Journal of Knowledge Management, 13(1): 120-134.

[13] Wiki, obtained in May 2011 from: http://en.wikipedia.org/wiki/Wiki

[14] Michel B., Fabien G., Guillaume E., Peter S., Catherine F., 2008. SweetWiki: A semantic Wiki, Web Semantics: Science, Services and Agents, 6: 84-97.

[15] Quick Plant Energy Profiler, Department of Energy, obtained in May 2011 from: http://www1.eere.energy.gov/industry/quickpep_ml/

[16] Industrial Assessment Centers Database, Department of Energy, obtained in May 2011 from: http://iac.rutgers.edu/database/

[17] Governance model for SharePoint Server 2010, Microsoft, obtained in May 2011 from: http://www.microsoft.com/downloads/

[18] Continuous Improvement, Michigan Tech, obtained in Jun 2011 from:

http://www.mtu.edu/improvement/follow-up/pdca/

[19] IAC, University of Florida, Obtained in Jul 2011 from:

http://www.ise.ufl.edu/iac/assessments.htm

[20] IAC, University of Missouri-Columbia, Obtained in Jul 2011 from:

http://iac.missouri.edu/webtool/TaskDocuments/

[21] HTTP Secure, Wikipedia, Obtained in Jul 2011 from:

http://en.wikipedia.org/wiki/HTTP_Secure

[22] Völkera L., Noea M., Waldhorsta O. P., Werlea C. and Sorgeb C., 2011. Can internet users protect themselves? Challenges and techniques of automated protection of HTTP communication, Computer Communications, 34(3): 457-467. 
[23] What is the Difference Between http and https?, WiseGEEK, Obtained in Jul 2011 from: http://www.wisegeek.com/what-is-the-difference-between-http-and-https.htm

[24] Google firms up Gmail security, 2008. Network Security, 2008(9): 2.

[25] Google Sites Help, Obtained in Jul 2011 from:

http://sites.google.com/support/bin/answer.py?answer=90594

[26] Google Sites Help, Obtained in Jul 2011 from:

http://www.google.com/support/pages/bin/answer.py?hl=en\&answer=90597

[27] Industrial Assessment Center, Wikipedia, Obtained in Jul 2011 from:

http://en.wikipedia.org/wiki/Industrial_Assessment_Center

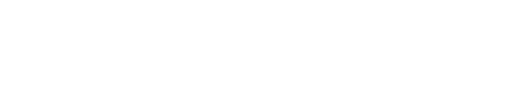

ou=Acquisitions Department,

Hagen 